\title{
STOCHASTIC REPRESENTATION OF SOLUTIONS TO DEGENERATE ELLIPTIC AND PARABOLIC BOUNDARY VALUE AND OBSTACLE PROBLEMS WITH DIRICHLET BOUNDARY CONDITIONS
}

\author{
PAUL M. N. FEEHAN AND CAMELIA A. POP
}

\begin{abstract}
We prove existence and uniqueness of stochastic representations for solutions to elliptic and parabolic boundary value and obstacle problems associated with a degenerate Markov diffusion process. In particular, our article focuses on the Heston stochastic volatility process, which is widely used as an asset price model in mathematical finance and a paradigm for a degenerate diffusion process where the degeneracy in the diffusion coefficient is proportional to the square root of the distance to the boundary of the half-plane. The generator of this process with killing, called the elliptic Heston operator, is a second-order, degenerate, elliptic partial differential operator whose coefficients have linear growth in the spatial variables and where the degeneracy in the operator symbol is proportional to the distance to the boundary of the half-plane. In mathematical finance, solutions to terminal/boundary value or obstacle problems for the parabolic Heston operator correspond to value functions for American-style options on the underlying asset.
\end{abstract}

\section{Contents}

1. Introduction

2. Properties of the Heston stochastic volatility process

3. Elliptic boundary value problem

4. Elliptic obstacle problem

5. Parabolic terminal/boundary value problem

6. Parabolic obstacle problem

Appendix A. Local a priori boundary estimates

Appendix B. Regular points and continuity properties of stochastic representations

Acknowledgments

References

Received by the editors September 24, 2012 and, in revised form, December 3, 2012. 2010 Mathematics Subject Classification. Primary 60J60; Secondary 35J70, 35R45.

Key words and phrases. Degenerate elliptic and parabolic differential operators, degenerate diffusion process, Feller square root process, Feynman-Kac formula, Heston stochastic volatility process, mathematical finance, degenerate stochastic differential equation, stochastic representation formula.

The first author was partially supported by NSF grant DMS-1059206. The second author was partially supported by a Rutgers University fellowship. 


\section{INTRODUCTION}

Since its discovery by Mark Kac [29], inspired in turn by the doctoral dissertation of Richard Feynman [19], the Feynman-Kac (or stochastic representation) formula has provided a link between probability theory and partial differential equations which has steadily deepened and developed during the intervening years. Moreover, judging by continuing interest in its applications to mathematical finance 31] and mathematical physics [37, 41, including non-linear parabolic equations [7, this trend shows no sign of abating. However, while stochastic representation formulae for solutions to linear, second-order elliptic and parabolic boundary and obstacle problems are well established when the generator, $-A$, of the Markov stochastic process is strictly elliptic [5, 24,30,38 in the sense of [25, p. 31], the literature is far less complete when $A$ is degenerate elliptic, that is, only has a non-negative definite characteristic form in the sense of [39], and its coefficients are unbounded.

In this article, we prove stochastic representation formulae for solutions to an elliptic boundary value problem,

$$
A u=f \quad \text { on } \mathscr{O},
$$

and an elliptic obstacle problem,

$$
\min \{A u-f, u-\psi\}=0 \quad \text { on } \mathscr{O},
$$

respectively, subject to a partial Dirichlet boundary condition,

$$
u=g \quad \text { on } \Gamma_{1} .
$$

Here, the subset $\mathscr{O} \subseteq \mathbb{H}$ is a (possibly unbounded) domain (connected, open subset) in the open upper half-space $\mathbb{H}:=\mathbb{R}^{d-1} \times(0, \infty)$ (where $\left.d \geq 2\right), \Gamma_{1}=\partial \mathscr{O} \cap \mathbb{H}$ is the portion of the boundary, $\partial \mathscr{O}$, of $\mathscr{O}$ which lies in $\mathbb{H}, f: \mathscr{O} \rightarrow \mathbb{R}$ is a source function, the function $g: \Gamma_{1} \rightarrow \mathbb{R}$ prescribes a Dirichlet boundary condition along $\Gamma_{1}$ and $\psi: \mathscr{O} \cup \Gamma_{1} \rightarrow \mathbb{R}$ is an obstacle function which is compatible with $g$ in the sense that

$$
\psi \leq g \text { on } \Gamma_{1},
$$

while $A$ is an elliptic differential operator on $\mathscr{O}$ which is degenerate along the interior, $\Gamma_{0}$, of $\partial \mathbb{H} \cap \partial \mathscr{O}$ and may have unbounded coefficients. We require $\Gamma_{0}$ to be non-empty throughout this article as, otherwise, if $\mathscr{O}$ is bounded (and the coefficients of $A$ are, say, continuous on $\overline{\mathscr{O}})$, then standard results apply [5, 24, 30, 38, However, an additional boundary condition is not necessarily prescribed along $\Gamma_{0}$. Rather, we shall see that our stochastic representation formulae will provide the unique solutions to (1.1) or (1.2), together with (1.3), when we seek solutions which are suitably smooth up to the boundary portion $\Gamma_{0}$, a property which is guaranteed when the solutions lie in certain weighted Hölder spaces (by analogy with [1] ), or replace the boundary condition (1.3) with the full Dirichlet condition,

$$
u=g \text { on } \partial \mathscr{O} \text {, }
$$

in which case the solutions are not guaranteed to be any more than continuous up to $\Gamma_{0}$ and $\psi: \overline{\mathscr{O}} \rightarrow \mathbb{R}$ is now required to be compatible with $g$ in the sense that

$$
\psi \leq g \text { on } \partial \mathscr{O} \text {. }
$$

We also prove stochastic representation formulae for solutions to a parabolic terminal/boundary value problem,

$$
-u_{t}+A u=f \quad \text { on } Q,
$$


and a parabolic obstacle problem,

$$
\min \left\{-u_{t}+A u-f, u-\psi\right\}=0 \quad \text { on } Q,
$$

respectively, subject to the partial terminal/boundary condition,

$$
u=g \quad \text { on } \partial^{1} Q .
$$

Here, we define $Q:=(0, T) \times \mathscr{O}$, where $0<T<\infty$, and define

$$
\check{\partial}^{1} Q:=(0, T) \times \Gamma_{1} \cup\{T\} \times\left(\mathscr{O} \cup \Gamma_{1}\right)
$$

to be a subset of the parabolic boundary of $Q$, and we assume given a source function $f: Q \rightarrow \mathbb{R}$, a Dirichlet boundary data function $g: \partial^{1} Q \rightarrow \mathbb{R}$, and an obstacle function $\psi: Q \cup \partial^{1} Q \rightarrow \mathbb{R}$ which is compatible with $g$ in the sense that,

$$
\psi \leq g \text { on } \check{\partial}^{1} Q \text {. }
$$

Just as in the elliptic case, we shall either consider solutions which are suitably smooth up to $(0, T) \times \Gamma_{0}$, but impose no explicit Dirichlet boundary condition along $(0, T) \times \Gamma_{0}$, or replace the boundary condition in (1.9) with the full Dirichlet condition

$$
u=g \quad \text { on } \check{\partial},
$$

where

$$
\check{\partial}:=(0, T) \times \partial \mathscr{O} \cup\{T\} \times \overline{\mathscr{O}}
$$

is the full parabolic boundary of $Q$, in which case the solutions are not guaranteed to be any more than continuous up to $(0, T) \times \Gamma_{0}$ and $\psi: Q \cup ð Q \rightarrow \mathbb{R}$ is now compatible with $g$ in the sense that

$$
\psi \leq g \quad \text { on } \precsim Q
$$

Before giving a detailed account of our main results, we summarize a few applications.

1.1. Applications. In mathematical finance, a solution, $u$, to the elliptic obstacle problem (1.2), (1.3), when $f=0$, can be interpreted as the value function for a perpetual American-style option with payoff function given by the obstacle function, $\psi$, while a solution, $u$, to the corresponding parabolic obstacle problem (1.8), (1.9), when $f=0$, can be interpreted as the value function for a finite-maturity Americanstyle option with payoff function given by a terminal condition function, $h=$ $g(T, \cdot): \mathscr{O} \rightarrow \mathbb{R}$, which typically coincides on $\{T\} \times \mathscr{O}$ with the obstacle function, $\psi$. For example, in the case of an American-style put option, one chooses $\psi(x, y)=$ $\left(E-e^{x}\right)^{+}$, for all $(x, y) \in \mathscr{O}$, where $E>0$ is a positive constant. While solutions to (1.1), (1.3) do not have an immediate interpretation in mathematical finance, a solution, $u$, to the corresponding parabolic terminal/boundary value problem (1.7), (1.9), when $f=0$, can be interpreted as the value function for a European-style option with payoff function given by the terminal condition function, $h$. For example, in the case of a European-style put option, one chooses $h(x, y)=\left(E-e^{x}\right)^{+}$, for all $(x, y) \in \mathscr{O}$.

Stochastic representation formulae underly Monte Carlo methods of numerical computation of value functions for option pricing in mathematical finance [26]. As is well known to practitioners, the question of Monte Carlo simulation of solutions to the Heston stochastic differential equation is especially delicate [3, 36. We hope that our article sheds further light on these issues. 
1.2. Summary of main results. In this article, we set $d=2$ and choose $-A$ to be the generator of the two-dimensional Heston stochastic volatility process with killing rate $r$ [27, a degenerate diffusion process well known in mathematical finance:

(1.15)

$-A v:=\frac{y}{2}\left(v_{x x}+2 \rho \sigma v_{x y}+\sigma^{2} v_{y y}\right)+(r-q-y / 2) v_{x}+\kappa(\theta-y) v_{y}-r v, \quad v \in C^{\infty}(\mathbb{H})$.

Nonetheless, we expect that many of our results would extend to a much broader class of degenerate Markov processes, and we shall address such questions elsewhere. Throughout this article, the coefficients of $A$ are required to obey

Assumption 1.1 (Ellipticity condition for the Heston operator coefficients). The coefficients defining $A$ in (1.15) are constants obeying

$$
\sigma \neq 0, \quad-1<\rho<1,
$$

and $\kappa>0, \theta>0$, and $q, r \in \mathbb{R}$.

Let $(\Omega, \mathscr{F}, \mathbb{F}, \mathbb{Q})$ be a filtered probability space satisfying the usual conditions, where $\mathbb{F}=\{\mathscr{F}(s)\}_{s \geq 0}$ is the $\mathbb{Q}$-completion of the natural filtration of $(W(s))_{s \geq 0}$, and $(W(s))_{s>0}$ is a standard Brownian motion with values in $\mathbb{R}^{2}$. For $0 \leq t<$ $T<\infty$, let $\mathscr{T}_{t, T}$ denote the set of $\mathbb{F}$-stopping times with values in $[t, T]$. Let $\left(X^{t, x, y}(s), Y^{t, y}(s)\right)_{s \geq t}$ denote a continuous version of the strong solution to the Heston stochastic differential equation,

$$
\begin{aligned}
d X(s)= & \left(r-q-\frac{Y(s)}{2}\right) d s+\sqrt{Y(s)} d W_{1}(s), \\
d Y(s)= & \kappa(\vartheta-Y(s)) d s+\sigma \sqrt{Y(s)}\left(\rho d W_{1}(s)+\sqrt{1-\rho^{2}} d W_{2}(s)\right), \\
& (X(t), Y(t))=(x, y),
\end{aligned}
$$

where $s>t$, which exists by Corollary 2.8, where the coefficients are as in Assumption 1.1. For brevity, we sometimes denote $z=(x, y)$ and $\left(Z^{t, z}(s)\right)_{s>t}=$ $\left(X^{t, x, y}(s), Y^{t, y}(s)\right)_{s \geq t}$. We omit the superscripts $(t, z)$ and $(t, x, y)$ when the initial condition is clear from the context, or we omit the superscript $t$ when $t=0$. We let

$$
\begin{aligned}
\beta & :=\frac{2 \kappa \vartheta}{\sigma^{2}}, \\
\mu & :=\frac{2 \kappa}{\sigma^{2}}
\end{aligned}
$$

denote the Feller parameters associated with the Heston process.

1.2.1. Existence and uniqueness of solutions to elliptic boundary value problems. For an integer $k \geq 0$, we let $C^{k}(\mathscr{O})$ denote the vector space of functions whose derivatives up to order $k$ are continuous on $\mathscr{O}$ and let $C^{k}(\overline{\mathscr{O}})$ denote the Banach space of functions whose derivatives up to order $k$ are uniformly continuous and bounded on $\mathscr{O}[2, \S 1.25 \& \S 1.26]$. If $T \varsubsetneqq \partial \mathscr{O}$ is a relatively open set, we let $C_{\text {loc }}^{k}(\mathscr{O} \cup T)$ denote the vector space of functions, $u$, such that, for any precompact open subset $U \Subset \mathscr{O} \cup T$, we have $u \in C^{k}(\bar{U})$.

\footnotetext{
${ }^{1}$ We impose additional conditions, such as $q \geq 0, r \geq 0$, or $r>0$, depending on the problem under consideration. We only require that $q \geq 0$ when deriving the supermartingale property in Lemma 2.11 (1), a property used only in the elliptic case.
} 
We shall often appeal to the following

Hypothesis 1.2 (Growth condition). If $v$ is a function, then for all $(x, y)$ in its domain of definition,

$$
|v(x, y)| \leq C\left(1+e^{M_{1} y}+e^{M_{2} x}\right),
$$

where $C>0,0 \leq M_{1}<\min \{r /(\kappa \vartheta), \mu\}$, and $M_{2} \in[0,1)$.

Let $U \subseteq \mathbb{H}$ be an open set. We denote

$$
\tau_{U}^{t, z}:=\inf \left\{s \geq t: Z^{t, z}(s) \notin U\right\},
$$

and we let

$$
\nu_{U}^{t, z}:=\inf \left\{s \geq t: Z^{t, z}(s) \notin U \cup(\bar{U} \cap \partial \mathbb{H})\right\} .
$$

Notice that if $\bar{U} \cap \partial \mathbb{H}=\varnothing$, then $\tau_{U}^{t, z}=\nu_{U}^{t, z}$. We also have that $\tau_{U}^{t, z}=\nu_{U}^{t, z}$ when $\beta \geq 1$, because in this case the process $Z^{t, z}$ does not reach the boundary $\partial \mathbb{H}$, by Lemma 2.10 (1). By [38, p. 117], both $\tau_{U}^{t, z}$ and $\nu_{U}^{t, z}$ are stopping times with respect to the filtration $\mathbb{F}$, since $\mathbb{F}$ is assumed to satisfy the usual conditions. When the initial condition, $(t, z)$, is clear from the context, we omit the superscripts in the preceding definitions (1.21) and (1.22) of the stopping times. Also, when $t=0$, we omit the superscript $t$ in the preceding definitions.

Theorem 1.3 (Uniqueness of solutions to the elliptic boundary value problem). Let $r>0, q \geq 0$, and $f$ be a Borel measurable function $2^{2}$ on $\mathscr{O}$ which obeys the growth condition (1.20) on $\mathscr{O}$. Then

(1) If $\beta \geq 1$, assume $g \in C_{\mathrm{loc}}\left(\Gamma_{1}\right)$ obeys (1.20). Let

$$
u \in C_{\mathrm{loc}}\left(\mathscr{O} \cup \Gamma_{1}\right) \cap C^{1,1}(\mathscr{O})
$$

be a solution to the elliptic boundary value problem (1.1), (1.3) and which obeys (1.20) on $\mathscr{O}$. Then, $u=u^{*}$ on $\mathscr{O} \cup \Gamma_{1}$, where

$$
u^{*}(z):=\mathbb{E}_{\mathbb{Q}}^{z}\left[e^{-r \tau_{\mathscr{O}}} g\left(Z\left(\tau_{\mathscr{O}}\right)\right) 1_{\left\{\tau_{\mathscr{O}}<\infty\right\}}\right]+\mathbb{E}_{\mathbb{Q}}^{z}\left[\int_{0}^{\tau_{\mathscr{O}}} e^{-r s} f(Z(s)) d s\right],
$$

and $\tau_{\mathscr{O}}$ is defined by (1.21), for all $z \in \mathscr{O} \cup \Gamma_{1}$.

(2) If $0<\beta<1$, assume $g \in C_{\mathrm{loc}}(\partial \mathscr{O})$ obeys (1.20) on $\partial \mathscr{O}$, and let $u \in$ $C_{\mathrm{loc}}(\overline{\mathscr{O}}) \cap C^{1,1}(\mathscr{O})$ be a solution to the elliptic boundary value problem (1.1), (1.5) and which obeys (1.20) on $\mathscr{O}$. Then, $u=u^{*}$ on $\overline{\mathscr{O}}$, where $u^{*}$ is given by (1.23).

Following [9, Definition 2.2], let $C_{s, \text { loc }}^{1,1}\left(\mathscr{O} \cup \Gamma_{0}\right)$ denote the subspace of $C^{1,1}(\mathscr{O})$ consisting of functions, $u$, such that, for any precompact open subset $U \Subset \mathscr{O} \cup \Gamma_{0}$,

$$
\sup _{(x, y) \in U}|u(x, y)|+|D u(x, y)|+\left|y D^{2} u(x, y)\right|<\infty,
$$

where $D u$ denotes the gradient and $D^{2} u$ the Hessian matrix of $u$.

\footnotetext{
${ }^{2}$ We require $f$ to be Borel measurable in order to ensure that expectations such as those in (1.23) are well defined.
} 
Remark 1.4 (On the definition of $C_{s, \text { loc }}^{1,1}\left(\mathscr{O} \cup \Gamma_{0}\right)$ ). The definition of the function space $C_{s, \text { loc }}^{1,1}\left(\mathscr{O} \cup \Gamma_{0}\right)$ can be relaxed in our article from that of [9, Definition 2.2]. It is enough to say that a function $u$ belongs to $C_{s, \text { loc }}^{1,1}\left(\mathscr{O} \cup \Gamma_{0}\right)$ if $u \in C^{1,1}(\mathscr{O})$ and (1.24) holds on any precompact open subset $U \Subset \mathscr{O} \cup \Gamma_{0}$.

Theorem 1.5 (Uniqueness of solutions to the elliptic boundary value problem (1.1), (1.3), when $0<\beta<1)$. Let $r>0, q \geq 0,0<\beta<1$, and let $f \in C_{\text {loc }}\left(\mathscr{O} \cup \Gamma_{0}\right)$ be such that it satisfies condition (1.20). Let $g \in C_{\mathrm{loc}}\left(\Gamma_{1}\right)$ obey (1.20) on $\Gamma_{1}$ and suppose that

$$
u \in C_{\mathrm{loc}}\left(\mathscr{O} \cup \Gamma_{1}\right) \cap C_{s, \text { loc }}^{1,1}\left(\mathscr{O} \cup \Gamma_{0}\right)
$$

is a solution to the elliptic boundary value problem (1.1), (1.3) which obey (1.20) on $\mathscr{O}$. Then, $u=u^{*}$ on $\mathscr{O} \cup \Gamma_{1}$, where $u^{*}$ is given by

$$
u^{*}(z):=\mathbb{E}_{\mathbb{Q}}^{z}\left[e^{-r \nu_{\mathscr{O}}} g\left(Z\left(\nu_{\mathscr{O}}\right)\right) 1_{\left\{\nu_{\mathscr{O}}<\infty\right\}}\right]+\mathbb{E}_{\mathbb{Q}}^{z}\left[\int_{0}^{\nu_{\mathscr{O}}} e^{-r s} f(Z(s)) d s\right],
$$

and $\nu_{\mathscr{O}}$ is defined by (1.22), for all $z \in \mathscr{O} \cup \Gamma_{1}$.

Remark 1.6 (Existence and uniqueness of strong solutions in weighted Sobolev spaces to the elliptic boundary value problem). Existence and uniqueness of strong solutions in weighted Sobolev spaces to problem (1.1) with boundary condition (1.3) along $\Gamma_{1}$, for all $\beta>0$, is proved in [8, Theorem 1.18], and Hölder continuity of such solutions up to $\Gamma_{0}$ is proved in [15, Theorem 1.10].

Remark 1.7 (Comparison of uniqueness results). To obtain uniqueness of solutions to the elliptic boundary value problem (1.1) with boundary condition (1.3) only specified along $\Gamma_{1}$, we need to assume the stronger regularity hypothesis,

$$
u \in C_{\mathrm{loc}}\left(\mathscr{O} \cup \Gamma_{1}\right) \cap C_{s, \mathrm{loc}}^{1,1}\left(\mathscr{O} \cup \Gamma_{0}\right),
$$

when $0<\beta<1$, while the regularity assumption,

$$
u \in C_{\mathrm{loc}}\left(\mathscr{O} \cup \Gamma_{1}\right) \cap C^{1,1}(\mathscr{O}),
$$

suffices when $\beta \geq 1$. The analogous comments apply to the elliptic obstacle problems described in Theorems 1.12 and 1.13, the parabolic terminal/boundary value problems described in Theorems 1.16] and 1.18, and the parabolic obstacle value problems described in Theorems 1.27 and 1.28 .

For $\alpha \in(0,1)$, we let $C^{k+\alpha}(\mathscr{O})$ denote the subspace of $C^{k}(\mathscr{O})$ consisting of functions whose derivatives up to order $k$ are locally $\alpha$-Hölder continuous on $\mathscr{O}$ (in the sense of [25, p. 52]) and let $C^{k+\alpha}(\overline{\mathscr{O}})$ denote the subspace of $C^{k}(\overline{\mathscr{O}})$ consisting of functions whose derivatives up to order $k$ are uniformly $\alpha$-Hölder continuous on $\mathscr{O}$ [25, p. 52], [2, §1.27]. If $T \varsubsetneqq \partial \mathscr{O}$ is a relatively open set, we let $C_{\text {loc }}^{k+\alpha}(\mathscr{O} \cup T)$ denote the vector space of functions, $u$, such that, for any precompact open subset $U \Subset \mathscr{O} \cup T$, we have $u \in C^{k+\alpha}(\bar{U})$.

We have the following result concerning existence of solutions to the elliptic boundary value problem with traditional Hölder regularity on $\mathscr{O}$. 
Theorem 1.8 (Existence of solutions to the elliptic boundary value problem with continuous Dirichlet boundary condition). In addition to the hypotheses of Theorem 1.3, assume that the domain $\mathscr{O} \subset \mathbb{H}$ has boundary portion $\Gamma_{1}$ which satisfies the exterior sphere condition and that $f \in C^{\alpha}(\mathscr{O})$.

(1) If $\beta \geq 1$ and also $g \in C_{\mathrm{loc}}\left(\bar{\Gamma}_{1}\right)$, then the function $u^{*}$ in (1.23) is a solution to problem (1.1) with boundary condition (1.3) along $\Gamma_{1}$. In particular, $u^{*} \in C_{\mathrm{loc}}\left(\mathscr{O} \cup \Gamma_{1}\right) \cap C^{2+\alpha}(\mathscr{O})$ and $u^{*}$ satisfies the growth assumption (1.20).

(2) If $0<\beta<1$ and also $g \in C_{\mathrm{loc}}(\partial \mathscr{O})$, then the function $u^{*}$ in (1.23) is a solution to problem (1.1) with boundary condition (1.5) along $\partial \mathscr{O}$. In particular, $u^{*} \in C_{\mathrm{loc}}(\overline{\mathscr{O}}) \cap C^{2+\alpha}(\mathscr{O})$ and $u^{*}$ satisfies the growth assumption (1.20).

Theorem 1.9 (Existence of solutions to the elliptic boundary value problem with $C^{2+\alpha}$ continuous Dirichlet boundary condition). In addition to the hypotheses of Theorem 1.3, let $\mathscr{O} \subset \mathbb{H}$ be a domain such that the boundary portion $\Gamma_{1}$ is of class $C^{2+\alpha}$ and that $f \in C_{\mathrm{loc}}^{\alpha}\left(\mathscr{O} \cup \Gamma_{1}\right)$ and $g \in C_{\mathrm{loc}}^{2+\alpha}\left(\mathscr{O} \cup \Gamma_{1}\right)$.

(1) If $\beta \geq 1$, then $u^{*}$, given by (1.23), is a solution to problem (1.1) with boundary condition (1.3) along $\Gamma_{1}$. In particular,

$$
u^{*} \in C_{\mathrm{loc}}^{2+\alpha}\left(\mathscr{O} \cup \Gamma_{1}\right)
$$

and satisfies the growth assumption (1.20).

(2) If $0<\beta<1$ and $g \in C_{\mathrm{loc}}(\partial \mathscr{O})$, then $u^{*}$, given by (1.23), is a solution to problem (1.1) with boundary condition (1.5) along $\partial \mathscr{O}$. In particular,

$$
u^{*} \in C_{\mathrm{loc}}(\overline{\mathscr{O}}) \cap C^{2+\alpha}\left(\mathscr{O} \cup \Gamma_{1}\right)
$$

and satisfies the growth assumption (1.20).

Remark 1.10 (Existence of solutions with Daskalopoulos-Hamilton-Koch Hölder regularity). When $f \in C_{s}^{\alpha}\left(\mathscr{O} \cup \Gamma_{0}\right)$ and $g=0$, we establish in 17, Theorem 1.11 \& Corollary 1.13] that the solutions to the elliptic boundary value problem (1.1), (1.3) lie in $C_{s}^{2+\alpha}\left(\mathscr{O} \cup \Gamma_{0}\right) \cap C_{\text {loc }}(\overline{\mathscr{O}})$ for all $\beta>0$, where $C_{s}^{2+\alpha}\left(\mathscr{O} \cup \Gamma_{0}\right)$ is an elliptic analogue of the parabolic Daskalopoulos-Hamilton-Koch Hölder spaces described in [11,33]. A function $u \in C_{s}^{2+\alpha}\left(\mathscr{O} \cup \Gamma_{0}\right)$ has the property that $u, D u, y D^{2} u$ are $C_{s}^{\alpha}$ continuous up to $\Gamma_{0}$ and $y D^{2} u=0$ on $\Gamma_{0}$, where $C_{s}^{\alpha}\left(\mathscr{O} \cup \Gamma_{0}\right)$ is defined by analogy with the traditional definition of $C^{\alpha}(\mathscr{O})$ in $[25$, except that the Euclidean distance between points in $\mathscr{O}$ is replaced by the cycloidal distance function.

Remark 1.11 (Existence and uniqueness of solutions to elliptic boundary value problems). Existence and uniqueness of solutions to the elliptic boundary value problem (1.1) and (1.3), provided $\Gamma_{1}=\partial \mathscr{O}$, follow from Schauder methods when the coefficient matrix, $\left(a^{i j}\right)$, of the second-order derivatives in $A$ is uniformly elliptic. For example, see [25, Theorem 6.13] for the case where $\mathscr{O}$ is bounded and where $f$ and the coefficients of $A$ are bounded and in $C^{\alpha}(\mathscr{O}), \alpha \in(0,1)$, giving a unique solution $u \in C^{2+\alpha}(\mathscr{O}) \cap C(\overline{\mathscr{O}})$, while [25, Theorem 6.14] gives $u \in C^{2+\alpha}(\overline{\mathscr{O}})$ when $f$ and the coefficients of $A$ are in $C^{\alpha}(\overline{\mathscr{O}})$. See [34, Corollary 7.4.4], together with [34, Corollary 7.4.9] or [34, Theorem 7.6.4] or [34, Theorem 7.6.5 \& Remark 7.6.6], for similar statements. 
1.2.2. Uniqueness of solutions to elliptic obstacle problems. For $\theta_{1}, \theta_{2} \in \mathscr{T}$, we set

$$
\begin{aligned}
J_{e}^{\theta_{1}, \theta_{2}}(z):= & \mathbb{E}_{\mathbb{Q}}^{z}\left[\int_{0}^{\theta_{1} \wedge \theta_{2}} e^{-r s} f(Z(s)) d s\right] \\
& +\mathbb{E}_{\mathbb{Q}}^{z}\left[e^{-r \theta_{1}} g\left(Z\left(\theta_{1}\right)\right) 1_{\left\{\theta_{1} \leq \theta_{2}\right\}}\right]+\mathbb{E}_{\mathbb{Q}}^{z}\left[e^{-r \theta_{2}} \psi\left(Z\left(\theta_{2}\right)\right) 1_{\left\{\theta_{2}<\theta_{1}\right\}}\right] .
\end{aligned}
$$

We then have

Theorem 1.12 (Uniqueness of solutions to the elliptic obstacle problem). Let $r>0, q \geq 0$, and $f$ be as in Theorem 1.3, and $\psi$ be a Borel measurable function satisfying (1.20) on $\mathscr{O}$.

(1) If $\beta \geq 1$, let $\psi \in C_{\mathrm{loc}}\left(\mathscr{O} \cup \Gamma_{1}\right)$ and $g \in C_{\mathrm{loc}}\left(\Gamma_{1}\right)$ obey (1.20) and (1.4) on $\Gamma_{1}$. Let

$$
u \in C_{\mathrm{loc}}\left(\mathscr{O} \cup \Gamma_{1}\right) \cap C^{1,1}(\mathscr{O})
$$

be a solution to the elliptic obstacle problem (1.2), (1.3) such that $u$ and Au obey (1.20) on $\mathscr{O}$. Then, $u=u^{*}$ on $\mathscr{O} \cup \Gamma_{1}$, where $u^{*}$ is given by

$$
u^{*}(z):=\sup _{\theta \in \mathscr{T}} J_{e}^{\tau_{\mathscr{O}}, \theta}(z)
$$

and $\tau_{\mathscr{O}}$ is defined by (1.21), for all $z \in \mathscr{O} \cup \Gamma_{1}$.

(2) If $0<\beta<1$, let $\psi \in C_{\mathrm{loc}}(\overline{\mathscr{O}})$ and $g \in C_{\mathrm{loc}}(\partial \mathscr{O})$ obey (1.20) and (1.6) on

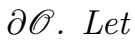

$$
u \in C_{\mathrm{loc}}(\overline{\mathscr{O}}) \cap C^{1,1}(\mathscr{O})
$$

be a solution to the elliptic obstacle problem (1.2), (1.5), such that $u$ and Au obey (1.20) on $\mathscr{O}$. Then, $u=u^{*}$ on $\overline{\mathscr{O}}$, where $u^{*}$ is given by (1.27).

Theorem 1.13 (Uniqueness of solutions to the elliptic obstacle problem (1.2), (1.3), when $0<\beta<1$ ). Let $r>0, q \geq 0,0<\beta<1$, and $f$ be as in Theorem 1.5. Let $\psi \in C_{\mathrm{loc}}(\overline{\mathscr{O}})$ obey (1.20) on $\mathscr{O}$ and let $g \in C_{\mathrm{loc}}\left(\Gamma_{1}\right)$ obey (1.20) and (1.4) on $\Gamma_{1}$. If

$$
u \in C_{\mathrm{loc}}(\overline{\mathscr{O}}) \cap C_{s, \mathrm{loc}}^{1,1}\left(\mathscr{O} \cup \Gamma_{0}\right)
$$

is a solution to the elliptic obstacle problem (1.2), (1.3) such that $u$ and Au obey (1.20), then $u=u^{*}$ on $\mathscr{O} \cup \Gamma_{1}$, where $u^{*}$ is given by

$$
u^{*}(z):=\sup _{\theta \in \mathscr{T}} J_{e}^{\nu_{\mathscr{O}}, \theta}(z)
$$

and $\nu_{\mathscr{O}}$ is defined by (1.22), for all $z \in \mathscr{O} \cup \Gamma_{1}$.

Remark 1.14 (Existence and uniqueness of strong solutions in weighted Sobolev spaces to the elliptic obstacle problem). Existence and uniqueness of strong solutions in weighted Sobolev spaces to problem (1.2) with Dirichlet boundary condition (1.3) along $\Gamma_{1}$, for all $\beta>0$, is proved in [8, Theorem 1.6], and Hölder continuity of such solutions up to the boundary portion $\Gamma_{0}$ is proved in [15, Theorem 1.13].

1.2.3. Existence and uniqueness of solutions to parabolic terminal/boundary value problems. We shall need to appeal to the following analogue of Hypothesis 1.2.

Hypothesis 1.15 (Growth condition). If $v$ is a function, then for all $(t, x, y)$ in its domain of definition,

$$
|v(t, x, y)| \leq C\left(1+e^{M_{1} y}+e^{M_{2} x}\right),
$$

where $C>0,0 \leq M_{1}<\mu$, and $M_{2} \in[0,1]$. 
For $Q=(0, T) \times \mathscr{O}$, we let $C(Q)$ denote the vector space of continuous functions on $Q$, while $C(\bar{Q})$ denotes the Banach space of functions which are uniformly continuous and bounded on $Q$. We let $D u$ denote the gradient and let $D^{2} u$ denote the Hessian matrix of a function $u$ on $Q$ with respect to spatial variables. We let $C^{1}(Q)$ denote the vector space of functions, $u$, such that $u, u_{t}$, and $D u$ are continuous on $Q$, while $C^{1}(\bar{Q})$ denotes the Banach space of functions, $u$, such that $u, u_{t}$, and $D u$ are uniformly continuous and bounded on $Q$. Finally, $C^{2}(Q)$ denotes the vector space of functions, $u$, such that $u_{t}, D u$, and $D^{2} u$ are continuous $Q$, while $C^{2}(\bar{Q})$ denotes the Banach space of functions, $u$, such that $u, u_{t}, D u$, and $D^{2} u$ are uniformly continuous and bounded on $Q$. If $T \varsubsetneqq \partial Q$ is a relatively open set, we let $C_{\text {loc }}(Q \cup T)$ denote the vector space of functions, $u$, such that, for any precompact open subset $V \Subset Q \cup T$, we have $u \in C(\bar{V})$.

Theorem 1.16 (Uniqueness of solutions to the parabolic terminal/boundary value problem). Let $f$ be a Borel measurable function on $Q$ and obey (1.29). Then

(1) If $\beta \geq 1$, assume $g \in C_{\mathrm{loc}}\left(\check{\partial}^{1} Q\right)$ obeys (1.29) on $\mathrm{\partial}^{1} Q$. Let

$$
u \in C_{\mathrm{loc}}\left(Q \cup \mathrm{\partial}^{1} Q\right) \cap C^{1,1}(Q)
$$

be a solution to the parabolic terminal/boundary value problem (1.7), (1.9) which obeys (1.29) on $Q$. Then, $u=u^{*}$ on $Q \cup \delta^{1} Q$, where $u^{*}$ is given by

$$
\begin{aligned}
u^{*}(t, z):= & \mathbb{E}_{\mathbb{Q}}^{t, z}\left[\int_{t}^{\tau_{\mathscr{O}} \wedge T} e^{-r(s-t)} f(s, Z(s)) d s\right] \\
& +\mathbb{E}_{\mathbb{Q}}^{t, z}\left[e^{-r\left(\tau_{\mathscr{O}} \wedge T-t\right)} g\left(\tau_{\mathscr{O}} \wedge T, Z\left(\tau_{\mathscr{O}} \wedge T\right)\right)\right],
\end{aligned}
$$

and $\tau_{\mathscr{O}}$ is defined by (1.21), for all $(t, z) \in Q \cup \mathrm{\partial}^{1} Q$.

(2) If $0<\beta<1$, assume $g \in C_{\mathrm{loc}}(ð Q)$ obeys (1.29) on $\precsim Q$, and let

$$
u \in C_{\mathrm{loc}}(Q \cup \partial Q) \cap C^{1,1}(Q)
$$

be a solution to the parabolic terminal/boundary value problem (1.7), (1.12) which obeys (1.29) on $Q$. Then, $u=u^{*}$ on $Q \cup ð Q$, where $u^{*}$ is given by (1.30).

Following [9, Definition 2.2], let $C_{s, \text { loc }}^{1,1}\left((0, T) \times\left(\mathscr{O} \cup \Gamma_{0}\right)\right)$ denote the subspace of $C^{1,1}((0, T) \times(\mathscr{O}))$ consisting of functions, $u$, such that, for any precompact open subset $V \Subset[0, T] \times\left(\mathscr{O} \cup \Gamma_{0}\right)$,

$$
\sup _{(t, z) \in V}|u(t, z)|+u_{t}(t, z)+|D u(t, z)|+\left|y D^{2} u(t, z)\right|<\infty .
$$

Remark 1.17 (On the definition of $C_{s, \text { loc }}^{1,1}\left((0, T) \times\left(\mathscr{O} \cup \Gamma_{0}\right)\right)$. The definition of the function space $C_{s, \text { loc }}^{1,1}\left((0, T) \times\left(\mathscr{O} \cup \Gamma_{0}\right)\right)$ can be relaxed in our article from that implied by [9, Definition 2.2]. It is enough to say that a function $u$ belongs to $C_{s, \text { loc }}^{1,1}\left((0, T) \times\left(\mathscr{O} \cup \Gamma_{0}\right)\right)$ if $u \in C^{1,1}(Q)$ and (1.31) holds on any precompact open subset $V \in(0, T) \times \Subset \mathscr{O} \cup \Gamma_{0}$.

We have the following alternative uniqueness result.

Theorem 1.18 (Uniqueness of solutions to the parabolic terminal/boundary value problems (1.7), (1.9), when $0<\beta<1)$. Let $0<\beta<1$ and $f \in C_{\mathrm{loc}}\left(Q \cup \partial^{1} Q\right)$ be such that it satisfies condition (1.29). Let $g \in C_{\mathrm{loc}}\left(\check{\partial}^{1} Q\right)$ obey (1.29) on $\check{\partial}^{1} Q$, and

$$
u \in C_{\mathrm{loc}}\left(Q \cup \check{\partial}^{1} Q\right) \cap C_{s, \text { loc }}^{1,1}\left((0, T) \times\left(\mathscr{O} \cup \Gamma_{0}\right)\right)
$$


be a solution to the parabolic terminal/boundary value problem (1.7), (1.9) which obeys (1.29) on $Q$. Then, $u=u^{*}$ on $Q \cup \varpi^{1} Q$, where $u^{*}$ is given by

$$
\begin{aligned}
u^{*}(t, z):= & \mathbb{E}_{\mathbb{Q}}^{t, z}\left[\int_{t}^{\nu_{\mathscr{O}} \wedge T} e^{-r(s-t)} f(s, Z(s)) d s\right] \\
& +\mathbb{E}_{\mathbb{Q}}^{t, z}\left[e^{-r\left(\nu_{\mathscr{O}} \wedge T-t\right)} g\left(\nu_{\mathscr{O}} \wedge T, Z\left(\nu_{\mathscr{O}} \wedge T\right)\right)\right],
\end{aligned}
$$

and $\nu_{\mathscr{O}}$ is defined by (1.22), for all $(t, z) \in Q \cup \check{\partial}^{1} Q$.

Remark 1.19 (Existence and uniqueness of strong solutions in weighted Sobolev spaces to the parabolic terminal/boundary value problem). Existence and uniqueness of strong solutions in weighted Sobolev spaces to problem (1.7) with Dirichlet boundary condition (1.9) along $\check{\partial}^{1} Q$, for all $\beta>0$, is proved in [10].

Remark 1.20 (Growth of solutions to parabolic boundary value problems). Karatzas and Shreve allow faster growth of solutions when the growth on the coefficients of the differential operator is constrained [30, Theorem 4.4.2 \& Problem 5.7.7], and polynomial growth of solutions is allowed for linear growth coefficients and source function $f$ with at most polynomial growth [30, Theorem 5.7.6].

Remark 1.21 (Barrier option pricing and discontinuous terminal/boundary conditions). In applications to finance, $\mathscr{O}$ will often be a rectangle, $\left(x_{0}, x_{1}\right) \times(0, \infty)$, where $-\infty \leq x_{0}<x_{1} \leq \infty$; the growth exponents will be $M_{1}=0$ and $M_{2}=1-$ indeed, the source function $f$ will always be zero and the spatial boundary condition function $g:(0, T) \times \Gamma_{1} \rightarrow \mathbb{R}$ will often be zero. However, the spatial boundary condition, $g:(0, T) \times \Gamma_{1} \rightarrow \mathbb{R}$, and terminal condition, $g:\{T\} \times \overline{\mathscr{O}} \rightarrow \mathbb{R}$, may be discontinuous where they meet along $\{T\} \times \partial \mathscr{O}$, as in the case of the down-and-out put, with

$$
g(t, x, y)= \begin{cases}0, & 0<t<T, x=x_{0}, y>0 \\ \left(K-e^{x}\right)^{+} & t=T, x_{0}<x<\infty, y>0\end{cases}
$$

where $g$ is discontinuous at $\left(T, x_{0}, y\right)$ if $K-e^{x_{0}}>0$, that is, $x_{0}<\log K$. We shall consider the question of establishing stochastic representations for solutions to parabolic terminal/value problems (European-style option prices) or parabolic obstacle problems (American-style option prices) with discontinuous data elsewhere.

For $\alpha \in(0,1)$, we let $C^{\alpha}(Q)$ denote the subspace of $C(Q)$ consisting of locally $\alpha$ Hölder continuous functions, $u$, on $Q$; that is, for any precompact open set $V \Subset Q$,

$$
[u]_{C^{\alpha}(V)}:=\sup _{\substack{\left(t^{i}, z^{i}\right) \in V \\ i=1,2}} \frac{\left|u\left(t^{1}, z^{1}\right)-u\left(t^{2}, z^{2}\right)\right|}{\left(\left|z^{1}-z^{2}\right|+\sqrt{\left|t^{1}-t^{2}\right|}\right)^{\alpha}}<\infty .
$$

Also, we let $C^{\alpha}(\bar{Q}) \subset C(\bar{Q})$ denote the Banach space of functions, $u$, which are uniformly $\alpha$-Hölder continuous on $Q$; that is

$$
[u]_{C^{\alpha}(Q)}<\infty .
$$

When $Q$ is unbounded, we let $C_{\mathrm{loc}}^{\alpha}(\bar{Q})$ denote the subspace of $C^{\alpha}(Q)$ consisting of functions, $u$, such that, for any precompact open set $V \Subset \bar{Q}$, we have

$$
[u]_{C^{\alpha}(V)}<\infty .
$$


We let $C^{2+\alpha}(Q)$ denote the subspace of $C^{2}(Q)$ consisting of functions, $u$, such that $u, u_{t}$, and the components of $D u$ and $D^{2} u$ belong to $C^{\alpha}(Q)$, and let $C^{2+\alpha}(\bar{Q}) \subset$ $C^{2}(\bar{Q})$ denote the Banach space of functions, $u$, such that $u, u_{t}$, and the components of $D u$ and $D^{2} u$ belong to $C^{\alpha}(\bar{Q})$.

Theorem 1.22 (Existence of solutions to the parabolic terminal/bounday value problem with continuous Dirichlet boundary condition). In addition to the hypotheses of Theorem 1.16, let $\mathscr{O} \subset \mathbb{H}$ be a domain such that the boundary $\Gamma_{1}$ obeys an exterior sphere condition, and $f \in C_{\mathrm{loc}}^{\alpha}(\bar{Q})$.

(1) If $\beta \geq 1$ and $g \in C_{\mathrm{loc}}\left(\overline{\overline{\mathrm{\partial}}^{1} Q}\right)$, then $u^{*}$ in (1.30) is a solution to problem (1.7) with boundary condition (1.9). In particular, $u^{*} \in C_{\mathrm{loc}}\left(Q \cup ð^{1} Q\right) \cap C^{2+\alpha}(Q)$ and obeys the growth assumption (1.29).

(2) If $0<\beta<1$ and $g \in C_{\mathrm{loc}}(\overline{\widetilde{Q} Q})$, then $u^{*}$ in (1.30) is a solution to problem (1.7) with boundary condition (1.12). In particular, $u^{*} \in C_{\mathrm{loc}}(Q \cup \partial Q) \cap$ $C^{2+\alpha}(Q)$ and satisfies the growth assumption (1.29).

For $T \varsubsetneqq \partial Q$ a relatively open subset, we let $C_{\text {loc }}^{2+\alpha}(Q \cup T)$ denote the subspace of $C^{2+\alpha}(Q)$ such that, for any precompact open set $U \Subset Q \cup T$, we have $u \in C^{2+\alpha}(\bar{U})$.

Theorem 1.23 (Existence of solutions to the parabolic terminal/boundary value problem (5.2) with $C^{2+\alpha}$ Dirichlet boundary condition). In addition to the hypotheses of Theorem 1.16, let $\mathscr{O} \subset \mathbb{H}$ be a domain such that the boundary portion $\Gamma_{1}$ is of class $C^{2+\alpha}$.

(1) If $\beta \geq 1$, the boundary portion $\Gamma_{1}$ is of class $C^{2+\alpha}$ and $g \in C_{\mathrm{loc}}^{2+\alpha}\left(Q \cup \varpi^{1} Q\right)$ obeys

$$
-g_{t}+A g=f \text { on }\{T\} \times \Gamma_{1} .
$$

Then $u^{*}$ in (1.30) is a solution to problem (5.2). In particular,

$$
u^{*} \in C_{\mathrm{loc}}^{2+\alpha}\left(Q \cup \mathrm{\delta}^{1} Q\right)
$$

and obeys the growth estimate (1.29).

(2) If $0<\beta<1$, the boundary portion $\Gamma_{1}$ is of class $C^{2+\alpha}$ and $g \in$ $C_{\text {loc }}^{2+\alpha}\left(Q \cup \partial^{1} Q\right) \cap C_{\text {loc }}(\bar{Q})$ obeys

$$
-g_{t}+A g=f \text { on }\{T\} \times \partial \mathscr{O} .
$$

Then $u^{*}$ in (1.30) is a solution to problem (5.2). In particular,

$$
u^{*} \in C_{\mathrm{loc}}^{2+\alpha}\left(Q \cup \mathrm{\partial}^{1} Q\right) \cap C_{\mathrm{loc}}(\bar{Q}),
$$

and obeys the growth estimate (1.29).

Remark 1.24 (Zero and first-order compatibility conditions for parabolic equations). The conditions (1.34) and (1.35) are the analogues of the first-order compatibility condition [34, Equation (10.4.3)]. The analogue of the zero-order compatibility condition in [34, Equation (10.4.2)] automatically holds at $\{T\} \times \Gamma_{1}$ or $\{T\} \times \partial \mathscr{O}$, since we always choose $h=g(T, \cdot)$ on $\Gamma_{1}$ or $\partial \mathscr{O}$, respectively, in this article.

Remark 1.25 (Existence of solutions with Daskalopoulos-Hamilton-Koch Hölder regularity). As in the elliptic case, the solutions to the parabolic terminal/boundary value problem (1.7), (1.9) should lie in $C_{s}^{2+\alpha}(\underline{Q}) \cap C_{\text {loc }}(\bar{Q})$ for all $\beta>0$, where $C_{s}^{2+\alpha}(Q)$ is the parabolic Daskalopoulos-Hamilton-Koch Hölder space described in [11, 33]. A function $u \in C_{s}^{2+\alpha}(\underline{Q})$ has the property that $u, D u, y D^{2} u$ are $C_{s}^{\alpha}$ 
continuous up to $\Gamma_{0}$ and $y D^{2} u=0$ on $(0, T) \times \Gamma_{0}$, where $C_{s}^{\alpha}(\underline{Q})$ is defined by analogy with the traditional definition [34] of $C^{\alpha}(Q)$, except that Euclidean distance between points in $Q$ is replaced by the cycloidal distance function. When $Q=$ $\mathbb{H} \times(0, T)$, we establish this existence result in [18, Theorem 1.1].

Remark 1.26 (Existence and uniqueness of solutions to parabolic terminal/boundary value problems). Existence and uniqueness of solutions to the parabolic terminal/boundary value problem (1.7) and (1.9), again provided $\Gamma_{1}=\partial \mathscr{O}$, follow from Schauder methods when the coefficient matrix, $\left(a^{i j}\right)$, of $A$ is strictly elliptic on $\overline{\mathscr{O}}$. For example, see [35, Theorems $5.9 \& 5.10]$ for the case where $f$ and the coefficients of $A$ are bounded and in $C^{\alpha}(Q)$, giving a unique solution $u \in C^{2+\alpha}(Q) \cap C(\bar{Q})$.

1.2.4. Uniqueness of solutions to parabolic obstacle problems. For $\theta_{1}, \theta_{2} \in \mathscr{T}_{t, T}$, $0 \leq t \leq T$, we set

$$
\begin{aligned}
J_{p}^{\theta_{1}, \theta_{2}}(t, z):= & \mathbb{E}_{\mathbb{Q}}^{t, z}\left[\int_{t}^{\theta_{1} \wedge \theta_{2}} e^{-r(s-t)} f(s, Z(s)) d s\right] \\
& +\mathbb{E}_{\mathbb{Q}}^{t, z}\left[e^{-r\left(\theta_{2}-t\right)} \psi\left(\theta_{2}, Z\left(\theta_{2}\right)\right) \mathbf{1}_{\left\{\theta_{2}<\theta_{1}\right\}}\right] \\
& +\mathbb{E}_{\mathbb{Q}}^{t, z}\left[e^{-r\left(\theta_{1}-t\right)} g\left(\theta_{1}, Z\left(\theta_{1}\right)\right) \mathbf{1}_{\left\{\theta_{1} \leq \theta_{2}\right\}}\right] .
\end{aligned}
$$

We have the following uniqueness result of solutions to the parabolic obstacle problem with different possible boundary conditions, depending on the value of the parameter $\beta>0$.

Theorem 1.27 (Uniqueness of solutions to the parabolic obstacle problem). Let $f$ be as in Theorem 1.16, and $\psi$ be a Borel measurable function satisfying (1.29).

(1) If $\beta \geq 1$, assume $\psi \in C_{\mathrm{loc}}\left(Q \cup \mathrm{\partial}^{1} Q\right)$ and $g \in C_{\mathrm{loc}}\left(\mathrm{\partial}^{1} Q\right)$ obey (1.29) on $\mathrm{\partial}^{1} Q$ and (1.11). Let

$$
u \in C_{\mathrm{loc}}\left(Q \cup \jmath^{1} Q\right) \cap C^{1,1}(Q)
$$

be a solution to the parabolic obstacle problem (1.8), (1.9) such that $u$ and $-u_{t}+$ Au obey (1.29) on $Q$. Then, $u=u^{*}$ on $Q \cup \partial^{1} Q$, where $u^{*}$ is given by

$$
u^{*}(t, z):=\sup _{\theta \in \mathscr{T}_{t, T}} J_{p}^{\tau_{\mathcal{O}} \wedge T, \theta}(t, z),
$$

and $\tau_{\mathscr{O}}$ is defined by (1.21), for all $(t, z) \in Q \cup \mathrm{J}^{1} Q$.

(2) If $0<\beta<1$, assume $\psi \in C_{\mathrm{loc}}(\bar{Q})$ and $g \in C_{\mathrm{loc}}(ð Q)$ obey (1.29) on ðQ and (1.14). Let

$$
u \in C_{\mathrm{loc}}(Q \cup \partial Q) \cap C^{1,1}(Q)
$$

be a solution to the parabolic obstacle problem (1.8), (1.12) such that $u$ and $-u_{t}+A u$ obey (1.29) on $Q$. Then, $u=u^{*}$ on $Q \cup \partial Q$, where $u^{*}$ is given by (1.37).

Theorem 1.28 (Uniqueness of solutions to the parabolic obstacle problem (1.8), (1.9), when $0<\beta<1)$. Let $0<\beta<1$ and $f$ be as in Theorem 1.18. Assume $\psi \in C_{\mathrm{loc}}(\bar{Q})$, and let $g \in C_{\mathrm{loc}}\left(\check{\partial}^{1} Q\right)$ obey (1.29) on $\check{\mathrm{d}}^{1} Q$ and (1.11). Let

$$
u \in C_{\mathrm{loc}}(\bar{Q}) \cap C_{s, \mathrm{loc}}^{1,1}\left(Q \cup(0, T) \times\left(\mathscr{O} \cup \Gamma_{0}\right)\right)
$$


be a solution to the parabolic obstacle problem (1.8), (1.9) such that $u$ and $-u_{t}+A u$ obey (1.29). Then, $u=u^{*}$ on $Q \cup \partial^{1} Q$, where $u^{*}$ is given by

$$
u^{*}(t, z):=\sup _{\theta \in \mathscr{T}_{t, T}} J_{p}^{\nu_{\mathscr{O}} \wedge T, \theta}(t, z),
$$

and $\nu_{\mathscr{O}}$ is defined by (1.22), for all $(t, z) \in Q \cup \check{\partial}^{1} Q$.

Remark 1.29 (Existence and uniqueness of strong solutions in weighted Sobolev spaces to the parabolic obstacle problem). Existence and uniqueness of strong solutions in weighted Sobolev spaces to problem (1.8) with Dirichlet boundary condition (1.9) along $\check{\partial}^{1} Q$, for all $\beta>0$, is proved in [10].

\subsection{Survey of previous results on stochastic representations of solutions} to boundary value or obstacle problems. Stochastic representations of solutions to elliptic and parabolic boundary value and obstacle problems discussed by Bensoussan and Lions [5] and Friedman [24] are established under the hypotheses that the matrix of coefficients, $\left(a^{i j}\right)$, of the second-order spatial derivatives in an elliptic linear, second-order differential operator, $A$, is strictly elliptic and that all coefficients of $A$ are bounded. Relaxations of these hypotheses, as in [24, Chapter 13 $\& 15]$, and more recently [47, fail to include the Heston generator mainly because the matrix $\left(a^{i j}\right)$ does not satisfy

Hypothesis 1.30 (Extension property for positive definite, $C^{2}$ matrix-valued functions). Given a subdomain $V \varsubsetneqq(0, \infty) \times \mathbb{R}^{d}$, for $d \geq 1$, we say that a matrix-valued function,

$$
a: V \rightarrow \mathbb{R}^{d \times d},
$$

which is $C^{2}$ on $V$ and $a(t, z)$ is positive definite for each $(t, z) \in V$ has the extension property if there is a matrix-valued function,

$$
\tilde{a}:[0, \infty) \times \mathbb{R}^{d} \rightarrow \mathbb{R}^{d \times d},
$$

which coincides with a on $V$ but is $C^{2}$ on $[0, \infty) \times \mathbb{R}^{d}$ and $\tilde{a}(t, z)$ is positive definite for each $(t, z) \in[0, \infty) \times \mathbb{R}^{d}$.

Naturally, Hypothesis 1.30 is also applicable when the matrix $a$ is constant with respect to time, that is, in elliptic problems. Note that in the case of the Heston process, $d=2, V=(0, \infty) \times \mathbb{H}$, and

$$
a(t, x, y):=\left(\begin{array}{ll}
y & \sigma \rho y \\
\sigma \rho y & \sigma^{2} y
\end{array}\right), \quad \forall(x, y) \in \mathbb{H}
$$

and so the matrix $a$ does not satisfy Hypothesis 1.30. We now give more detailed comparisons for each of the four main problems which we consider in this article.

1.3.1. Elliptic boundary value problems. Uniqueness of stochastic representations of solutions to non-degenerate elliptic partial differential equations is established in [24, Theorem 6.5.1], 30, Proposition 5.7.2], 38, Theorem 9.1.1 \& Corollary 9.1.2, Theorem 9.3.2], [5, Theorem 2.7.1 \& Remarks 2.7.1, 2.7.2] (for a bounded domain $\mathscr{O}$ ), and [5, Theorem 2.7.2\& Remarks 2.7.3-5] (when the domain is the whole space, $\left.\mathscr{O}=\mathbb{R}^{n}\right)$.

Existence (and uniqueness) of stochastic representations of solutions to nondegenerate elliptic partial differential equations is established in [24, Theorem 6.5.1], [38, Theorem 9.2.14], and [38, Theorem 9.3.3 \& Remark, p. 196]. 
Existence and uniqueness of solutions to a certain class of degenerate elliptic partial differential equations are described by Friedman in [24, Theorems 13.1.1 \& 13.3.1], but those results do not apply to the Heston operator because a square root, $\left(\sigma^{i j}\right)$, of the matrix $\left(a^{i j}\right)$ cannot be extended as a uniformly Lipschitz continuous function on $\mathbb{R}^{2}$, that is, 24, Condition (A), p. 308] is not satisfied. Stroock and Varadhan [43, §5-8] also discuss existence and uniqueness of solutions to degenerate elliptic partial differential equations, but their assumption that the matrix $\left(a^{i j}\right)$ satisfies Hypothesis 1.30 does not hold for the Heston operator (see 43, Theorem 2.1]).

More recently, Zhou [47] employs the method of quasiderivatives to establish the stochastic representation of solutions to a certain class of degenerate elliptic partial differential equations and obtains estimates for the derivatives of their solutions. However, his results do not apply to the Heston operator because [47, Assumptions 3.1 \& Condition (3.2)] are not satisfied in this case. Moreover, the Dirichlet condition is imposed on the whole boundary of the domain (see [47, Equation (1.1)]), while we take into consideration the portion of the boundary, $\Gamma_{0}$, where the differential operator $A$ becomes degenerate.

1.3.2. Elliptic obstacle problems. We may compare Theorems 1.12 and 1.13 with the uniqueness assertions (in increasing degrees of generality) for non-degenerate elliptic operators in 5. Theorems 3.3.1, 3.3.2, 3.3.4, 3.3.5, 3.3.8, 3.3.19, 3.3.20, \& 3.3.23]. See also [38, Theorem 10.4.1] and [24, Theorems 16.4.1, 16.4.2, 16.7.1, \& 16.8.1] for uniqueness assertions for non-degenerate elliptic operators, though with more limited applicability.

1.3.3. Parabolic boundary value problems. The uniqueness of solutions to nondegenerate parabolic partial differential equations and their stochastic representations are described in [24, Theorems 6.5.2, 6.5.3], [30, Theorem 5.7.6] and [5, Theorems $2.7 .3 \& 2.7 .4]$.

Friedman obtains fundamental solutions and stochastic representations of solutions to certain degenerate parabolic partial differential equations in [23], while he obtains uniqueness and stochastic representations of solutions to the Cauchy problem in 22]; those results are summarized in [24, Chapter 15]. Nevertheless, the results in [24, Chapter 15] and 23 do not apply to the Heston operator because Hypothesis 1.30 does not hold, that is, 24, Condition (A), p. 389] is not satisfied. Therefore, the method of construction in [23. Theorem 1.2] of a candidate for a fundamental solution does not apply to the Heston operator. A stochastic representation for a solution to the Cauchy problem for a degenerate operator is obtained in [24, §15.10], but the hypotheses of [24, Theorem 15.10.1] are again too restrictive and exclude the Heston operator.

Ekström and Tysk [14] consider the problem of pricing European-style options on an underlying process which is the solution to a degenerate, one-dimensional stochastic differential equation which satisfies [14, Hypothesis 2.1], and so includes the Feller square root (or Cox-Ingersoll-Ross) process, (2.1). The option price is the classical solution in the sense of [14, Definition 2.2] to the corresponding parabolic partial differential equation [14, Theorem 2.3]. Under their assumption that the payoff function $g(T, \cdot)$ is in $C^{1}([0, \infty))$, they show that their classical solution has the regularity property,

$$
u \in C([0, T] \times[0, \infty)) \cap C^{1}([0, T) \times[0, \infty)) \cap C^{2}([0, T) \times(0, \infty)),
$$


and obeys the second-order boundary condition,

$$
\lim _{(t, y) \rightarrow\left(0, t_{0}\right)} y u_{y y}(t, y)=0, \quad \forall t_{0} \in(0, T) \quad \text { (by [14, Proposition 4.1]). }
$$

As a consequence, in the framework of our article, their solution obeys

$$
u \in C_{s, \text { loc }}^{1,1}\left(\left(0, t_{0}\right) \times[0, \infty)\right), \quad \forall t_{0} \in(0, T),
$$

where the vector space of functions $C_{s, \text { loc }}^{1,1}\left(\left(0, t_{0}\right) \times[0, \infty)\right)$ is defined by analogy with (1.31).

In [13, Ekström and Tysk extend their results in [14] to the case of twodimensional stochastic volatility models for option prices, where the variance process satisfies the assumptions of [14, Hypothesis 2.1].

Bayraktar, Kardaras, and Xing [4] address the problem of uniqueness of classical solutions, in the sense of [4, Definitions $2.4 \& 2.5$ ], to a class of two-dimensional, degenerate parabolic partial differential equations. Their differential operator has a degeneracy which is similar to that of the Heston generator, $-A$, and to the differential operator considered in [14], but the matrix of coefficients, $\left(a^{i j}\right)$, of their operator may have more than quadratic growth with respect to the spatial variables (see 4, Standing Assumption 2.1]). Therefore, weak maximum principles for parabolic partial differential operators on unbounded domains such as [34, Exercise 8.1.22] do not guarantee uniqueness of solutions in such situations. The main result of their article - 4, Theorem 2.9] - establishes by probabilistic methods that uniqueness of classical solutions, obeying a natural growth condition, holds if and only if the asset price process is a martingale.

In our article, we consider the two-dimensional Heston stochastic process, (1.17), where the component $Y$ of the process satisfies [14, Hypothesis 2.1] and [4, Standing Assumption 2.1]. We only require the payoff function, $g(T, \cdot)$, to be continuous with respect to the spatial variables and have exponential growth, as in (1.29). Notice that the conditions on the payoff function are more restrictive in [14, Hypothesis 2.1] and 4, Standing Assumption 2.3] than in our article. We consider the parabolic equation associated to the Heston generator, $-A$, on bounded or unbounded subdomains, $\mathscr{O}$, of the upper half plane, $\mathbb{H}$, with Dirichlet boundary condition along the portion, $\Gamma_{1}$, of the boundary $\partial \mathscr{O}$ contained in $\mathbb{H}$. Along the portion, $\Gamma_{0}$, of the boundary contained in $\partial \mathbb{H}$, we impose a suitable Dirichlet boundary condition, depending on the value of the parameter $\beta$ in (1.18), which governs the behavior of the Feller square-root process when it approaches the boundary point $y=0$. In each case, we establish uniqueness of solutions by proving that suitably regular solutions must have the stochastic representations in Theorems 1.16 and 1.18, and we prove existence and regularity of solutions, in a special case, in Theorems 1.22 and 1.23, complementing the results of 14]. In addition, we consider the parabolic obstacle problem and establish uniqueness and the stochastic representations of suitably regular solutions in Theorems 1.27 and 1.28 .

1.3.4. Parabolic obstacle problems. We may compare Theorems 1.27 and 1.28 with the uniqueness assertions and stochastic representations of solutions (in increasing degrees of generality) for non-degenerate operators in [5, Theorems 3.4.1, 3.4.2, $3.4 .3,3.4 .5,3.4 .6,3.4 .7,3.4 .8]$.

1.4. Further work. The authors are developing an extension of the main results of this article to a broader class of degenerate Markov processes in higher dimensions 
and more general boundary conditions (including Neumann and oblique boundary conditions).

1.5. Outline of the article. For the convenience of the reader, we provide a brief outline of the article. We begin in 92 by reviewing or proving some of the key properties of the Feller square root and Heston processes which we shall need in this article. In 93 we prove existence and uniqueness (in various settings) of solutions to the elliptic boundary value problem for the Heston operator, while in $\$ 4$ we prove uniqueness (again in various settings) of solutions to the corresponding obstacle problem. We proceed in $\$ 5$ to prove existence and uniqueness of solutions to the parabolic terminal/boundary value problem for the Heston operator, and in $\$ 6$ we prove uniqueness of solutions to the corresponding parabolic obstacle problem. Appendices $\mathrm{A}$ and $\mathrm{B}$ contain additional technical results which we shall need throughout our article.

1.6. Notation and conventions. When we label a condition an Assumption, then it is considered to be universal and in effect throughout this article and so is not referenced explicitly in theorems and similar statements. When we label a condition a Hypothesis, then it is only considered to be in effect when explicitly referenced. We let $\mathbb{N}:=\{1,2,3, \ldots\}$ denote the set of positive integers. For $x, y \in \mathbb{R}$, we denote $x \wedge y:=\min \{x, y\}, x \vee y:=\max \{x, y\}$ and $x^{+}:=x \vee 0$.

\section{Properties of the Heston stochastic volatility process}

In this section, we review or develop some important properties of the Feller square root process and the Heston stochastic volatility process.

By [16. Theorem 1.11], it follows that for any initial point $(t, y) \in[0, \infty) \times[0, \infty)$, the Feller stochastic differential equation,

$$
\begin{aligned}
d Y(s) & =\kappa(\vartheta-Y(s)) d s+\sigma \sqrt{|Y(s)|} d W(s), \quad s>t, \\
Y(t) & =y,
\end{aligned}
$$

admits a unique weak solution $\left(Y^{t, y}(s), W(s)\right)_{s \geq t}$, called the Feller square root process, where $(W(s))_{s \geq t}$ is a one-dimensional Brownian motion on a filtered probability space $\left(\Omega, \mathscr{F}, \mathbb{P}^{t, y}, \mathbb{F}\right)$ such that the filtration $\mathbb{F}=\{\mathscr{F}(s)\}_{s \geq 0}$ satisfies the usual conditions [30, Definition 1.2.25]. Theorem 1.11 in 16 also implies that the Heston stochastic differential equation (1.17) admits a unique weak solution, $\left(Z^{t, z}(s), W(s)\right)_{s>t}$, for any initial point $(t, z) \in[0, \infty) \times \overline{\mathbb{H}}$, where $(W(s))_{s \geq t}$ is now an $\mathbb{R}^{2}$-valued Brownian motion on a filtered probability space $\left(\Omega, \mathscr{F}, \mathbb{Q}^{t, z}, \mathbb{F}\right)$ such that the filtration $\mathbb{F}=\{\mathscr{F}(s)\}_{s \geq 0}$ satisfies the usual conditions. When the initial condition $(t, y)$ or $(t, z)$ is clear from the context, we omit the superscripts in the definition of the probability measures $\mathbb{P}^{t, y}$ and $\mathbb{Q}^{t, z}$, respectively.

Moreover, the weak solutions to the Feller and Heston stochastic differential equations are strong. To prove this, we begin by reviewing a result of Yamada 46].

Definition 2.1 (Coefficients for a non-Lipschitz stochastic differential equation [46, p. 115]). In this article we shall consider one-dimensional stochastic differential equations whose diffusion and drift coefficients, $\alpha, b$, obey the following properties:

(1) The functions $\alpha, b:[0, \infty) \times \mathbb{R} \rightarrow \mathbb{R}$ are continuous. 
(2) (Yamada condition) There is an increasing function $\varrho:[0, \infty) \rightarrow[0, \infty)$ such that $\varrho(0)=0$, for some $\varepsilon>0$ one has $\int_{0}^{\varepsilon} \varrho^{-2}(y) d y=\infty$, and

$$
\left|\alpha\left(t, y_{1}\right)-\alpha\left(t, y_{2}\right)\right| \leq \varrho\left(\left|y_{1}-y_{2}\right|\right), \quad y_{1}, y_{2} \in \mathbb{R}, t \geq 0 .
$$

(3) There is a constant $C_{1}>0$ such that

$$
\left|b\left(t, y_{2}\right)-b\left(t, y_{1}\right)\right| \leq C_{1}\left|y_{2}-y_{1}\right|, \quad y_{1}, y_{2} \in \mathbb{R}, t \geq 0 .
$$

(4) There is a constant $C_{2}>0$ such that

$$
|\alpha(t, y)|+|b(t, y)| \leq C_{2}(1+|y|), \quad t \geq 0, y \in \mathbb{R} .
$$

Clearly, the coefficients of the Feller stochastic differential equation obey the hypotheses in Definition 2.1, where $\alpha(t, y)=\sigma \sqrt{y}$ and $b(t, y)=\kappa(\theta-y)$. Indeed, one can choose $C_{1}=\kappa, C_{2}=\max \{\kappa, \kappa \theta, \sigma\}$, and $\varrho(y)=\sigma \sqrt{y}$, as the mean value theorem yields

where

$$
\sqrt{y_{2}}-\sqrt{y_{1}}=c\left(y_{1}, y_{2}\right)\left(y_{2}-y_{1}\right)
$$

$$
c\left(y_{1}, y_{2}\right)=\frac{1}{2} \int_{0}^{1} \frac{1}{\sqrt{y_{1}+t\left(y_{2}-y_{1}\right)}} \leq \frac{1}{\sqrt{y_{2}-y_{1}}}
$$

for $0<y_{1}<y_{2}$. See [46, Remark 1] for other examples of suitable functions $\varrho$.

Remark 2.2. When $\varrho(u)=u^{\gamma}, \gamma \in[1 / 2,1]$ [46, Remark 1], then Definition 2.1 implies that $\alpha(t, \cdot)$ is Hölder continuous with exponent $\gamma$, uniformly with respect to $t \in[0, \infty)$.

Definition 2.3 (Solution to a non-Lipschitz stochastic differential equation [46, p. 115], [40, Definitions IX.1.2 \& IX.1.5]). Let $(\Omega, \mathscr{F}, \mathbb{P}, \mathbb{F})$ be a filtered probability space satisfying the usual conditions. We call a pair $(Y(s), W(s))_{s \geq 0}$ a weak solution to the non-Lipschitz one-dimensional stochastic differential equation,

$$
d Y(s)=b(s, Y(s)) d s+\alpha(s, Y(s)) d W(s), \quad s \geq 0, \quad Y(0)=y,
$$

where $y \in \mathbb{R}$, if the following hold:

(1) The processes $Y(s)$ and $W(s)$ are defined on $(\Omega, \mathscr{F}, \mathbb{P}, \mathbb{F})$.

(2) The process $Y(s)$ is continuous with respect to $s \in[0, \infty)$ and is $\mathbb{F}$-adapted.

(3) The process $W(s)$ is a standard $\mathbb{F}$-Brownian motion.

We call $(Y(s), W(s))_{s>0}$ a strong solution to (2.5) if $Y$ is $\mathbb{F}^{W}$-adapted, where $\mathbb{F}^{W}$ is the $\mathbb{P}$-completion of the filtration of $\mathscr{F}$ generated by $(W(s))_{s \geq 0}$. (Compare [28, Definition IV.1.2], [30, Definition 5.2.1], and [38, §5.3].)

Theorem 2.4 ([46, p. 117]). There exists a weak solution $(Y, W)$ to (2.5).

Remark 2.5. Yamada's main theorem [46, p. 117] asserts considerably more than Theorem 2.4. In particular, his article shows that (2.5) may be solved using the method of finite differences. Simpler results may suffice to merely guarantee the existence of a weak solution, as we need here; see Skorokhod [42.

Proposition 2.6. There exists a unique strong solution to (2.5).

Proof. Theorem 2.4 ensures that (2.5) admits a weak solution. Conditions (2.2) and (2.3) ensure that pathwise uniqueness holds for (weak) solutions to (2.5) by Revuz and Yor [40, Theorem IX.3.5 (ii)], while Karatzas and Shreve [30, Corollary 5.3.23] imply that (2.5) admits a strong solution; see [30, p. 310]. Conditions (2.2) and (2.3) guarantee the uniqueness of strong solutions to (2.5) by Karatzas and 
Shreve [30, Proposition 5.2.13]; compare Yamada and Watanabe 44,45]. (Pathwise uniqueness is also asserted for (2.5) by [28, Theorem IV.3.2] when (2.5) is timehomogeneous, noting that the coefficients $\alpha, b$ are not required to be bounded by Ikeda and Watanabe [28, p. 168].) We conclude that a strong solution to (2.5) exists and is unique.

Corollary 2.7. Given any initial point $(t, y) \in[0, \infty) \times[0, \infty)$, there exists a unique strong solution, $\left(Y^{t, y}(s), W(s)\right)_{s \geq t}$, to the Feller stochastic differential equation.

Proof. Immediate from Proposition 2.6.

Corollary 2.8. Given $(t, z) \in[0, \infty) \times \overline{\mathbb{H}}$, there exists a unique strong solution, $\left(Z^{t, z}(s), W(s)\right)_{s \geq t}$, to the Heston stochastic differential equation, where $(W(s))_{s \geq 0}$ is a standard two-dimensional Brownian motion on $\left(\Omega, \mathscr{F}, \mathbb{Q}, \mathbb{F}^{W}\right)$.

Proof. By Proposition 2.6, the Cox-Ingersoll-Ross stochastic differential equation has a unique strong solution, $\left(Y^{t, y}(s), W_{2}(s)\right)_{s \geq t}$, where $\left(W_{2}(s)\right)_{s \geq t}$ is a standard one-dimensional Brownian motion on $\left(\Omega, \mathscr{F}, \mathbb{P}, \mathbb{F}^{W_{2}}\right)$ and $\left(Y^{t, y}(s)\right)_{s \geq t}$ is $\mathbb{F}^{W_{2}}$ adapted. But given $\left(Y^{t, y}(s)\right)_{s \geq t}$ and a standard two-dimensional Brownian motion, $(W(s))_{s \geq t}=\left(W_{1}(s), W_{2}(s)\right)_{s \geq t}$ on $\left(\Omega, \mathscr{F}, \mathbb{Q}, \mathbb{F}^{W}\right)$, the process $\left(X^{t, x, y}(s)\right)_{s \geq t}$, and thus $\left(Z^{t, z}(s)\right)_{s \geq t}=\left(X^{t, x, y}(s), Y^{t, y}(s)\right)_{s \geq t}$, is uniquely determined by

$$
\begin{aligned}
X^{t, x, y}(s)= & x+\int_{t}^{s}\left(r-q-\frac{1}{2} Y^{t, y}(u)\right) d u \\
& +\int_{t}^{s} \sqrt{Y^{t, y}(u)}\left(\sqrt{1-\rho^{2}} d W_{1}(u)+\rho d W_{2}(u)\right) .
\end{aligned}
$$

This completes the proof.

Lemma 2.9 (Properties of the Feller square-root process). The unique strong solution of the Feller stochastic differential equation started at any $(t, y) \in[0, \infty) \times$ $[0, \infty)$ satisfies

$$
Y(s) \geq 0 \quad \mathbb{P}^{t, y} \text {-a.s., } \quad \forall s \geq t,
$$

and also

$$
\begin{aligned}
\int_{t}^{s} \mathbf{1}_{\{Y(u)=0\}} d u & =0, \quad \forall s \geq t, \\
L(s, x) & =0, \quad \forall x \leq 0, \forall s \geq t,
\end{aligned}
$$

where $L(\cdot, \cdot)$ is the local time of the Feller square-root process.

Proof. Without loss of generality, we may assume that $t=0$. In [4, Lemma 2.4] it is proved that $L(s, 0)=0$, for all $s \geq 0$, but it is not clear to us why it also follows that

$$
L(s, 0-):=\lim _{x \uparrow 0} L(s, x)=0, \quad \forall s \geq 0,
$$

a property we shall need in our proof of (2.6). To complete the argument, we consider the stochastic differential equation

$$
\begin{aligned}
d \widetilde{Y}(s) & =b(\widetilde{Y}(s)) d s+\alpha(\tilde{Y}(s)) d W(s), \quad s>0, \\
\widetilde{Y}(0) & =y
\end{aligned}
$$

where we let

$$
b(y):=\kappa(\vartheta-y) \text { and } \alpha(y):=\mathbf{1}_{\{y \geq 0\}} \sigma \sqrt{y}, \quad \forall y \in \mathbb{R} .
$$


This stochastic differential equation admits a unique strong solution by Proposition 2.6. We will show that $\widetilde{Y}(s) \geq 0$ a.s., for all $s \geq 0$, so that uniqueness of solutions to the Feller stochastic differential equation (2.1) implies that $\widetilde{Y}=Y$ a.s. and $Y$ will satisfy the same properties as $\tilde{Y}$. Thus, it is enough to prove (2.7) and (2.8) for $\tilde{Y}$. Property (2.6) is a consequence of the preceding two properties of $\tilde{Y}$.

Let $\widetilde{L}$ be the local time process for the continuous semimartingale $\widetilde{Y}$ (see 30 , Theorem 3.7.1]). From [30, Theorem 3.7.1 (iii)], we know that, for any Borel measurable function $k: \mathbb{R} \rightarrow[0, \infty)$, we have

$$
\int_{0}^{s} k(\widetilde{Y}(u)) \sigma^{2} \widetilde{Y}^{+}(u) d u=2 \int_{\mathbb{R}} k(x) \widetilde{L}(s, x) d x, \quad \forall s \geq 0 .
$$

Assume, to obtain a contradiction, that $\widetilde{L}(s, 0)>0$. From the right-continuity in the spatial variable of $\widetilde{L}(s, \cdot)$ [30, Theorem 3.7.1 (iv)], there are positive constants $c$ and $x_{0}$ such that $\widetilde{L}(s, x) \geq c$, for all $x \in\left[0, x_{0}\right]$. For $\varepsilon>0$, we define $k(x)=x^{-1}$, for $x \in\left[\varepsilon, x_{0}\right]$, and 0 otherwise. With this choice of $k$, the left-hand side in identity (2.10) is bounded in absolute value by $\sigma^{2} s$, for any $\varepsilon>0$, while the right-hand side of (2.10) is greater or equal than $2 c \log \left(x_{0} / \varepsilon\right)$, which diverges as $\varepsilon$ tends to 0 . Therefore, our assumption that $\widetilde{L}(s, 0)>0$ is false, and so $\widetilde{L}(s, 0)=0$. Moreover, we notice that for any bounded, Borel-measurable function $k$ with support in $(-\infty, 0)$ the left-hand side in identity (2.10) is identically zero. Thus, we conclude that $\widetilde{L}(s, x)=0$, for all $x<0$, and also $\widetilde{L}(s, 0-)=0$.

We use this result to show that $\mathbb{P}(\widetilde{Y}(s) \leq 0, \forall s \geq 0)=0$. From 30, p. 223, third formula] and the fact that $\kappa, \vartheta>0$, we see that

$$
0=\widetilde{L}(s, 0)-\widetilde{L}(s, 0-)=\kappa \vartheta \int_{0}^{s} \mathbf{1}_{\{\widetilde{Y}(u)=0\}} d u,
$$

which implies that $\mathbb{P}(\tilde{Y}(s)=0, \forall s \geq 0)=0$. It remains to show that $\mathbb{P}(\tilde{Y}(s) \in$ $(-\infty, 0))=0$, for all $s \geq 0$, which is equivalent to proving that for any $\varepsilon>0$ and $s \geq 0$, we have $\mathbb{P}(\tilde{Y}(s) \in(-\infty,-\varepsilon))=0$. Let $\varphi: \mathbb{R} \rightarrow[0,1]$ be a smooth cut-off function such that $\left.\varphi\right|_{(-\infty,-\varepsilon)} \equiv 1$ and $\left.\varphi\right|_{(0, \infty)} \equiv 0$. We can choose $\varphi$ such that $\varphi^{\prime} \leq 0$. Then, it follows by Itô's formula that

$$
\begin{aligned}
\varphi(\tilde{Y}(s))= & \varphi(\tilde{Y}(0))+\int_{0}^{s}\left(\kappa(\vartheta-\tilde{Y}(u)) \varphi^{\prime}(\tilde{Y}(u))+\frac{1}{2} \alpha^{2}(Y(u)) \varphi^{\prime \prime}(Y(u))\right) d u \\
& +\int_{0}^{s} \alpha(\tilde{Y}(u)) \varphi^{\prime}(\tilde{Y}(u)) d W(u) \\
= & \varphi(\tilde{Y}(0))+\int_{0}^{s} \kappa(\vartheta-\tilde{Y}(u)) \varphi^{\prime}(\tilde{Y}(u)) d u \quad\left(\text { as } \alpha(y)=0 \text { when } \varphi^{\prime} \neq 0\right) .
\end{aligned}
$$

We notice that the left-hand side is non-negative, while the right-hand side is nonpositive, as $\varphi^{\prime} \leq 0$ on $\mathbb{R}$ and $\varphi^{\prime}=0$ on $(0, \infty)$. Therefore, we must have $\varphi(\tilde{Y}(s))=0$ a.s., which implies that $\mathbb{P}(\tilde{Y}(s) \in(-\infty,-\varepsilon))=0$. This concludes the proof of the lemma.

For $a, y, t \geq 0$, we let

$$
T_{a}^{t, y}:=\inf \left\{s \geq t: Y^{t, y}(s)=a\right\}
$$


denote the first time the process $Y$ started at $y$ at time $t$ hits $a$. When the initial condition, $(t, y)$, is clear from the context, we omit the superscripts in the preceding definition (2.11). Also, when $t=0$, we omit the superscript $t$.

Lemma 2.10 (Boundary classification at $y=0$ of the Feller square root process). Let $Y^{y}$ be the unique strong solution to the Feller stochastic differential equation (2.1) with initial condition $Y^{y}(0)=y$. Then

(1) For $\beta \geq 1, y=0$ is an entrance boundary point in the sense of [32, §15.6(c)].

(2) For $0<\beta<1, y=0$ is a regular, instantaneously reflecting boundary point in the sense of [32, $\$ 15.6(\mathrm{a})]$, and

$$
\lim _{y \downarrow 0} T_{0}^{y}=0 \quad \text { a.s., }
$$

where $T_{0}^{y}$ is given by (2.11).

Proof. A direct calculation gives us that the scale function, $\mathfrak{s}$, and the speed measure, $\mathfrak{m}$, of the Feller square root process are given by

$$
\mathfrak{s}(y)=y^{-\beta} e^{\mu y} \text { and } \mathfrak{m}(y)=\frac{2}{\sigma^{2}} y^{\beta-1} e^{-\mu y}, \quad \forall y>0
$$

where $\beta=2 \kappa \vartheta / \sigma^{2}$ and $\mu=2 \kappa / \sigma^{2}$. We consider the following quantities, for $0<a<b<\infty$ and $x>0$ :

$$
\begin{aligned}
S[a, b] & :=\int_{a}^{b} \mathfrak{s}(y) d y, \quad S(a, b]:=\lim _{c \downarrow a} S[c, b], \\
M[a, b] & :=\int_{a}^{b} \mathfrak{m}(y) d y, \quad M(a, b]:=\lim _{c \downarrow a} M[c, b], \\
N(0) & :=\int_{0}^{x} S[y, x] \mathfrak{m}(y) d y .
\end{aligned}
$$

Then, for $\beta \geq 1$, we have $S(0, x]=\infty$ and $N(0)<\infty$, which implies that $y=0$ is an entrance boundary point ([32, p. 235]), while for $0<\beta<1$, we have $S(0, x]<\infty$ and $M(0, x]<\infty$, and so $y=0$ is a regular boundary point ([32, p. 232]).

Next, we consider the case $0<\beta<1$. To establish (2.12), we consider the following quantities:

$$
\begin{aligned}
u_{a, b}(y):=\mathbb{P}^{y}\left(T_{b}<T_{a}\right)= & \frac{S[a, y]}{S[a, b]}, \\
v_{a, b}(y):=\mathbb{E}_{\mathbb{P}}^{y}\left[T_{a} \wedge T_{b}\right]= & 2 u_{a, b}(y) \int_{y}^{b} S[z, b] m(z) d z \\
& +2\left(1-u_{a, b}(y)\right) \int_{a}^{y} S(a, z] m(z) d z,
\end{aligned}
$$

as in [32, Equations (15.6.1) \& (15.6.5)] and [32, Equations (15.6.2) \& (15.6.6)], respectively. Notice that $T_{a}^{y} \rightarrow T_{0}^{y}$, when $a \downarrow 0$, by the continuity of the paths of $Y$. Then, for fixed $b>0$, we obtain

$$
\begin{aligned}
& \lim _{y \downarrow 0} \mathbb{P}^{y}\left(T_{b}<T_{0}\right)=\lim _{y \downarrow 0} \lim _{a \downarrow 0} \mathbb{P}^{y}\left(T_{b}<T_{a}\right)=0, \\
& \lim _{y \downarrow 0} \mathbb{E}_{\mathbb{P}}^{y}\left[T_{0} \wedge T_{b}\right]=\lim _{y \downarrow 0} \lim _{a \downarrow 0} \mathbb{E}_{\mathbb{P}}^{y}\left[T_{a} \wedge T_{b}\right]=0,
\end{aligned}
$$

from where (2.12) follows. 
Next, we have the following

Lemma 2.11 (Properties of the Heston process). Let $(Z(s))_{s>0}$ be the unique strong solution to the Heston stochastic differential equation (1.17).

(1) Assume $q \geq 0$ and $r \in \mathbb{R}$. Then, for any constant $c \in[0,1]$,

$$
\left(e^{-r c s} e^{c X(s)}\right)_{s \geq 0} \text { is a positive supermartingale. }
$$

(2) For any positive constant $c \leq \mu$,

$$
\left(e^{-c \kappa \vartheta s} e^{c Y(s)}\right)_{s \geq 0} \text { is a positive supermartingale. }
$$

Proof. To establish (2.13), we use Itô's formula to give

$$
\begin{aligned}
d\left(e^{-r c s} e^{c X(s)}\right)= & -e^{-r c s} e^{c X(s)}\left(c q+\frac{1}{2} c(1-c) Y(s)\right) d s \\
& +c e^{-r c s} e^{c X(s)} \sqrt{Y(s)} d W_{1}(s) .
\end{aligned}
$$

Notice that the drift coefficient is non-positive, since $Y(s) \geq 0$ a.s. for all $s \geq 0$ by Lemma 2.9, and $q \geq 0$, and $c \in[0,1]$.

Similarly, to establish (2.14) for the Feller square root process, we have

$$
\begin{aligned}
d\left(e^{-c \kappa \vartheta s}\right. & \left.e^{c Y(s)}\right) \\
= & e^{-c \kappa \vartheta s} e^{c Y(s)} c\left(c \sigma^{2} / 2-\kappa\right) Y(s) d s \\
& +c \sigma e^{-c \kappa \vartheta s} e^{c Y(s)} \sqrt{Y(s)}\left(\rho d W_{1}(s)+\sqrt{1-\rho^{2}} d W_{2}(s)\right) .
\end{aligned}
$$

When $c \leq \mu$, we see that the drift coefficient in the preceding stochastic differential equation is non-negative.

The supermartingale properties (2.13) and (2.14) follow if we show in addition that the processes are integrable random variables for each time $s \geq 0$. For simplicity, we let $Q(s)$ denote either one of the processes we consider, and we let $\theta_{n}$ be the first exit time of the Heston process $(X(s), Y(s))_{s>0}$ from the rectangle $(-n, n) \times(0, n)$, where $n \in \mathbb{N}$. We set $Q_{n}(s):=Q\left(s \wedge \theta_{n}\right)$, for all $s \geq 0$. We then have

$$
d Q_{n}(s)=\mathbf{1}_{\left\{s \leq \theta_{n}\right\}} d Q_{n}(s), \quad \forall s>0, \quad \forall n \in \mathbb{N} .
$$

Using equations (2.15) and (2.16), it is clear that $\left(Q_{n}(s)\right)_{s \geq 0}$ are supermartingales, because the coefficients of the stochastic differential equations are bounded and the drift terms are non-positive. Therefore, we know that

$$
\mathbb{E}_{\mathbb{Q}}^{x, y}\left[Q_{n}(t) \mid \mathscr{F}(s)\right] \leq Q_{n}(s), \quad \forall t \geq s, \quad \forall s \geq 0, \quad \forall n \in \mathbb{N} .
$$

Clearly, we also have $Q_{n}(t) \rightarrow Q(t)$ a.s., as $n \rightarrow \infty$, for all $t \geq s$ and $s \geq 0$. Taking the limit as $n \rightarrow \infty$ in (2.17) and using the positivity of the processes, Fatou's lemma yields

$$
\begin{aligned}
\mathbb{E}_{\mathbb{Q}}^{x, y}[Q(t) \mid \mathscr{F}(s)] & \leq \liminf _{n \rightarrow \infty} \mathbb{E}_{\mathbb{Q}}^{x, y}\left[Q_{n}(t) \mid \mathscr{F}(s)\right] \\
& \leq \liminf _{n \rightarrow \infty} Q_{n}(s) \quad(\text { by }(2.17)) \\
& =Q(s), \quad \forall t \geq s, \quad \forall s \geq 0,
\end{aligned}
$$

and so (2.13) and (2.14) follow. 
The next lemma is used to show that the functions $u^{*}$ given by (1.23) and (1.25) are well defined and satisfy the growth assumption (1.20).

Lemma 2.12. Suppose $r>0$, and $f, g, \psi$ are Borel measurable functions on $\mathscr{O}$ and satisfy assumption (1.20). Then there is a positive constant $\bar{C}$, depending on $r, \kappa, \vartheta, M_{1}, M_{2}$ and $C$ in (1.20), such that for any $\theta_{1}, \theta_{2} \in \mathscr{T}$, the function $J_{e}^{\theta_{1}, \theta_{2}}$ in (1.26) satisfies the growth assumption,

$$
\left|J_{e}^{\theta_{1}, \theta_{2}}(x, y)\right| \leq \bar{C}\left(1+e^{M_{1} y}+e^{M_{2} x}\right), \quad \forall(x, y) \in \mathscr{O},
$$

where $0<M_{1}<\min \{r /(\kappa \vartheta), \mu\}$ and $M_{2} \in[0,1)$ are as in (1.20).

Remark 2.13. The obstacle function $\psi$ in (1.26) is only relevant for solutions to problem (1.2).

Proof. The conclusion is a consequence of the properties of the Heston process given in Lemma 2.11. We first estimate the integral term in (1.26). For $z \in \mathscr{O}$, then

$$
\begin{aligned}
\mathbb{E}_{\mathbb{Q}}^{z} & {\left[\int_{0}^{\theta_{1} \wedge \theta_{2}} e^{-r s}|f(Z(s))| d s\right] } \\
\leq & C \mathbb{E}_{\mathbb{Q}}^{z}\left[\int_{0}^{\infty} e^{-r s}\left(1+e^{-r s} e^{M_{1} Y(s)}+e^{-r s} e^{M_{2} X(s)}\right) d s\right] \\
\leq & C\left(1+\int_{0}^{\infty} e^{-\left(r-M_{1} \kappa \vartheta\right) s} \mathbb{E}_{\mathbb{Q}}^{z}\left[e^{-M_{1} \kappa \vartheta s} e^{M_{1} Y(s)}\right] d s\right. \\
& \left.+\int_{0}^{\infty} e^{-\left(1-M_{2}\right) r s} \mathbb{E}_{\mathbb{Q}}^{z}\left[e^{-r M_{2} s} e^{M_{2} X(s)} d s\right] d s\right) .
\end{aligned}
$$

Using the condition $M_{1}<\min \{r /(\kappa \vartheta), \mu\}$ and (2.14), together with $M_{2}<1$ and (2.13), we see that

$$
\mathbb{E}_{\mathbb{Q}}^{z}\left[\int_{0}^{\theta_{1} \wedge \theta_{2}} e^{-r s}|f(Z(s))| d s\right] \leq \bar{C}\left(1+e^{M_{1} y}+e^{M_{2} x}\right),
$$

for a positive constant $\bar{C}$ depending on $r, M_{1} \kappa \vartheta, M_{2}$ and the constant $C$ in the growth assumption (1.20) on $f, g$ and $\psi$.

Next, we show that the first non-integral term in (1.26) can be written as

$$
\mathbb{E}_{\mathbb{Q}}^{z}\left[e^{-r \theta_{1}} g\left(Z\left(\theta_{1}\right)\right) \mathbf{1}_{\left\{\theta_{1} \leq \theta_{2}\right\}}\right]=\mathbb{E}_{\mathbb{Q}}^{z}\left[e^{-r \theta_{1}} g\left(Z\left(\theta_{1}\right)\right) \mathbf{1}_{\left\{\theta_{1} \leq \theta_{2}, \theta_{1}<\infty\right\}}\right],
$$

for any $\theta_{1} \in \mathscr{T}$ which is not necessarily finite. This is reasonable because by rewriting

$$
\begin{aligned}
\mathbb{E}_{\mathbb{Q}}^{z}\left[e^{-r \theta_{1}} g\left(Z\left(\theta_{1}\right)\right) \mathbf{1}_{\left\{\theta_{1} \leq \theta_{2}\right\}}\right]= & \mathbb{E}_{\mathbb{Q}}^{z}\left[e^{-r \theta_{1}} g\left(Z\left(\theta_{1}\right)\right) \mathbf{1}_{\left\{\theta_{1} \leq \theta_{2} \wedge T\right\}}\right] \\
& +\mathbb{E}_{\mathbb{Q}}^{z}\left[e^{-r \theta_{1}} g\left(Z\left(\theta_{1}\right)\right) \mathbf{1}_{\left\{T<\theta_{1} \leq \theta_{2}\right\}}\right],
\end{aligned}
$$

we shall see that the second term converges to zero, as $T \rightarrow \infty$. Using the growth assumption on $g$ in (1.20), we have

$$
\begin{aligned}
& \mathbb{E}_{\mathbb{Q}}^{z}\left[e^{-r \theta_{1}}\left|g\left(Z\left(\theta_{1}\right)\right)\right| \mathbf{1}_{\left\{T<\theta_{1} \leq \theta_{2}\right\}}\right] \\
& \quad \leq C \mathbb{E}_{\mathbb{Q}}^{z}\left[e^{-r \theta_{1}}\left(1+e^{M_{1} Y\left(\theta_{1}\right)}+e^{M_{2} X\left(\theta_{1}\right)}\right) \mathbf{1}_{\left\{T<\theta_{1}\right\}}\right],
\end{aligned}
$$


and so by Lemma 2.11, we obtain

$$
\begin{aligned}
& \mathbb{E}_{\mathbb{Q}}^{z}\left[e^{-r \theta_{1}} g\left(Z\left(\theta_{1}\right)\right) \mathbf{1}_{\left\{T<\theta_{1} \leq \theta_{2}\right\}}\right] \\
& \quad \leq C\left(e^{-r T}+e^{-\left(r-M_{1} \kappa \vartheta\right) T} e^{M_{1} y}+e^{-r\left(1-M_{2}\right) T} e^{M_{2} x}\right) .
\end{aligned}
$$

Since $M_{1}<r /(\kappa \vartheta)$ and $M_{2}<1$, we see that the right-hand side converges to 0 , as $T \rightarrow \infty$. This justifies the identity (2.19).

Now, we use Fatou's lemma to obtain the bound (1.20) on the first non-integral term in (1.26). For $z \in \mathscr{O}$,

$$
\begin{aligned}
& \mathbb{E}_{\mathbb{Q}}^{z} {\left[e^{-r \theta_{1}}\left|g\left(Z\left(\theta_{1}\right)\right)\right| \mathbf{1}_{\left\{\theta_{1} \leq \theta_{2}\right\}}\right] } \\
& \quad \leq \liminf _{n \rightarrow \infty} \mathbb{E}_{\mathbb{Q}}^{z}\left[e^{-r\left(\theta_{1} \wedge n\right)}\left|g\left(Z\left(\theta_{1} \wedge n\right)\right)\right|\right] \\
& \quad \leq \liminf _{n \rightarrow \infty} C\left(1+\mathbb{E}_{\mathbb{Q}}^{z}\left[e^{-r\left(\theta_{1} \wedge n\right)} e^{M_{1} Y\left(\theta_{1} \wedge n\right)}\right]+\mathbb{E}_{\mathbb{Q}}^{z}\left[e^{-r\left(\theta_{1} \wedge n\right)} e^{M_{2} X\left(\theta_{1} \wedge n\right)}\right]\right)
\end{aligned}
$$

(by $(\underline{1.20})$ ).

Because $M_{1}<\mu$, we may apply the supermartingale property (2.14) with $c:=M_{2}$. We also use that $M_{1}<r /(\kappa \vartheta)$ to obtain $M_{1} \kappa \vartheta<r$, and so it follows by the Optional Sampling Theorem [30, Theorem 1.3.22] that

$$
\begin{aligned}
\mathbb{E}_{\mathbb{Q}}^{z}\left[e^{-r\left(\theta_{1} \wedge n\right)} e^{M_{1} Y\left(\theta_{1} \wedge n\right)}\right] & \leq \mathbb{E}_{\mathbb{Q}}^{z}\left[e^{-M_{1} \kappa \vartheta\left(\theta_{1} \wedge n\right)} e^{M_{1} Y\left(\theta_{1} \wedge n\right)}\right] \\
& \leq e^{M_{1} y}, \quad \forall n \in \mathbb{N} .
\end{aligned}
$$

Using the fact that $M_{2}<1$, we see by the supermartingale property that (2.13) applies with $c:=M_{1}$. By the Optional Sampling Theorem [30, Theorem 1.3.22] we have

$$
\begin{aligned}
\mathbb{E}_{\mathbb{Q}}^{z}\left[e^{-r\left(\theta_{1} \wedge n\right)} e^{M_{2} X\left(\theta_{1} \wedge n\right)}\right] & \leq \mathbb{E}_{\mathbb{Q}}^{z}\left[e^{-r M_{2}\left(\theta_{1} \wedge n\right)} e^{M_{2} X\left(\theta_{1} \wedge n\right)}\right] \\
& \leq e^{M_{2} x}, \quad \forall n \in \mathbb{N}
\end{aligned}
$$

Therefore, we obtain

$$
\mathbb{E}_{\mathbb{Q}}^{z}\left[e^{-r \theta_{1}}\left|g\left(Z\left(\theta_{1}\right)\right)\right| \mathbf{1}_{\left\{\theta_{1} \leq \theta_{2}\right\}}\right] \leq C\left(1+e^{M_{1} y}+e^{M_{2} x}\right) .
$$

We obtain the same bound on the second non-integral term in (1.26) because the obstacle function $\psi$ satisfies the same growth condition (1.20) as the boundary data $g$.

To prove Theorems 1.16 and 1.18 we make use of the following auxiliary result

Lemma 2.14. Let $z \in \overline{\mathbb{H}}$ and $T \in\left(0, T_{0}\right]$, where $T_{0}$ is a positive constant. Let $\left(Z^{z}(s)\right)_{s \geq 0}$ be the unique strong solution to the Heston stochastic differential equation (1.17) with initial condition $Z^{z}(0)=z$. Then there is a positive constant $c$, depending on $y, \kappa, \vartheta, \sigma$ and $T_{0}$, such that for any constant $p$ satisfying

$$
0 \leq p<\frac{c}{2 \sigma T},
$$

we have

$$
\sup _{\theta \in \mathscr{T}_{0, T}} \mathbb{E}_{\mathbb{Q}}^{z}\left[e^{p X^{z}(\theta)}\right]<\infty
$$

where $\mathscr{T}_{0, T}$ denotes the set of $\left(\Omega, \mathscr{F}, \mathbb{Q}^{z}, \mathbb{F}\right)$-stopping times with values in $[0, T]$. 
Proof. We use the method of time-change. Denote

$$
M_{i}(t):=\int_{0}^{t} \sqrt{Y(s)} d W_{i}(s), \quad i=1,2
$$

and observe that there is a two-dimensional Brownian motion $\left(B_{1}, B_{2}\right)[30$, Theorem 3.4.13] such that

$$
M_{i}(t)=B_{i}\left(\int_{0}^{t} Y(s) d s\right), \quad i=1,2 .
$$

Thus, we may rewrite the solution of the Heston stochastic differential equation (1.17) in the form

$$
\begin{array}{r}
X(t)=x+(r-q) s-\frac{1}{2} \int_{0}^{t} Y(s) d s+B_{1}\left(\int_{0}^{t} Y(s) d s\right), \\
Y(t)=y+\kappa \vartheta s-\kappa \int_{0}^{t} Y(s) d s+\sigma B_{3}\left(\int_{0}^{t} Y(s) d s\right),
\end{array}
$$

where $B_{3}:=\rho B_{1}+\sqrt{1-\rho^{2}} B_{2}$ is a one-dimensional Brownian motion.

For any continuous stochastic process $(P(t))_{t \geq 0}$, we let

$$
M_{P}(t):=\max _{0 \leq s \leq t} P(s), \quad \forall t \geq 0 .
$$

We first prove the following estimate.

Claim 2.15. There are positive constants $n_{0}$ and $c$, depending on $y, \kappa, \vartheta, \sigma$ and $T_{0}$, such that

$$
\mathbb{Q}^{z}\left(n \leq M_{Y}(T) \leq n+1\right) \leq \frac{2}{\sqrt{\pi}} e^{-c n /\left(2 \sigma^{2} T\right)} \mathbf{1}_{\left\{n \geq n_{0}\right\}}+\mathbf{1}_{\left\{n<n_{0}\right\}}, \quad \forall n \in \mathbb{N} .
$$

Proof. Notice that if $M_{Y}(T) \leq n+1$, where $n \in \mathbb{N}$, then

$$
\int_{0}^{T} Y(s) d s \leq(n+1) T
$$

and so, for any positive constant $m$,

$$
\begin{gathered}
\left\{\max _{0 \leq t \leq T} B_{3}\left(\int_{0}^{t} Y(s) d s\right) \geq m, M_{Y}(T) \leq n+1\right\} \\
\subseteq\left\{M_{B_{3}}((n+1) T) \geq m\right\} .
\end{gathered}
$$

Using the inclusion

$$
\left.\left\{n \leq M_{Y}(T)\right\} \subseteq\left\{\max _{0 \leq t \leq T} B_{3}\left(\int_{0}^{t} Y(s) d s\right) \geq \frac{n-y-\kappa \vartheta T}{\sigma}\right\} \quad \text { (by (2.23) }\right),
$$

we obtain by (2.25),

$$
\mathbb{Q}^{z}\left(n \leq M_{Y}(T) \leq n+1\right) \leq \mathbb{Q}^{z}\left(M_{B_{3}}((n+1) T) \geq \frac{n-y-\kappa \vartheta T}{\sigma}\right) .
$$

The expression for the density of the running maximum of Brownian motion [30. Equation (2.8.4)] yields

$$
\mathbb{Q}^{z}\left(M_{B_{3}}((n+1) T) \geq \frac{n-y-\kappa \vartheta T}{\sigma}\right) \leq \int_{(n-y-\kappa \vartheta T) /(\sigma \sqrt{(n+1) T})}^{\infty} \frac{2}{\sqrt{2 \pi}} e^{-x^{2} / 2} d x .
$$


As in 1, §7.1.2], we let

$$
\operatorname{erfc}(a):=\frac{2}{\sqrt{\pi}} \int_{a}^{\infty} e^{-x^{2} / 2} d x, \quad \forall a \in \mathbb{R},
$$

and so

$$
\mathbb{Q}^{z}\left(n \leq M_{Y}(T) \leq n+1\right) \leq \frac{1}{\sqrt{2}} \operatorname{erfc}\left(\frac{n-y-\kappa \vartheta T}{\sigma \sqrt{(n+1) T}}\right) .
$$

Because for any $a \geq 1$,

$$
\begin{aligned}
\int_{a}^{\infty} e^{-x^{2} / 2} d x & \leq \int_{a}^{\infty} x e^{-x^{2} / 2} d x \\
& =e^{-a^{2} / 2}
\end{aligned}
$$

we see that

$$
\operatorname{erfc}(a) \leq \frac{2}{\sqrt{\pi}} e^{-a^{2} / 2}, \quad \forall a \geq 1
$$

By hypothesis, $T \in\left(0, T_{0}\right]$, which implies that

$$
\frac{n-y-\kappa \vartheta T}{\sigma \sqrt{(n+1) T}} \geq \frac{n-y-\kappa \vartheta T_{0}}{\sigma \sqrt{(n+1) T_{0}}}, \quad \forall n \in \mathbb{N} .
$$

Hence, provided we have

$$
\frac{n-y-\kappa \vartheta T_{0}}{\sigma \sqrt{(n+1) T_{0}}} \geq 1
$$

which is true for all $n \geq n_{0}\left(y, \kappa, \vartheta, \sigma, T_{0}\right)$, the smallest integer such that the preceding inequality holds, we see that

$$
\mathbb{Q}^{z}\left(n \leq M_{Y}(T) \leq n+1\right) \leq \frac{2}{\sqrt{\pi}} e^{-(n-y-\kappa \vartheta T)^{2} /\left(2 \sigma^{2}(n+1) T\right)}, \quad \forall n \geq n_{0} .
$$

Similarly, for a possibly larger $n_{0}\left(y, \kappa, \vartheta, \sigma, T_{0}\right)$, again using the fact that $T \in\left(0, T_{0}\right]$, we may choose a positive constant $c$, depending also on $y, \kappa, \vartheta, \sigma$ and $T_{0}$, such that for all $n \geq n_{0}$, we have

$$
\frac{(n-y-\kappa \vartheta T)^{2}}{2 \sigma^{2}(n+1) T} \geq c \frac{n}{2 \sigma^{2} T} .
$$

Then, using the preceding inequality, we obtain the estimate (2.24) from (2.26). This completes the proof of the claim.

Next, we employ (2.24) to obtain (2.21). For any stopping time $\theta \in \mathscr{T}_{0, T}$, we may write

$$
e^{p X(\theta)}=\sum_{n=0}^{\infty} e^{p X(\theta)} \mathbf{1}_{\left\{M_{Y}(T) \leq n+1\right\}} \mathbf{1}_{\left\{n \leq M_{Y}(T) \leq n+1\right\}},
$$

and, by Hölder's inequality, it follows that

$$
\mathbb{E}_{\mathbb{Q}}^{z}\left[e^{p X(\theta)}\right] \leq \sum_{n=0}^{\infty} \mathbb{E}_{\mathbb{Q}}^{z}\left[e^{2 p X(\theta)} \mathbf{1}_{\left\{M_{Y}(T) \leq n+1\right\}}\right]^{1 / 2} \mathbb{Q}^{z}\left(n \leq M_{Y}(T) \leq n+1\right)^{1 / 2}
$$


Using (2.22) and the condition $p \geq 0$ in (2.20), we have

$$
\begin{aligned}
& \mathbb{E}_{\mathbb{Q}}^{z} {\left[e^{2 p X(\theta)} \mathbf{1}_{\left\{M_{Y}(T) \leq n+1\right\}}\right] } \\
& \leq e^{2 p(x+|r-q| T)} \mathbb{E}_{\mathbb{Q}}^{z}\left[\exp \left(2 p B_{1}\left(\int_{0}^{\theta} Y(s) d s\right)\right) \mathbf{1}_{\left\{M_{Y}(T) \leq n+1\right\}}\right] \\
& \quad \leq e^{2 p(x+|r-q| T)} \mathbb{E}_{\mathbb{Q}}^{z}\left[\exp \left(2 p \max _{0 \leq t \leq T} B_{1}\left(\int_{0}^{t} Y(s) d s\right)\right) \mathbf{1}_{\left\{M_{Y}(T) \leq n+1\right\}}\right] \\
& \leq e^{2 p(x+|r-q| T)} \mathbb{E}_{\mathbb{Q}}^{z}\left[e^{2 p M_{B_{1}}((n+1) T)}\right], \quad \forall n \in \mathbb{N} \quad(\text { by }(\underline{2.25}) .
\end{aligned}
$$

We see from the expression for the density of the running maximum of Brownian motion [30, Exercise (2.8.4)] that

$$
\begin{aligned}
\mathbb{E}_{\mathbb{Q}}^{z}\left[e^{2 p M_{B_{1}}((n+1) T)}\right] & =\int_{0}^{\infty} e^{2 p x} \frac{2}{\sqrt{2 \pi(n+1) T}} e^{-x^{2} /(2(n+1) T)} d x \\
& \leq 2 e^{2 p^{2}(n+1) T}, \quad \forall n \in \mathbb{N} \quad \text { (by Mathematica) },
\end{aligned}
$$

and so

$$
\mathbb{E}_{\mathbb{Q}}^{z}\left[e^{2 p X(\theta)} \mathbf{1}_{\left\{M_{Y}(T) \leq n+1\right\}}\right] \leq 2 e^{2 p(x+|r-q| T)} e^{2 p^{2}(n+1) T}, \quad \forall n \in \mathbb{N} .
$$

Inequalities (2.24), (2.27) and (2.28) give us

$$
\begin{aligned}
\mathbb{E}_{\mathbb{Q}}^{z}\left[e^{p X(\theta)}\right] \leq & \sqrt{2} e^{p(x+|r-q| T)} \sum_{n=0}^{n_{0}-1} e^{p^{2}(n+1) T} \\
& +\frac{2}{\pi^{1 / 4}} e^{p(x+|r-q| T)} \sum_{n=n_{0}}^{\infty} e^{p^{2}(n+1) T} e^{-c n /\left(4 \sigma^{2} T\right)} \\
= & \sqrt{2} e^{p(x+|r-q| T)} \sum_{n=0}^{n_{0}-1} e^{p^{2}(n+1) T} \\
& +\frac{2}{\pi^{1 / 4}} e^{p(x+|r-q| T)+p^{2} T} \sum_{n=n_{0}}^{\infty} e^{\left(p^{2} T-c /\left(4 \sigma^{2} T\right)\right) n} .
\end{aligned}
$$

We choose $p$ such that

$$
0 \leq p<\frac{\sqrt{c}}{2 \sigma T}
$$

that is, condition $(2.20)$ is obeyed, and we obtain a bound on $\mathbb{E}_{\mathbb{Q}}^{z}\left[e^{p X(\theta)}\right]$ which is independent of the choice of $\theta \in \mathscr{T}_{0, T}$. Thus, (2.21) follows. (Note that (2.21) holds trivially when $p=0$.)

\section{Elliptic Boundary VAlue PROBlem}

In this section, we prove Theorem 1.3. In addition to the uniqueness result in Theorem 1.3 we establish the existence and uniqueness of solutions in Theorem 1.8.

The existence and uniqueness of solutions to problem (1.1) with boundary condition (1.3) along $\Gamma_{1}$, when $\beta \geq 1$, and with boundary condition (1.5) along $\partial \mathscr{O}$, when $0<\beta<1$, are similar in nature. Therefore, we define

$$
\partial_{\beta} \mathscr{O}:= \begin{cases}\Gamma_{1} & \text { if } \beta \geq 1, \\ \partial \mathscr{O} & \text { if } 0<\beta<1\end{cases}
$$


and treat the previously mentioned boundary value problems together as

$$
\begin{cases}A u=f & \text { on } \mathscr{O} \\ u=g & \text { on } \partial_{\beta} \mathscr{O} .\end{cases}
$$

Now, we can give the

Proof of Theorem 1.3. Our goal is to show that if $u \in C_{\text {loc }}\left(\mathscr{O} \cup \partial_{\beta} \mathscr{O}\right) \cap C^{1,1}(\mathscr{O})$ is a solution to problem (3.2), satisfying the pointwise growth condition (1.20), then it admits the stochastic representation (1.23).

We let $\left\{\mathscr{O}_{k}: k \in \mathbb{N}\right\}$ denote an increasing sequence of $C^{2+\alpha}$ subdomains of $\mathscr{O}$ (see [25, Definition §6.2]) such that each $\mathscr{O}_{k}$ has compact closure in $\mathscr{O}$, and

$$
\bigcup_{k \in \mathbb{N}} \mathscr{O}_{k}=\mathscr{O} \text {. }
$$

By applying Itô's lemma [5, Theorem 2.8.5] with $p=\infty$, we obtain for all $t>0$,

$$
\mathbb{E}_{\mathbb{Q}}^{z}\left[e^{-r\left(t \wedge \tau_{\mathscr{O}_{k}}\right)} u\left(Z\left(t \wedge \tau_{\mathscr{O}_{k}}\right)\right)\right]=u(z)-\mathbb{E}_{\mathbb{Q}}^{z}\left[\int_{0}^{t \wedge \tau_{\mathscr{C}_{k}}} e^{-r s} f(Z(s)) d s\right]
$$

We take the limit as $k$ tends to $\infty$ in the preceding identity. By the growth estimate (2.18), we may apply the Lebesgue Dominated Convergence Theorem to show that the integral term in (3.3) converges to

$$
\mathbb{E}_{\mathbb{Q}}^{z}\left[\int_{0}^{t \wedge \tau_{\mathscr{O}}} e^{-r s} f(Z(s)) d s\right]
$$

For the non-integral term on the left-hand side of (3.3), using the continuity of $u$ on $\mathscr{O} \cup \partial_{\beta} \mathscr{O}$ and of the sample paths of the Heston process, we see that

$$
e^{-r\left(t \wedge \tau_{\mathscr{O}_{k}}\right)} u\left(Z\left(t \wedge \tau_{\mathscr{O}_{k}}\right)\right) \rightarrow e^{-r\left(t \wedge \tau_{\mathscr{C}}\right)} u\left(Z\left(t \wedge \tau_{\mathscr{O}}\right)\right), \quad \text { a.s. as } k \rightarrow \infty .
$$

Using [6, Theorem 16.13], we prove that

$$
\mathbb{E}_{\mathbb{Q}}^{z}\left[e^{-r\left(t \wedge \tau_{\mathscr{O}_{k}}\right)} u\left(Z\left(t \wedge \tau_{\mathscr{O}_{k}}\right)\right)\right] \rightarrow \mathbb{E}_{\mathbb{Q}}^{z}\left[e^{-r\left(t \wedge \tau_{\mathscr{O}}\right)} u\left(Z\left(t \wedge \tau_{\mathscr{O}}\right)\right)\right], \quad \text { as } k \rightarrow \infty,
$$

by showing that

$$
\left\{e^{-r\left(t \wedge \tau_{\mathscr{O}_{k}}\right)} u\left(Z\left(t \wedge \tau_{\mathscr{O}_{k}}\right)\right): k \in \mathbb{N}\right\}
$$

is a collection of uniformly integrable random variables. By 6, Remark related to formula (16.23)], it suffices to show that their $p$-th order moment is uniformly bounded (independent of $k$ ), for some $p>1$. We choose $p>1$ such that $p M_{1}<\mu$ and $p M_{2}<1$. Notice that this is possible because we assumed the coefficients $M_{1}<\mu$ and $M_{2}<1$. Then, from the growth estimate (1.20), we have

$$
\left|e^{-r\left(t \wedge \tau_{\mathscr{O}_{k}}\right)} u(Z)\right|^{p} \leq C e^{-r p\left(t \wedge \tau_{\mathscr{O}_{k}}\right)}\left(1+e^{p M_{1} Y}+e^{p M_{2} X}\right), \quad \forall k \in \mathbb{N} .
$$

From property (2.14) applied with $c=p M_{1}<\mu$, and property (2.13) applied with $c=p M_{2} \in(0,1)$, we obtain using the fact that $M_{1}<r /(\kappa \vartheta)$,

$$
\mathbb{E}_{\mathbb{Q}}^{z}\left[\left|e^{-r\left(t \wedge \tau_{\mathscr{\sigma}_{k}}\right)} u\left(Z\left(t \wedge \tau_{\mathscr{O}_{k}}\right)\right)\right|^{p}\right] \leq C\left(1+e^{p M_{1} y}+e^{p M_{2} x}\right), \quad \forall k \in \mathbb{N} .
$$

Therefore, by taking the limit as $k$ tends to $\infty$ in (3.3) we obtain

$$
\mathbb{E}_{\mathbb{Q}}^{z}\left[e^{-r\left(t \wedge \tau_{\mathscr{O}}\right)} u\left(Z\left(t \wedge \tau_{\mathscr{O}}\right)\right)\right]=u(z)-\mathbb{E}_{\mathbb{Q}}^{z}\left[\int_{0}^{t \wedge \tau_{\mathscr{O}}} e^{-r s} f(Z(s)) d s\right]
$$


As we let $t$ tend to $\infty$, the integral term on the right-hand side in the preceding identity clearly converges to

$$
\mathbb{E}_{\mathbb{Q}}^{z}\left[\int_{0}^{\tau_{\mathscr{O}}} e^{-r s} f(Z(s)) d s\right]
$$

It remains to consider the left-hand side of (3.4). Keeping in mind that $u \in$ $C_{\text {loc }}\left(\mathscr{O} \cup \partial_{\beta} \mathscr{O}\right)$ solves (3.2), we rewrite this term as

$$
\begin{aligned}
\mathbb{E}_{\mathbb{Q}}^{z}\left[e^{-r\left(t \wedge \tau_{\mathscr{O}}\right)} u\left(Z\left(t \wedge \tau_{\mathscr{O}}\right)\right)\right]= & \mathbb{E}_{\mathscr{Q}}^{z}\left[e^{-r \tau_{\mathscr{O}}} g\left(Z\left(\tau_{\mathscr{O}}\right)\right) \mathbf{1}_{\left\{\tau_{\mathscr{O}} \leq t\right\}}\right] \\
& +\mathbb{E}_{\mathbb{Q}}^{z}\left[e^{-r t} u(Z(t)) \mathbf{1}_{\left\{\tau_{\mathscr{O}}>t\right\}}\right] .
\end{aligned}
$$

Using the growth assumption (1.20), we notice as above that both collections of random variables in the preceding identity,

$$
\left\{e^{-r \tau_{\mathscr{O}}} g\left(Z\left(\tau_{\mathscr{O}}\right)\right) \mathbf{1}_{\left\{\tau_{\mathscr{O}} \leq t\right\}}: t \geq 0\right\} \text { and }\left\{e^{-r t} u(Z(t)) \mathbf{1}_{\left\{\tau_{\mathscr{O}}>t\right\}}: t \geq 0\right\},
$$

are uniformly integrable, and they converge a.s. to $e^{-r \tau_{\mathscr{O}}} g\left(Z\left(\tau_{\mathscr{O}}\right)\right) \mathbf{1}_{\left\{\tau_{\mathscr{O}}<\infty\right\}}$ and zero, respectively. Therefore, by [6, Theorem 16.13], letting $t$ tend to $\infty$ in (3.4), we obtain

$$
\mathbb{E}_{\mathbb{Q}}^{z}\left[e^{-r \tau_{\mathscr{O}}} g\left(Z\left(\tau_{\mathscr{O}}\right)\right) \mathbf{1}_{\left\{\tau_{\mathscr{O}}<\infty\right\}}\right]=u(z)-\mathbb{E}_{\mathbb{Q}}^{z}\left[\int_{0}^{\tau_{\mathscr{O}}} e^{-r s} f(Z(s)) d s\right],
$$

which implies that $u=u^{*}$ on $\mathscr{O} \cup \partial_{\beta} \mathscr{O}$, where $u^{*}$ is defined by (1.23).

Proof of Theorem 1.5. Our goal is to show that if $0<\beta<1$ and $u \in C_{\text {loc }}\left(\mathscr{O} \cup \Gamma_{1}\right) \cap$ $C_{s, \text { loc }}^{1,1}\left(\mathscr{O} \cup \Gamma_{0}\right)$ is a solution to problem (1.1), satisfying the growth estimate (1.20), then it admits the stochastic representation (1.25).

We consider the following sequence of increasing subdomains of $\mathscr{O}$,

$$
\mathscr{U}_{k}:=\left\{z \in \mathscr{O}:|z|<k, \operatorname{dist}\left(z, \Gamma_{1}\right)>1 / k\right\}, \quad k \in \mathbb{N},
$$

with non-empty boundary portions $\bar{\Gamma}_{0} \cap \overline{\mathscr{U}}_{k}$. Let $\varepsilon>0$ and denote

$$
Y^{\varepsilon}:=Y+\varepsilon \text { and } Z^{\varepsilon}:=\left(X, Y^{\varepsilon}\right) \text {. }
$$

By applying Itô's lemma [5, Theorem 2.8.5] with $p=\infty$, we obtain

$$
\begin{aligned}
\mathbb{E}_{\mathbb{Q}}^{z}\left[e^{-r\left(t \wedge \nu \mathscr{\mathscr { U }}_{k}\right)} u\left(Z^{\varepsilon}\left(t \wedge \nu_{\mathscr{U}_{k}}\right)\right)\right] \\
\quad=u(z)-\mathbb{E}_{\mathbb{Q}}^{z}\left[\int_{0}^{t \wedge \nu \mathscr{U}_{k}} e^{-r s} A^{\varepsilon} u\left(Z^{\varepsilon}(s)\right) d s\right], \quad \forall t>0,
\end{aligned}
$$

where $\nu \mathscr{U}_{k}$ is given by (1.22), and $A^{\varepsilon}$ denotes the elliptic differential operator,

$$
-A^{\varepsilon} v:=-A v+\frac{\varepsilon}{2} v_{x}+\kappa \varepsilon v_{y}-\frac{\varepsilon}{2}\left(v_{x x}+2 \rho \sigma v_{x y}+\sigma^{2} v_{y y}\right), \quad \forall v \in C^{2}(\mathscr{O}) .
$$

To prove (3.7), we apply Itô's lemma to $u\left(Z^{\varepsilon}(t)\right)$ and we obtain

$$
\begin{aligned}
& d e^{-r\left(t \wedge \nu \mathscr{U}_{k}\right)} u\left(Z^{\varepsilon}\left(t \wedge \nu \mathscr{U}_{k}\right)\right) \\
&= e^{-r t} 1_{\left\{t \leq \nu \mathscr{U}_{k}\right\}}\left(u_{x}\left(Z^{\varepsilon}(t)\right) d X(t)+u_{y}\left(Z^{\varepsilon}(t)\right) d Y(t)\right. \\
&+\frac{1}{2} u_{x x}\left(Z^{\varepsilon}(t)\right) d[X, X](t)+u_{x y}\left(Z^{\varepsilon}(t)\right) d[X, Y](t) \\
&\left.+\frac{1}{2} u_{y y}\left(Z^{\varepsilon}(t)\right) d[Y, Y](t)\right) .
\end{aligned}
$$


Consequently,

$$
\begin{aligned}
& d e^{-r\left(t \wedge \nu \mathscr{U}_{k}\right)} u\left(Z^{\varepsilon}\left(t \wedge \nu \mathscr{U}_{k}\right)\right) \\
&= e^{-r t} 1_{\left\{t \leq \nu \mathscr{U}_{k}\right\}}\left(\left(r-q-\frac{1}{2}(Y(t)+\varepsilon)\right) u_{x}\left(Z^{\varepsilon}(t)\right)\right. \\
&+\kappa(\theta-(Y(t)+\varepsilon)) u_{y}\left(Z^{\varepsilon}(t)\right) \\
&+\frac{1}{2}(Y(t)+\varepsilon) u_{x x}\left(Z^{\varepsilon}(t)\right)+\sigma \rho(Y(t)+\varepsilon) u_{x y}\left(Z^{\varepsilon}(t)\right) \\
&+\frac{1}{2} \sigma^{2}(Y(t)+\varepsilon) u_{y y}\left(Z^{\varepsilon}(t)\right)+\frac{\varepsilon}{2} u_{x}\left(Z^{\varepsilon}(t)\right)+\varepsilon \kappa u_{y}\left(Z^{\varepsilon}(t)\right) \\
&\left.-\frac{\varepsilon}{2} u_{x x}\left(Z^{\varepsilon}(t)\right)-\varepsilon \sigma \rho u_{x y}\left(Z^{\varepsilon}(t)\right)-\frac{\varepsilon}{2} \sigma^{2} u_{y y}\left(Z^{\varepsilon}(t)\right)\right) d t \\
&+e^{-r t} 1_{\left\{t \leq \nu \mathscr{U}_{k}\right\}} \sqrt{Y(t)}\left[\left(u_{x}\left(Z^{\varepsilon}(t)\right)+\rho \sigma u_{y}\left(Z^{\varepsilon}(t)\right)\right) d W_{1}(t)\right. \\
&\left.+\sigma \sqrt{1-\rho^{2}} u_{y}\left(Z^{\varepsilon}(t)\right) d W_{2}(t)\right] .
\end{aligned}
$$

By (3.8) and (1.15), the preceding equality becomes

$$
\begin{aligned}
& d e^{-r\left(t \wedge \nu \mathscr{U}_{k}\right)} u\left(Z^{\varepsilon}\left(t \wedge \nu \mathscr{U}_{k}\right)\right) \\
& =e^{-r t} 1_{\left\{t \leq \nu \mathscr{U}_{k}\right\}}\left(-A^{\varepsilon}\right) u\left(Z^{\varepsilon}(t)\right) d t \\
& \quad+e^{-r t} 1_{\left\{t \leq \nu \mathscr{U}_{k}\right\}} \sqrt{Y(t)}\left[\left(u_{x}\left(Z^{\varepsilon}(t)\right)+\rho \sigma u_{y}\left(Z^{\varepsilon}(t)\right)\right) d W_{1}(t)\right. \\
& \left.\quad+\sigma \sqrt{1-\rho^{2}} u_{y}\left(Z^{\varepsilon}(t)\right) d W_{2}(t)\right] .
\end{aligned}
$$

Because $u \in C_{s, \text { loc }}^{1,1}\left(\mathscr{O} \cup \Gamma_{0}\right)$ and the fact that the stopped process $\left\{Z\left(t \wedge \nu \mathscr{U}_{k}\right)\right\}$ is constrained to stay in the set $\overline{\mathscr{U}}_{k}$, the $d W_{1}(t)$ and $d W_{2}(t)$ terms in the preceding expression are martingales.

Using (1.1), we can write (3.7) as

$$
\begin{aligned}
\mathbb{E}_{\mathbb{Q}}^{z}\left[e^{-r\left(t \wedge \nu \mathscr{U}_{k}\right)} u\left(Z^{\varepsilon}\left(t \wedge \nu \mathscr{U}_{k}\right)\right)\right]= & u(x, y+\varepsilon)-\mathbb{E}_{\mathbb{Q}}^{z}\left[\int_{0}^{t \wedge \nu \mathscr{U}_{k}} e^{-r s} f\left(Z^{\varepsilon}(s)\right) d s\right] \\
& -\mathbb{E}_{\mathbb{Q}}^{z}\left[\int_{0}^{t \wedge \nu \mathscr{U}_{k}} e^{-r s}\left(A^{\varepsilon}-A\right) u\left(Z^{\varepsilon}(s)\right) d s\right] .
\end{aligned}
$$

First, we take the limit as $\varepsilon$ tends to 0 in the preceding identity. Because $z \in \mathscr{O} \cup \Gamma_{1}$ and $u \in C\left(\mathscr{O} \cup \Gamma_{1}\right)$, we see that

$$
u(x, y+\varepsilon) \rightarrow u(x, y)=u(z), \quad \text { as } \varepsilon \downarrow 0 .
$$

We may assume without loss of generality that $\varepsilon<1 / k$, for any fixed $k \geq 1$. We evaluate the residual term $\left(A^{\varepsilon}-A\right) u$ with (3.8) to give

$$
\begin{aligned}
\left|\left(A^{\varepsilon}-A\right) u\left(Z^{\varepsilon}(s)\right)\right| \leq & C \varepsilon\|D u\|_{C\left(\overline{\mathscr{U}}_{2 k}\right)} \\
& +C\left(\mathbf{1}_{\left\{Y^{\varepsilon}(s) \leq \sqrt{\varepsilon}\right\}}+\sqrt{\varepsilon}\right)\left\|y D^{2} u\right\|_{C\left(\overline{\mathscr{U}}_{2 k}\right)},
\end{aligned}
$$

for all $0 \leq s \leq t \wedge \nu \mathscr{U}_{k}$, where $C$ is a positive constant depending only on the Heston constant coefficients. This follows from the fact that

$$
\varepsilon D^{2} u\left(Z^{\varepsilon}(s)\right)=\varepsilon D^{2} u\left(Z^{\varepsilon}(s)\right) \mathbf{1}_{\left\{Y^{\varepsilon}(s) \leq \sqrt{\varepsilon}\right\}}+\varepsilon D^{2} u\left(Z^{\varepsilon}(s)\right) \mathbf{1}_{\left\{Y^{\varepsilon}(s)>\sqrt{\varepsilon}\right\}}, \quad \forall s \geq 0
$$


and so

$$
\begin{aligned}
\varepsilon\left|D^{2} u\left(Z^{\varepsilon}(s)\right)\right| & \leq Y^{\varepsilon}(s)\left|D^{2} u\left(Z^{\varepsilon}(s)\right)\right| \mathbf{1}_{\left\{Y^{\varepsilon}(s) \leq \sqrt{\varepsilon}\right\}}+\varepsilon \frac{Y^{\varepsilon}(s)}{\sqrt{\varepsilon}}\left|D^{2} u\left(Z^{\varepsilon}(s)\right)\right| \mathbf{1}_{\left\{Y^{\varepsilon}(s)>\sqrt{\varepsilon}\right\}} \\
& \leq\left(\mathbf{1}_{\left\{Y^{\varepsilon}(s) \leq \sqrt{\varepsilon}\right\}}+\sqrt{\varepsilon}\right) Y^{\varepsilon}(s)\left|D^{2} u\left(Z^{\varepsilon}(s)\right)\right| .
\end{aligned}
$$

Combining the preceding inequality with the definition (3.8) of $A^{\varepsilon}$, we obtain (3.11). Since $u \in C_{s, l o c}^{1,1}\left(\mathscr{O} \cup \Gamma_{0}\right)$ and

$$
\mathbf{1}_{\left\{Y^{\varepsilon}(s) \leq \sqrt{\varepsilon}\right\}} \rightarrow 0, \quad \text { as } \varepsilon \downarrow 0,
$$

we see that (3.11) yields, for each $k \geq 1$,

$$
\mathbb{E}_{\mathbb{Q}}^{z}\left[\int_{0}^{t \wedge \nu_{\mathscr{U}_{k}}} e^{-r s}\left(A^{\varepsilon}-A\right) u\left(Z^{\varepsilon}(s)\right) d s\right] \rightarrow 0, \quad \text { as } \varepsilon \downarrow 0 .
$$

In addition, using the continuity of $u$ on compact subsets of $\mathscr{O} \cup \Gamma_{1}$ and the continuity of $f$ on compact subsets of $\mathscr{O} \cup \Gamma_{0}$, together with (1.20) and (2.7) we have by Lebesgue's Dominated Convergence Theorem that

$$
\begin{aligned}
& \mathbb{E}_{\mathbb{Q}}^{z}\left[e^{-r\left(t \wedge \nu \mathscr{U}_{k}\right)} u\left(Z^{\varepsilon}\left(t \wedge \nu \mathscr{U}_{k}\right)\right)\right] \rightarrow \mathbb{E}_{\mathbb{Q}}^{z}\left[e^{-r\left(t \wedge \nu \mathscr{U}_{k}\right)} u\left(Z\left(t \wedge \nu \mathscr{U}_{k}\right)\right)\right], \\
& \mathbb{E}_{\mathbb{Q}}^{z}\left[\int_{0}^{t \wedge \nu \mathscr{U}_{k}} e^{-r s} f\left(Z^{\varepsilon}(s)\right) d s\right] \rightarrow \mathbb{E}_{\mathbb{Q}}^{z}\left[\int_{0}^{t \wedge \nu \mathscr{U}_{k}} e^{-r s} f(Z(s)) d s\right],
\end{aligned}
$$

as $\varepsilon \downarrow 0$. Therefore, using (3.10), (3.12) and the preceding limits, we find that (3.9) gives

$$
\mathbb{E}_{\mathbb{Q}}^{z}\left[e^{-r\left(t \wedge \nu \mathscr{U}_{k}\right)} u\left(Z\left(t \wedge \nu \mathscr{U}_{k}\right)\right)\right]=u(z)-\mathbb{E}_{\mathbb{Q}}^{z}\left[\int_{0}^{t \wedge \nu \mathscr{U}_{k}} e^{-r s} f(Z(s)) d s\right] .
$$

Note that by letting $k$ and $t$ tend to $\infty$, we have

$$
t \wedge \nu_{\mathscr{U}_{k}} \rightarrow \nu_{\mathscr{O}}, \quad \text { a.s. }
$$

By using the same argument as that used in the proof of Theorem 1.3 to take the limit as $k$ and $t$ tend to $\infty$ in (3.3), we can take the limit as $k$ and $t$ tend to $\infty$ in (3.14) to give

$$
\mathbb{E}_{\mathbb{Q}}^{z}\left[e^{-r \nu_{\mathscr{O}}} g\left(Z\left(\nu_{\mathscr{O}}\right)\right)\right]=u(z)-\mathbb{E}_{\mathbb{Q}}^{z}\left[\int_{0}^{\nu_{\mathscr{O}}} e^{-r s} f(Z(s)) d s\right] .
$$

This establishes $u=u^{*}$, where $u^{*}$ is given by (1.25), and completes the proof.

Next, we prove the existence of solutions to problem (3.2) when the boundary data $g$ is continuous on suitable portions of the boundary of $\mathscr{O}$.

Proof of Theorem 1.8. Following the comments preceding problem (3.2), we need to show that $u^{*}$, given by (1.23), is a solution to problem (3.2), that $u^{*} \in$ $C_{\text {loc }}\left(\mathscr{O} \cup \partial_{\beta} \mathscr{O}\right) \cap C^{2+\alpha}(\mathscr{O})$, and that $u^{*}$ satisfies the growth assumption (1.20).

Notice that Lemma 2.12, applied with $\theta_{1}=\tau_{\mathscr{O}}, \theta_{2}=\infty$ and $\psi \equiv 0$, shows that $u^{*}$ defined by (1.23) satisfies the growth assumption (1.20). It remains to prove that $u^{*} \in C_{\text {loc }}\left(\mathscr{O} \cup \partial_{\beta} \mathscr{O}\right) \cap C^{2+\alpha}(\mathscr{O})$. Notice that Theorem 1.3 implies that $u^{*}$ is the unique solution to the elliptic boundary value problem (3.2), since any $C_{\text {loc }}\left(\mathscr{O} \cup \partial_{\beta} \mathscr{O}\right) \cap C^{2}(\mathscr{O})$ solution must coincide with $u^{*}$. 
By hypothesis and the definition of $\partial_{\beta} \mathscr{O}$ in (3.1), we have $g \in C_{\text {loc }}\left(\overline{\partial_{\beta} \mathscr{O}}\right)$. Since $\overline{\partial_{\beta} \mathscr{O}}$ is closed, we may use [21, Theorem 3.1.2] to extend $g$ to $\mathbb{R}^{2}$ such that its extension $\tilde{g} \in C_{\text {loc }}\left(\mathbb{R}^{2}\right)$. We organize the proof in two steps.

Step $1\left(u^{*} \in C^{2+\alpha}(\mathscr{O})\right)$. Let $\left\{\mathscr{O}_{k}: k \in \mathbb{N}\right\}$ be an increasing sequence of $C^{2+\alpha}$ subdomains of $\mathscr{O}$ as in the proof of Theorem 1.3. We notice that on each domain $\mathscr{O}_{k}$ the differential operator $A$ is uniformly elliptic with $C^{\infty}\left(\overline{\mathscr{O}}_{k}\right)$ coefficients. From our hypotheses, we have $f \in C^{\alpha}\left(\overline{\mathscr{O}}_{k}\right)$ and $\tilde{g} \in C\left(\overline{\mathscr{O}}_{k}\right)$. Therefore, [25, Theorem 6.13] implies that the elliptic boundary value problem,

$$
\begin{cases}A u=f & \text { on } \mathscr{O}_{k}, \\ u=\tilde{g} & \text { on } \partial \mathscr{O}_{k},\end{cases}
$$

admits a unique solution $u_{k} \in C\left(\overline{\mathscr{O}}_{k}\right) \cap C^{2+\alpha}\left(\mathscr{O}_{k}\right)$. Moreover, by ${ }^{3}[24$, Theorem 6.5.1], $u_{k}$ admits a stochastic representation on $\overline{\mathscr{O}}_{k}$,

$$
u_{k}(z)=\mathbb{E}_{\mathbb{Q}}^{z}\left[e^{-r \tau_{\mathscr{O}_{k}}} \tilde{g}\left(Z\left(\tau_{\mathscr{O}_{k}}\right)\right) 1_{\left\{\tau_{\mathscr{\sigma}_{k}}<\infty\right\}}\right]+\mathbb{E}_{\mathbb{Q}}^{z}\left[\int_{0}^{\tau_{\mathscr{O}_{k}}} e^{-r s} f(Z(s)) d s\right]
$$

Our goal is to show that $u_{k}$ converges pointwise to $u^{*}$ on $\mathscr{O}$. Recall that $\tau_{k}$ is an increasing sequence of stopping times which converges to $\tau_{\mathscr{O}}$ almost surely. Using $\tilde{g} \in C_{\text {loc }}\left(\mathscr{O} \cup \partial_{\beta} \mathscr{O}\right)$ and the continuity of the sample paths of the Heston process, the growth estimate (1.20) and Lemma 2.12, the same argument used in the proof of Theorem 1.3 shows that the sequence $\left\{u_{k}: k \in \mathbb{N}\right\}$ converges pointwise to $u^{*}$ on $\mathscr{O}$.

Fix $z_{0}:=\left(x_{0}, y_{0}\right) \in \mathscr{O}$, and choose a Euclidean ball $B:=B\left(z_{0}, r_{0}\right)$ such that $\bar{B} \subset \mathscr{O}$. We denote $B_{1 / 2}=B\left(z_{0}, r_{0} / 2\right)$. As in the proof of Lemma 2.12 the sequence $u_{k}$ is uniformly bounded on $\bar{B}$ because it obeys

$$
\left|u_{k}(z)\right| \leq \bar{C}\left(1+e^{M_{1} y}+e^{M_{2} x}\right), \quad \forall z=(x, y) \in B, \quad \forall k \in \mathbb{N} .
$$

From the interior Schauder estimates [25, Corollary 6.3], the sequence $\left\{u_{k}: k \in \mathbb{N}\right\}$ has uniformly bounded $C^{2+\alpha}\left(\bar{B}_{1 / 2}\right)$ norms. The compactness of the embedding $C^{2+\alpha}\left(\bar{B}_{1 / 2}\right) \hookrightarrow C^{2+\gamma}\left(\bar{B}_{1 / 2}\right)$, for $0 \leq \gamma<\alpha$, shows that, after passing to a subsequence, the sequence $\left\{u_{k}: k \in \mathbb{N}\right\}$ converges in $C^{2+\gamma}\left(\bar{B}_{1 / 2}\right)$ to $u^{*} \in C^{2+\gamma}\left(\bar{B}_{1 / 2}\right)$, and so $A u^{*}=f$ on $\bar{B}_{1 / 2}$. Because the subsequence has uniformly bounded $C^{2+\alpha}\left(\bar{B}_{1 / 2}\right)$ norms and it converges strongly in $C^{2}\left(\bar{B}_{1 / 2}\right)$ to $u^{*}$, we obtain that $u^{*} \in C^{2+\alpha}\left(\bar{B}_{1 / 2}\right)$.

Step $2\left(u^{*} \in C_{\text {loc }}\left(\mathscr{O} \cup \partial_{\beta} \mathscr{O}\right)\right)$. From the previous step, we know that $u^{*} \in C(\mathscr{O})$, so it remains to show continuity of $u^{*}$ up to $\partial_{\beta} \mathscr{O}$. We consider two cases.

Case $1\left(u^{*} \in C_{\text {loc }}\left(\mathscr{O} \cup \Gamma_{1}\right)\right.$, for all $\left.\beta>0\right)$. First, we show that $u^{*}$ is continuous up to $\Gamma_{1}$. We fix $z_{0} \in \Gamma_{1}$, and let $B$ be an open ball centered at $z_{0}$, such that $\bar{B} \cap \partial \mathbb{H}=\varnothing$. Let $U:=B \cap \mathscr{O}$. Let the function $\hat{g}$ be defined on $\partial U$ such that it coincides with $g$ on $\partial U \cap \partial \mathscr{O}$ and it coincides with $u^{*}$ on $\partial U \cap \mathscr{O}$.

Claim 3.1. The strong Markov property of the Heston process $(Z(s))_{s \geq 0}$ and the definition (1.23) of $u^{*}$ imply that

$$
u^{*}(z)=\mathbb{E}_{\mathbb{Q}}^{z}\left[e^{-r \tau_{U}} \hat{g}\left(Z\left(\tau_{U}\right)\right)\right]+\mathbb{E}_{\mathbb{Q}}^{z}\left[\int_{0}^{\tau_{U}} e^{-r t} f(Z(t)) d t\right], \quad \forall z \in U .
$$

\footnotetext{
${ }^{3}$ See also [30, Proposition 5.7.2] and [38, Theorem 9.1.1 \& Corollary 9.1.2].
} 
Proof. By Corollary 2.8, the Heston stochastic differential equation (1.17) admits a unique strong solution, for any initial point $(t, x, y) \in[0, \infty) \times \mathbb{R} \times[0, \infty)$, and [16. Theorem 1.16(c)] shows that the solution satisfies the strong Markov property.

Let $z \in U$; then $\tau_{U}^{z} \leq \tau_{\mathscr{O}}^{z}$ a.s. Since $Z$ is a time-homogeneous strong Markov process, we obtain

$$
\begin{aligned}
\mathbb{E}_{\mathbb{Q}}^{z}\left[e^{-r \tau_{\mathscr{O}}} g\left(Z\left(\tau_{\mathscr{O}}\right)\right)\right] & =\mathbb{E}_{\mathbb{Q}}^{z}\left[\mathbb{E}_{\mathbb{Q}}^{z}\left[e^{-r \tau_{\mathscr{O}}} g\left(Z\left(\tau_{\mathscr{O}}\right)\right)\right] \mid \mathscr{F}\left(\tau_{U}\right)\right] \\
& =\mathbb{E}_{\mathbb{Q}}^{z}\left[e^{-r \tau_{U}} \mathbb{E}_{\mathbb{Q}}^{Z\left(\tau_{U}\right)}\left[e^{-r \tau_{\mathscr{O}}} g\left(Z\left(\tau_{\mathscr{O}}\right)\right)\right]\right],
\end{aligned}
$$

which can be written as

$$
\begin{aligned}
\mathbb{E}_{\mathbb{Q}}^{z}\left[e^{-r \tau_{\mathscr{O}}} g\left(Z\left(\tau_{\mathscr{O}}\right)\right)\right]= & \mathbb{E}_{\mathbb{Q}}^{z}\left[e^{-r \tau_{U}} g\left(Z\left(\tau_{U}\right)\right) \mathbf{1}_{\left\{\tau_{U}=\tau_{\mathscr{O}}\right\}}\right] \\
& +\mathbb{E}_{\mathbb{Q}}^{z}\left[e^{-r \tau_{U}} \mathbb{E}_{\mathbb{Q}}^{Z\left(\tau_{U}\right)}\left[e^{-r \tau_{\mathscr{O}}} g\left(Z\left(\tau_{\mathscr{O}}\right)\right)\right] \mathbf{1}_{\left\{\tau_{U}<\tau_{\mathscr{O}}\right\}}\right] .
\end{aligned}
$$

Similarly, we have for the integral term

$$
\begin{aligned}
\mathbb{E}_{\mathbb{Q}}^{z}\left[\int_{0}^{\tau_{\mathscr{C}}} e^{-r t} f(Z(t)) d t\right]= & \mathbb{E}_{\mathbb{Q}}^{z}\left[\int_{0}^{\tau_{U}} e^{-r t} f(Z(t)) d t\right] \\
& +\mathbb{E}_{\mathbb{Q}}^{z}\left[\mathbf{1}_{\left\{\tau_{U}<\tau_{\mathscr{O}}\right\}} \int_{\tau_{U}}^{\tau_{\mathscr{O}}} e^{-r t} f(Z(t)) d t\right],
\end{aligned}
$$

and so, by conditioning the second term in the preceding equality on $\mathscr{F}\left(\tau_{U}\right)$ and applying the strong Markov property, we have

$$
\begin{aligned}
\mathbb{E}_{\mathbb{Q}}^{z}\left[\mathbf{1}_{\left\{\tau_{U}<\tau_{\mathscr{O}}\right\}} \int_{\tau_{U}}^{\tau_{\mathscr{O}}} e^{-r t} f(Z(t)) d t\right] \\
\quad=\mathbb{E}_{\mathbb{Q}}^{z}\left[\mathbb{E}_{\mathbb{Q}}^{z}\left[\mathbf{1}_{\left\{\tau_{U} \leq \tau_{\mathscr{O}}\right\}} \int_{\tau_{U}}^{\tau_{\mathscr{O}}} e^{-r t} f(Z(t)) d t\right] \mid \mathscr{F}\left(\tau_{U}\right)\right] \\
\quad=\mathbb{E}_{\mathbb{Q}}^{z}\left[\mathbf{1}_{\left\{\tau_{U}<\tau_{\mathscr{O}}\right\}} e^{-r \tau_{U}} \mathbb{E}_{\mathbb{Q}}^{Z\left(\tau_{U}\right)}\left[\int_{0}^{\tau_{\mathscr{O}}} e^{-r t} f(Z(t)) d t\right]\right] .
\end{aligned}
$$

Combining (3.19) and (3.20) in (1.23), we obtain

$$
\begin{aligned}
u^{*}(z)= & \mathbb{E}_{\mathbb{Q}}^{z}\left[e^{-r \tau_{U}} g\left(Z\left(\tau_{U}\right)\right) \mathbf{1}_{\left\{\tau_{U}=\tau_{\mathscr{O}}\right\}}\right]+\mathbb{E}_{\mathbb{Q}}^{z}\left[\int_{0}^{\tau_{U}} e^{-r t} f(Z(t)) d t\right] \\
& +\mathbb{E}_{\mathbb{Q}}^{z}\left[e^{-r \tau_{U}} \mathbf{1}_{\left\{\tau_{U}<\tau_{\mathscr{O}}\right\}} \mathbb{E}_{\mathbb{Q}}^{Z\left(\tau_{U}\right)}\left[e^{-r \tau_{\mathscr{O}}} g\left(Z\left(\tau_{\mathscr{O}}\right)\right)+\int_{0}^{\tau_{\mathscr{O}}} e^{-r t} f(Z(t)) d t\right]\right] .
\end{aligned}
$$

Again using (1.23) for $u^{*}\left(Z\left(\tau_{U}\right)\right)$, the preceding equality yields (3.18). This completes the proof of Claim 3.1 .

By [25, Theorem 6.13] and a straightforward extension of [24, Theorem 6.5.1] from domains with $C^{2}$ boundary to domains with regular boundary, as in [12, $\S 6.2 .6 . A]$, the integral term in (3.18) is the solution on $U$ of the uniformly elliptic partial differential equation $A u^{*}=f$ with homogeneous Dirichlet boundary condition, and it is a continuous function up to $\partial U$. Notice that $\partial U$ satisfies the exterior sphere condition and thus $\partial U$ is regular by 5 [12, Theorem 2.4.4] (see [12, Definitions $2.4 .1 \& 6.2 .3]$ for the definition 6 of regular points of $\partial U$ ). The continuity of the

\footnotetext{
${ }^{4}$ See also [30, Proposition 5.7.2] and 38, Theorem 9.1.1 \& Corollary 9.1.2].

${ }^{5}$ See also [30 Proposition 4.2.15 \& Theorem 4.2.19].

${ }^{6}$ See also [30, Definition 4.2.9] and [38, Definition 9.2.8].
} 
non-integral term in (3.18) at $z_{0}$ follows from Corollary B.2. as $\hat{g}$ is continuous at $z_{0}$ by hypotheses.

It remains to show that, when $0<\beta<1$, the solution $u^{*}$ is continuous up to $\bar{\Gamma}_{0}$. Case $2\left(u^{*} \in C_{\text {loc }}\left(\mathscr{O} \cup \bar{\Gamma}_{0}\right)\right.$, for all $\left.0<\beta<1\right)$. Let $z_{0}=\left(x_{0}, 0\right) \in \bar{\Gamma}_{0}$. We denote by $\theta^{z}$ the first time the process started at $z=(x, y) \in \mathscr{O}$ hits $y=0$. Obviously, we have

$$
\tau_{\mathscr{O}}^{z} \leq \theta^{z}=T_{0}^{y} \quad \text { a.s. }
$$

where $T_{0}^{y}$ is given by (2.11). For $\beta \in(0,1)$, it follows from (2.12) and the preceding inequality between stopping times that $\theta^{z}$ converges to 0 , as $y$ goes to 0 , uniformly in $x \in \mathbb{R}$. Therefore, the integral term in (1.23) converges to zero as $z$ tends to $z_{0}$. Next, we want to show that the non-integral term in (1.23) converges to $g\left(z_{0}\right)$. We rewrite that term as

$$
\begin{aligned}
\mathbb{E}_{\mathbb{Q}}^{z}\left[e^{-r \tau_{\mathscr{O}}} g\left(Z\left(\tau_{\mathscr{O}}\right)\right)\right]-g\left(z_{0}\right)= & \mathbb{E}_{\mathscr{Q}}^{z}\left[e^{-r \tau_{\mathscr{O}}}\left(g\left(Z\left(\tau_{\mathscr{O}}\right)\right)-g\left(z_{0}\right)\right)\right] \\
& +g\left(z_{0}\right)\left(1-\mathbb{E}_{\mathbb{Q}}^{z}\left[e^{-r \tau_{\mathscr{O}}}\right]\right) .
\end{aligned}
$$

From the observation that $\tau_{\mathscr{O}}^{z} \leq \theta^{z}$ a.s., we see that

$$
\mathbb{E}_{\mathbb{Q}}^{z}\left[e^{-r \tau_{\mathscr{O}}}\right] \rightarrow 1 \text {, as } z \rightarrow z_{0}
$$

By (3.22), it remains to show that $\mathbb{E}_{\mathbb{Q}}^{z}\left[e^{-r \tau_{\mathscr{O}}}\left(g\left(Z\left(\tau_{\mathscr{O}}\right)\right)-g\left(z_{0}\right)\right)\right]$ converges to zero, as $z \in \mathscr{O}$ converges to $z_{0}$. We fix $\varepsilon>0$ and choose $\delta_{1}>0$ such that

$$
\left|g(z)-g\left(z_{0}\right)\right|<\varepsilon, \quad \forall z \in B\left(z_{0}, \delta_{1}\right) \cap \partial \mathscr{O} .
$$

From [30, Equation (5.3.18) in Problem 5.3.15], there is a positive constant, $C_{1}$, depending on $z_{0}$ and $\delta_{1}$, such that

$$
\sup _{z \in B\left(z_{0}, \delta_{1}\right) \cap \mathscr{O}} \mathbb{E}_{\mathbb{Q}}^{z}\left[\sup _{0 \leq s \leq t}|Z(s)-z|\right] \leq C_{1} \sqrt{t},
$$

from where it follows that

$$
\sup _{z \in B\left(z_{0}, \delta_{1}\right) \cap \mathscr{O}} \mathbb{Q}^{z}\left(\sup _{0 \leq s \leq t}|Z(s)-z|>\delta_{1} / 2\right) \leq \frac{2 C_{1} \sqrt{t}}{\delta_{1}} .
$$

Next, we choose $t>0$ sufficiently small such that

$$
\frac{2 C_{1} \sqrt{t}}{\delta_{1}}<\varepsilon
$$

and, by (3.21) and (2.12), we may choose $\delta_{2}>0$ sufficiently small such that

$$
\mathbb{Q}\left(T_{0}^{\delta_{2}}>t\right)<\varepsilon
$$

Let $\delta:=\min \left\{\delta_{1} / 2, \delta_{2}\right\}$. We rewrite

$$
\begin{aligned}
e^{-r \tau_{\mathscr{O}}}\left(g\left(Z\left(\tau_{\mathscr{O}}\right)\right)-g\left(z_{0}\right)\right)= & e^{-r \tau_{\mathscr{O}}}\left(g\left(Z\left(\tau_{\mathscr{O}}\right)\right)-g\left(z_{0}\right)\right) \mathbf{1}_{\left\{\tau_{\mathscr{O}} \leq t\right\}} \\
& +e^{-r \tau_{\mathscr{O}}}\left(g\left(Z\left(\tau_{\mathscr{O}}\right)\right)-g\left(z_{0}\right)\right) \mathbf{1}_{\left\{\tau_{\mathscr{O}}>t\right\}}
\end{aligned}
$$

to give

$$
\begin{aligned}
e^{-r \tau_{\mathscr{O}}}( & \left.g\left(Z\left(\tau_{\mathscr{O}}\right)\right)-g\left(z_{0}\right)\right) \\
= & e^{-r \tau_{\mathscr{O}}}\left(g\left(Z\left(\tau_{\mathscr{O}}\right)\right)-g\left(z_{0}\right)\right) \mathbf{1}_{\left\{\tau_{\mathscr{O}} \leq t, \sup _{0 \leq s \leq t}|Z(s)-z|<\delta_{1} / 2\right\}} \\
& +e^{-r \tau_{\mathscr{O}}}\left(g\left(Z\left(\tau_{\mathscr{O}}\right)\right)-g\left(z_{0}\right)\right) \mathbf{1}_{\left\{\tau_{\mathscr{O}} \leq t, \sup _{0 \leq s \leq t}|Z(s)-z| \geq \delta_{1} / 2\right\}} \\
& +e^{-r \tau_{\mathscr{O}}}\left(g\left(Z\left(\tau_{\mathscr{O}}\right)\right)-g\left(z_{0}\right)\right) \mathbf{1}_{\left\{\tau_{\mathscr{O}}>t\right\}} .
\end{aligned}
$$


By (3.24), we have for all $z \in B\left(z_{0}, \delta\right) \cap \mathscr{O}$,

$$
\mathbb{E}_{\mathbb{Q}}^{z}\left[\left|g\left(Z\left(\tau_{\mathscr{O}}\right)\right)-g\left(z_{0}\right)\right| \mathbf{1}_{\left\{\tau_{\mathscr{O}} \leq t, \sup _{0 \leq s \leq t}|Z(s)-z|<\delta_{1} / 2\right\}}\right]<\varepsilon .
$$

We choose $p>1$ such that $p M_{1}<\mu$ and $p M_{2}<1$. Notice that this is possible because we assumed the coefficients $M_{1}<\mu$ and $M_{2}<1$. Then, from the growth estimate (1.20) for $g$, we have

$$
\left|e^{-r \tau_{\mathscr{C}}} g\left(Z\left(\tau_{\mathscr{O}}\right)\right)\right|^{p} \leq C e^{-r p \tau_{\mathscr{O}}}\left(1+e^{p M_{1} Y\left(\tau_{\mathscr{O}}\right)}+e^{p M_{2} X\left(\tau_{\mathscr{O}}\right)}\right) .
$$

From property (2.14) applied with $c=p M_{1}<\mu$ and property (2.13) applied with $c=p M_{2} \in(0,1)$, we use the condition $M_{1} \leq r /(\kappa \vartheta)$ to obtain

$$
\mathbb{E}_{\mathbb{Q}}^{z}\left[\left|e^{-r \tau_{\mathscr{O}}} g\left(Z\left(\tau_{\mathscr{O}}\right)\right)\right|^{p}\right] \leq C\left(1+e^{p M_{1} y}+e^{p M_{2} x}\right) .
$$

Let $C_{2}>0$ be a bound on the right-hand side of the preceding inequality, for all $z=(x, y) \in B\left(z_{0}, \delta\right) \cap \mathscr{O}$. By the Cauchy-Schwarz inequality, we have

$$
\begin{aligned}
\mid \mathbb{E}_{\mathbb{Q}}^{z} & {\left[e^{-r \tau_{\mathscr{O}}}\left(g\left(Z\left(\tau_{\mathscr{O}}\right)\right)-g\left(z_{0}\right)\right) \mathbf{1}_{\left\{\tau_{\mathscr{O}}>t\right\}}\right] \mid } \\
& \leq \mathbb{E}_{\mathscr{Q}}^{z}\left[e^{-r p \tau_{\mathscr{O}}}\left|g\left(Z\left(\tau_{\mathscr{O}}\right)\right)-g\left(z_{0}\right)\right|^{p}\right]^{1 / p} \mathbb{Q}^{z}\left(\tau_{\mathscr{O}}>t\right)^{1 / p^{\prime}}
\end{aligned}
$$

where $p^{\prime}>1$ denotes the conjugate exponent of $p$. Using the fact that $\tau_{\mathscr{O}}^{z} \leq T_{0}^{\delta_{2}}$ from (3.21) and (3.27), we obtain in the preceding inequality

$$
\begin{aligned}
\mid \mathbb{E}_{\mathbb{Q}}^{z} & {\left[e^{-r \tau_{\mathscr{O}}}\left(g\left(Z\left(\tau_{\mathscr{O}}\right)\right)-g\left(z_{0}\right)\right) \mathbf{1}_{\left\{\tau_{\mathscr{O}}>t\right\}}\right] \mid } \\
& \leq 2 C_{2}^{1 / p} \mathbb{Q}^{z}\left(T_{0}>t\right)^{1 / p^{\prime}} \\
& \leq 2 C_{2}^{1 / p} \varepsilon^{1 / p^{\prime}}, \quad \forall z \in B\left(z_{0}, \delta\right) \cap \mathscr{O} .
\end{aligned}
$$

From the inequality

$$
\begin{aligned}
& \mid \mathbb{E}_{\mathbb{Q}}^{z} {\left[e^{-r \tau_{\mathscr{O}}}\left(g\left(Z\left(\tau_{\mathscr{O}}\right)\right)-g\left(z_{0}\right)\right) \mathbf{1}_{\left\{\tau_{\mathscr{O}} \leq t, \sup _{0 \leq s \leq t}|Z(s)-z| \geq \delta_{1} / 2\right\}}\right] \mid } \\
& \quad \leq \mathbb{E}_{\mathbb{Q}}^{z}\left[e^{-r p \tau_{\mathscr{O}}}\left|g\left(Z\left(\tau_{\mathscr{O}}\right)\right)-g\left(z_{0}\right)\right|^{p}\right]^{1 / p} \mathbb{Q}^{z}\left(\sup _{0 \leq s \leq t}|Z(s)-z| \geq \delta_{1} / 2\right)^{1 / p^{\prime}}
\end{aligned}
$$

inequalities (3.25) and (3.26) and the definition of $C_{2}$ yield

$$
\left|\mathbb{E}_{\mathbb{Q}}^{z}\left[e^{-r \tau_{\mathscr{O}}}\left(g\left(Z\left(\tau_{\mathscr{O}}\right)\right)-g\left(z_{0}\right)\right) \mathbf{1}_{\left\{\tau_{\mathscr{O}} \leq t, \sup _{0 \leq s \leq t}|Z(s)-z| \geq \delta_{1} / 2\right\}}\right]\right| \leq 2 C_{2}^{1 / p} \varepsilon^{1 / p^{\prime}}
$$

Substituting (3.29), (3.30), and (3.31) in (3.28), we obtain

$$
\mathbb{E}\left[e^{-r \tau_{\mathscr{O}}}\left(g\left(Z\left(\tau_{\mathscr{O}}\right)\right)-g\left(z_{0}\right)\right)\right]<\left(1+4 C_{2}^{1 / p}\right)\left(\varepsilon+\varepsilon^{1 / p^{\prime}}\right), \quad \forall z \in B\left(z_{0}, \delta\right) \cap \mathscr{O},
$$

and so $u^{*}$ is continuous up to $\bar{\Gamma}_{0}$, when $0<\beta<1$.

This concludes the proof that $u^{*} \in C_{\text {loc }}\left(\mathscr{O} \cup \partial_{\beta} \mathscr{O}\right)$, for all $\beta>0$.

This completes the proof of Theorem 1.8 .

We now prove the existence of solutions to problem (3.2) when the boundary data $g$ is Hölder continuous on suitable portions of the boundary of $\mathscr{O}$.

Proof of Theorem 1.9. The proof of the theorem is the same as that of Theorem 1.8. with the exception that Case 1 of Step 2 can be simplified by applying the classical boundary Schauder estimates. Also, instead of using the sequence of subdomains $\left\{\mathscr{O}_{k}: k \in \mathbb{N}\right\}$ precompactly contained in $\mathscr{O}$, as in the proof of Theorem 1.3, we 
consider an increasing sequence, $\left\{\mathscr{D}_{k}: k \in \mathbb{N}\right\}$, of $C^{2+\alpha}$ subdomains of $\mathscr{O}$ (see [25, Definition $\S 6.2]$ ) such that each $\mathscr{D}_{k}$ satisfies

$$
\mathscr{O} \cap(-k, k) \times(1 / k, k) \subset \mathscr{D}_{k} \subset \mathscr{O} \cap(-2 k, 2 k) \times(1 /(2 k), 2 k), \quad \forall k \in \mathbb{N},
$$

and

$$
\bigcup_{k \in \mathbb{N}} \mathscr{D}_{k}=\mathscr{O}
$$

Since $\Gamma_{1}$ is assumed to be of class $C^{2+\alpha}$, we may choose $\mathscr{D}_{k}$ to be of class $C^{2+\alpha}$.

Let $z_{0} \in \Gamma_{1}$ and let $r_{0}>0$ be small enough such that $B\left(z_{0}, r_{0}\right) \cap \Gamma_{0}=\varnothing$. Let

$$
D:=B\left(z_{0}, r_{0}\right) \cap \mathscr{O} \text { and } D^{\prime}:=B\left(z_{0}, r_{0} / 2\right) \cap \mathscr{O} \text {. }
$$

By (3.32), we may choose $k_{0} \in \mathbb{N}$ large enough such that $D \subset \mathscr{D}_{k}$, for all $k \geq k_{0}$. Using $f \in C^{\alpha}(\bar{D}), g \in C^{2+\alpha}(\bar{D})$ and applying [25, Corollary 6.7], and the fact that $u_{k}$ solves (3.16), with $g$ replacing $\tilde{g}$ and $\mathscr{D}_{k}$ replacing $\mathscr{O}_{k}$,

$$
\left\|u_{k}\right\|_{C^{2+\alpha}\left(\bar{D}^{\prime}\right)} \leq C\left(\left\|u_{k}\right\|_{C(\bar{D})}+\|\tilde{g}\|_{C^{2+\alpha}(\bar{D})}+\|f\|_{C^{\alpha}(\bar{D})}\right), \quad \forall k \geq k_{0},
$$

where $C>0$ is a constant depending only on the coefficients of $A$ and on the domains $D$ and $D^{\prime}$. Combining the preceding inequality with the uniform bound on the $C(\bar{D})$ norms of the sequence $\left\{u_{k}: k \in \mathbb{N}\right\}$, resulting from Lemma 2.12, the compactness of the embedding of $C^{2+\alpha}\left(\bar{D}^{\prime}\right) \hookrightarrow C^{2+\gamma}\left(\bar{D}^{\prime}\right)$, when $0 \leq \gamma<\alpha$, implies that a subsequence converges strongly to $u^{*}$ in $C^{2+\gamma}\left(\bar{D}^{\prime}\right)$. Therefore, $u^{*} \in C^{2+\gamma}\left(\bar{D}^{\prime}\right)$, and $A u^{*}=f$ on $D^{\prime}$ and $u^{*}=g$ on $\partial D^{\prime} \cap \Gamma_{1}$. Moreover, $u^{*} \in C^{2+\alpha}\left(\bar{D}^{\prime}\right)$, since $u_{k} \in C^{2+\alpha}\left(\bar{D}^{\prime}\right)$, for all $k \geq k_{0}$, and the sequence converges in $C^{2+\gamma}\left(\bar{D}^{\prime}\right)$ to $u^{*}$. Combining the boundary estimate (3.33) with Step 1 in the proof of Theorem 1.8. we obtain the conclusion that $u^{*} \in C_{\text {loc }}^{2+\alpha}\left(\mathscr{O} \cup \Gamma_{1}\right)$.

Remark 3.2 (Validity of the stochastic representation for strong solutions). The stochastic representation (1.25) for solutions to problem (1.1) with boundary condition along $\Gamma_{1}$ is valid if we replace the condition $u \in C_{\mathrm{loc}}\left(\mathscr{O} \cup \Gamma_{1}\right) \cap C_{s, \text { loc }}^{1,1}\left(\mathscr{O} \cup \Gamma_{0}\right)$ in the hypotheses of Theorem 1.5 with the weaker condition that $u \in C_{\mathrm{loc}}\left(\mathscr{O} \cup \Gamma_{1}\right) \cap W_{\mathrm{loc}}^{2,2}(\mathscr{O})$ or $u \in C_{\text {loc }}\left(\mathscr{O} \cup \Gamma_{1}\right) \cap W_{\text {loc }}^{2, p}(\mathscr{O})$, with $p>d / 2+1$ (in this article, $d=2$ ), as long as suitable estimates of the transition probabilities of the Heston process hold (see the proofs of [5, Lemma 2.8.1 and Theorem 2.8.5]). Notice that [5, Theorem 2.8.5] applies to our framework with $p=\infty$, because in this case we do not need any estimates of the transition probabilities of the Heston process.

\section{Elliptic obstacle PRoblem}

This section contains the proofs of Theorems 1.12 and 1.13. As in problem (3.2), the questions of the uniqueness of solutions to problem (1.2) with Dirichlet boundary condition along $\Gamma_{1}$, when $\beta \geq 1$, and along $\partial \mathscr{O}$, when $0<\beta<1$, are similar in nature. We can conveniently treat them together as

$$
\begin{cases}\min \{A u-f, u-\psi\}=0 & \text { on } \mathscr{O}, \\ u=g & \text { on } \partial_{\beta} \mathscr{O},\end{cases}
$$

where $\partial_{\beta} \mathscr{O}$ is given by (3.1).

Proof of Theorem 1.12, Lemma 2.12 indicates that $u^{*}$ given by (1.27) satisfies (1.20), so the growth assumption on $u$ in Theorem 1.12 is justified. 
By the preceding remarks, it suffices to prove that the stochastic representation (1.27) holds for solutions $u \in C_{\text {loc }}\left(\mathscr{O} \cup \partial_{\beta} \mathscr{O}\right) \cap C^{1,1}(\mathscr{O})$ to problem (4.1). We consider the two situations: $u \geq u^{*}$ and $u \leq u^{*}$ on $\mathscr{O} \cup \partial_{\beta} \mathscr{O}$, where $u^{*}$ is defined by (1.27).

Step 1 (Proof that $u \geq u^{*}$ on $\mathscr{O} \cup \partial_{\beta} \mathscr{O}$ ). Let $\left\{\mathscr{O}_{k}: k \in \mathbb{N}\right\}$ be an increasing sequence of $C^{2+\alpha}$ subdomains of $\mathscr{O}$ as in the proof of Theorem 1.3. By using the same argument as that used in the proof of Theorem 1.3 to establish that $u=u^{*}$, and the fact that $u \in C^{1,1}(\mathscr{O})$, Itô's lemma [5. Theorem 2.8.5] with $p=\infty$ yields, for any stopping time $\theta \in \mathscr{T}$,

$$
\mathbb{E}_{\mathbb{Q}}^{z}\left[e^{-r\left(\theta \wedge \tau_{\mathscr{O}_{k}}\right)} u\left(Z\left(\theta \wedge \tau_{\mathscr{O}_{k}}\right)\right)\right]=u(z)-\mathbb{E}_{\mathbb{Q}}^{z}\left[\int_{0}^{\theta \wedge \tau_{\mathscr{O}_{k}}} e^{-r s} A u(Z(s)) d s\right] .
$$

By splitting the right-hand side in the preceding identity,

$$
\begin{aligned}
& \mathbb{E}_{\mathbb{Q}}^{z}\left[e^{-r\left(\theta \wedge \tau_{\mathscr{O}_{k}}\right)} u\left(Z\left(\theta \wedge \tau_{\mathscr{O}_{k}}\right)\right)\right] \\
& \quad=\mathbb{E}_{\mathbb{Q}}^{z}\left[e^{-r \tau_{\mathscr{O}_{k}}} u\left(Z\left(\theta \wedge \tau_{\mathscr{O}_{k}}\right)\right) \mathbf{1}_{\left\{\tau_{\mathscr{O}_{k}} \leq \theta\right\}}\right]+\mathbb{E}_{\mathbb{Q}}^{z}\left[e^{-r \theta} u\left(Z\left(\theta \wedge \tau_{\mathscr{O}_{k}}\right)\right) \mathbf{1}_{\left\{\tau_{\mathscr{O}_{k}}>\theta\right\}}\right],
\end{aligned}
$$

and using the fact that $u \geq \psi$ on $\mathscr{O}$ and $A u \geq f$ a.e. on $\mathscr{O}$, the identity (4.2) gives

$$
\begin{aligned}
u(z) \geq & \mathbb{E}_{\mathbb{Q}}^{z}\left[e^{-r \theta} \psi(Z(\theta)) \mathbf{1}_{\left\{\theta<\tau_{\mathscr{C}_{k}}\right\}}\right] \\
& +\mathbb{E}_{\mathbb{Q}}^{z}\left[e^{-r \tau_{\mathscr{C}_{k}}} u\left(Z\left(\tau_{\mathscr{O}_{k}}\right)\right) \mathbf{1}_{\left\{\tau_{\mathscr{C}_{k}} \leq \theta\right\}}\right]+\mathbb{E}^{z}\left[\int_{0}^{\theta \wedge \tau_{\mathscr{C}_{k}}} e^{-r s} f(Z(s)) d s\right] .
\end{aligned}
$$

Just as in the proof of Theorem 1.3, the collections of random variables

$$
\left\{e^{-r \theta} \psi(Z(\theta)) \mathbf{1}_{\left\{\theta<\tau_{\mathscr{C}_{k}}\right\}}: k \in \mathbb{N}\right\} \text { and }\left\{e^{-r \tau_{\mathscr{O}_{k}}} u\left(Z\left(\tau_{\mathscr{O}_{k}}\right)\right) \mathbf{1}_{\left\{\tau_{\mathscr{O}_{k}} \leq \theta\right\}}: k \in \mathbb{N}\right\}
$$

are uniformly integrable because $u$ and $\psi$ satisfy the pointwise growth estimate (1.20). From the continuity of $u$ and $\psi$ on $\mathscr{O} \cup \partial_{\beta} \mathscr{O}$, we also have the a.s. convergence,

$$
\begin{aligned}
& e^{-r \theta} \psi(Z(\theta)) \mathbf{1}_{\left\{\theta<\tau_{\mathscr{C}_{k}}\right\}} \rightarrow e^{-r \theta} \psi(Z(\theta)) \mathbf{1}_{\left\{\theta<\tau_{\mathscr{O}}\right\}}, \quad \text { as } k \rightarrow \infty, \\
& e^{-r \tau_{\mathscr{O}_{k}}} u\left(Z\left(\tau_{\mathscr{O}_{k}}\right)\right) \mathbf{1}_{\left\{\tau_{\mathscr{O}_{k}} \leq \theta\right\}} \rightarrow e^{-r \tau_{\mathscr{O}}} u\left(Z\left(\tau_{\mathscr{O}}\right)\right) \mathbf{1}_{\left\{\tau_{\mathscr{O}} \leq \theta\right\}}, \quad \text { as } k \rightarrow \infty .
\end{aligned}
$$

Therefore, by [6, Theorem 16.13], we can take the limit as $k$ tends to $\infty$ in inequality (4.3) to obtain, for all $\theta \in \mathscr{T}$,

$$
\begin{aligned}
u(z) \geq & \mathbb{E}_{\mathbb{Q}}^{z}\left[e^{-r \theta} \psi(Z(\theta)) \mathbf{1}_{\left\{\theta<\tau_{\mathscr{O}}\right\}}\right]+\mathbb{E}_{\mathbb{Q}}^{z}\left[e^{-r \tau_{\mathscr{O}}} u\left(Z\left(\tau_{\mathscr{O}}\right)\right) \mathbf{1}_{\left\{\tau_{\mathscr{O}} \leq \theta\right\}}\right] \\
& +\mathbb{E}_{\mathbb{Q}}^{z}\left[\int_{0}^{\theta \wedge \tau_{\mathscr{O}}} e^{-r s} f(Z(s)) d s\right],
\end{aligned}
$$

which yields $u \geq u^{*}$ on $\mathscr{O} \cup \partial_{\beta} \mathscr{O}$.

Step 2 (Proof that $u \leq u^{*}$ on $\mathscr{O} \cup \partial_{\beta} \mathscr{O}$ ). The continuation region,

$$
\mathscr{C}:=\{u>\psi\},
$$

is an open set by the continuity of $u$ and $\psi$. We denote the first exit time of $Z^{t, z}$ from the continuation region, $\mathscr{C}$, by

$$
\tilde{\tau}^{t, z}:=\left\{s \geq t: Z^{t, z}(s) \notin \mathscr{C}\right\},
$$

and write $\tilde{\tau}=\tilde{\tau}^{t, z}$ for brevity. This is indeed a stopping time because the process $Z^{t, z}$ is continuous and $\mathscr{C}$ is open. By the same argument used in Step 1 with $\theta$ 
replaced by $\tilde{\tau}$, we obtain that all inequalities hold with equalities because $u(Z(\tilde{\tau}))=$ $\psi(Z(\tilde{\tau}))$ and $A u=f$ on the continuation region, $\mathscr{C}$. Therefore,

$$
\begin{aligned}
u(z)= & \mathbb{E}_{\mathbb{Q}}^{z}\left[e^{-r \tilde{\tau}} \psi(Z(\tilde{\tau})) \mathbf{1}_{\left\{\tilde{\tau}<\tau_{\mathscr{O}}\right\}}\right]+\mathbb{E}_{\mathbb{Q}}^{z}\left[e^{-r \tau_{\mathscr{O}}} g\left(Z\left(\tau_{\mathscr{O}}\right)\right) \mathbf{1}_{\left\{\tau_{\mathscr{O}} \leq \tilde{\tau}_{\mathscr{O}}\right\}}\right] \\
& +\mathbb{E}_{\mathbb{Q}}^{z}\left[\int_{0}^{\tilde{\tau} \wedge \tau_{\mathscr{O}}} e^{-r s} f(Z(s)) d s\right]
\end{aligned}
$$

which implies that $u \leq u^{*}$.

By combining the preceding two steps, we obtain the stochastic representation (1.27) of solutions to problem (4.1), and hence the uniqueness assertion.

Proof of Theorem 1.13. Lemma 2.12 indicates that $u^{*}$ given by (1.28) satisfies (1.20), so the growth assumption on $u$ in Theorem 1.12 is justified.

Our goal is to show that if $0<\beta<1$ and $u \in C_{\text {loc }}\left(\mathscr{O} \cup \Gamma_{1}\right) \cap C_{s, \text { loc }}^{1,1}\left(\mathscr{O} \cup \Gamma_{0}\right)$ is a solution to problem (1.2) with Dirichlet boundary condition (1.4) along $\Gamma_{1}$ and satisfying the growth estimate (1.20), then it admits the stochastic representation (1.28). As in the proof of Theorem [1.12, we consider the following two cases.

Step 1 (Proof that $u \geq u^{*}$ on $\mathscr{O} \cup \Gamma_{1}$ ). Let $\varepsilon>0$ and $\left\{\mathscr{U}_{k}: k \in \mathbb{N}\right\}$ be the collection of increasing subdomains as in (3.5). By applying Itô's lemma, we obtain, for all $t>0$ and $\theta \in \mathscr{T}$,

$$
\begin{aligned}
u(x, y+\varepsilon)= & \mathbb{E}_{\mathbb{Q}}^{z}\left[e^{-r\left(t \wedge \nu \mathscr{U}_{k} \wedge \theta\right)} u\left(Z^{\varepsilon}\left(t \wedge \nu \mathscr{U}_{k} \wedge \theta\right)\right)\right] \\
& +\mathbb{E}_{\mathbb{Q}}^{z}\left[\int_{0}^{t \wedge \nu \mathscr{U}_{k} \wedge \theta} e^{-r s} A^{\varepsilon} u\left(Z^{\varepsilon}(s)\right) d s\right],
\end{aligned}
$$

where $\nu_{\mathscr{U}_{k}}$ is given by (1.22) and $Z^{\varepsilon}$ is defined in (3.6), and $A^{\varepsilon}$ is defined by (3.8). By (1.2) and (3.8), the preceding identity gives

$$
\begin{aligned}
u(x, y+\varepsilon) \geq & \mathbb{E}_{\mathbb{Q}}^{z}\left[e^{-r\left(t \wedge \nu \mathscr{U}_{k} \wedge \theta\right)} u\left(Z^{\varepsilon}\left(t \wedge \nu \mathscr{U}_{k} \wedge \theta\right)\right)\right] \\
& +\mathbb{E}_{\mathbb{Q}}^{z}\left[\int_{0}^{t \wedge \nu \mathscr{U}_{k} \wedge \theta} e^{-r s} f\left(Z^{\varepsilon}(s)\right) d s\right] \\
& +\mathbb{E}_{\mathbb{Q}}^{z}\left[\int_{0}^{t \wedge \nu \mathscr{U}_{k} \wedge \theta} e^{-r s}\left(A^{\varepsilon}-A\right) u\left(Z^{\varepsilon}(s)\right) d s\right] .
\end{aligned}
$$

First, we take the limit as $\varepsilon$ tends to 0 in (4.7). We can assume without loss of generality that $\varepsilon<1 / k$, for any fixed $k \in \mathbb{N}$. The residual term $\left(A^{\varepsilon}-A\right) u$ then obeys estimate (3.11) because $u \in C_{s, \text { loc }}^{1,1}\left(\mathscr{O} \cup \Gamma_{0}\right)$. Therefore, (3.12) also holds in the present case. In addition, using the continuity of $f, u, D u$ and $y D^{2} u$ on compact subsets of $\mathscr{O} \cup \Gamma_{0}$, we see that (3.13) holds, and so, by taking the limit as $\varepsilon \downarrow 0$ in (4.7), together with (1.20) and (2.7)

$$
\begin{aligned}
u(z) \geq & \mathbb{E}_{\mathbb{Q}}^{z}\left[e^{-r\left(t \wedge \nu \mathscr{U}_{k} \wedge \theta\right)} u\left(Z\left(t \wedge \nu \mathscr{U}_{k} \wedge \theta\right)\right)\right] \\
& +\mathbb{E}_{\mathbb{Q}}^{z}\left[\int_{0}^{t \wedge \nu \mathscr{U}_{k} \wedge \theta} e^{-r s} f(Z(s)) d s\right] .
\end{aligned}
$$


Finally, letting $k$ and $t$ tend to $\infty$ and using the convergence (3.15), the same argument employed in the proof of Theorem 1.3 can be applied to conclude that $u \geq u^{*}$ on $\mathscr{O} \cup \Gamma_{1}$, where $u^{*}$ is given by (1.28).

Step 2 (Proof that $u \leq u^{*}$ on $\mathscr{O} \cup \Gamma_{1}$ ). We choose $\theta=\tilde{\tau}$ in the preceding step, where $\tilde{\tau}$ is defined by (4.5). By the definition (4.4) of the continuation region, $\mathscr{C}$, and the obstacle problem (1.2), we notice that inequalities (4.7) and (4.8) hold with equality, and so it follows as in Step 1 that $u \leq u^{*}$ on $\mathscr{O} \cup \Gamma_{1}$.

This completes the proof.

Remark 4.1 (Validity of the stochastic representation for strong solutions). The stochastic representation (1.27) of solutions to problem (4.1), when $\beta>0$, holds under the weaker assumption that $u \in C_{\text {loc }}\left(\mathscr{O} \cup \partial_{\beta} \mathscr{O}\right) \cap W_{\text {loc }}^{2,2}(\mathscr{O})$ or $u \in$ $C_{\text {loc }}\left(\mathscr{O} \cup \partial_{\beta} \mathscr{O}\right) \cap W_{\text {loc }}^{2, p}(\mathscr{O})$, with $p>d / 2+1$ (in this article, $d=2$ ), as long as suitable estimates of the transition probabilities of the Heston process hold (see the proofs of [5, Lemma 2.8.1 and Theorem 2.8.5]). The same observation applies to the stochastic representation (1.28) of solutions to problem (1.2) with Dirichlet boundary condition (1.3) along $\Gamma_{1}$, when $0<\beta<1$.

\section{Parabolic terminal/Boundary value problem}

This section contains the proofs of Theorems 1.16 and 1.18 and of the existence result in Theorem 1.22 Because the Heston process satisfies the strong Markov property, it suffices to prove the stochastic representation of solutions to the terminal value problem for $T$ as small as we like. In particular, without loss of generality, we can choose $T$ such that

Hypothesis 5.1. There is a constant $p_{0}>1$ such that

(1) Condition (2.20) in Lemma 2.14 is satisfied for $p:=p_{0} M_{2}$, where $M_{2} \in$ $[0,1]$ is the constant appearing in (1.29).

(2) One has $p_{0} M_{1} \leq \mu$, where $M_{1} \in[0, \mu)$ in (1.29).

As in 33 we first prove the uniqueness of solutions to the parabolic terminal/boundary value problems (1.7) with different possible Dirichlet boundary conditions depending on the parameter $\beta$. The proofs are similar to those of Theorems 1.3 and 1.5 .

The existence and uniqueness of solutions to problem (1.7) with boundary condition (1.9), when $\beta \geq 1$, and with boundary condition (1.12), when $0<\beta<1$, are similar in nature. By analogy with our treatment of problem (3.2), we define

$$
\varlimsup_{\beta} Q:= \begin{cases}ð^{1} Q & \text { if } \beta \geq 1, \\ ð Q & \text { if } 0<\beta<1,\end{cases}
$$

where we recall that $Q:=(0, T) \times \mathscr{O}$. The preceding problems can then be formulated as

$$
\begin{aligned}
-u_{t}+A u=f & & \text { on } Q, \\
u=g & & \text { on } \partial_{\beta} Q .
\end{aligned}
$$


We now have the

Proof of Theorem 1.16. We choose $T>0$ small enough and $p_{0}>1$ as in Hypothesis 5.1. The pattern of the proof is the same as that of Theorem 1.3. For completeness, we outline the main steps of the argument.

We need to show that if $u \in C_{\mathrm{loc}}\left(Q \cup{ }_{\beta} Q\right) \cap C^{1,1}(Q)$ is a solution to problem (15.2), satisfying the growth bound (1.29), then it admits the stochastic representation (1.30). We choose a collection of increasing subdomains, $\left\{\mathscr{O}_{k}: k \in \mathbb{N}\right\}$, as in the proof of Theorem [1.3. By applying Itô's lemma [5, Theorem 2.8.5] with $p=\infty$, we obtain, for all $t>0$ and $k \in \mathbb{N}$,

$$
\begin{aligned}
\mathbb{E}_{\mathbb{Q}}^{t, z} & {\left[e^{-r\left(\tau_{\mathscr{O}_{k}} \wedge T-t\right)} u\left(\tau_{\mathscr{O}_{k}} \wedge T, Z\left(\tau_{\mathscr{O}_{k}} \wedge T\right)\right)\right] } \\
& =u(t, z)-\mathbb{E}_{\mathbb{Q}}^{t, z}\left[\int_{t}^{\tau_{\mathscr{O}_{k}} \wedge T} e^{-r(s-t)} f(s, Z(s)) d s\right] .
\end{aligned}
$$

We now take the limit as $k$ tends to $\infty$ in the preceding identity. Using (1.29) and Lemma 2.11, we obtain

$$
\begin{aligned}
\mathbb{E}_{\mathbb{Q}}^{t, z} & {\left[\int_{t}^{\tau_{\mathscr{C}_{k}} \wedge T} e^{-r(s-t)} f(s, Z(s)) d s\right] } \\
& \rightarrow \mathbb{E}_{\mathbb{Q}}^{t, z}\left[\int_{t}^{\tau_{\mathscr{O}} \wedge T} e^{-r(s-t)} f(s, Z(s)) d s\right] \text {, as } k \rightarrow \infty .
\end{aligned}
$$

From the continuity of $u$ and of the sample paths of $Z$, we obtain the a.s. convergence as $k$ tends to $\infty$,

$$
e^{-r\left(\tau_{\mathscr{O}_{k}} \wedge T-t\right)} u\left(\tau_{\mathscr{O}_{k}} \wedge T, Z\left(\tau_{\mathscr{O}_{k}} \wedge T\right)\right) \rightarrow e^{-r\left(\tau_{\mathscr{O}} \wedge T\right)} g\left(\tau_{\mathscr{O}} \wedge T, Z\left(\tau_{\mathscr{O}} \wedge T\right)\right) .
$$

In order to prove that, as $k$ tends to $\infty$,

$$
\begin{aligned}
& \mathbb{E}_{\mathbb{Q}}^{t, z} {\left[e^{-r\left(\tau_{\mathscr{O}_{k}} \wedge T-t\right)} u\left(\tau_{\mathscr{O}_{k}} \wedge T, Z\left(\tau_{\mathscr{O}_{k}} \wedge T\right)\right)\right] } \\
& \rightarrow \mathbb{E}_{\mathbb{Q}}^{t, z}\left[e^{-r\left(\tau_{\mathscr{O}} \wedge T\right)} g\left(\tau_{\mathscr{O}} \wedge T, Z\left(\tau_{\mathscr{O}} \wedge T\right)\right)\right],
\end{aligned}
$$

using [6, Theorem 16.13], it is enough to show that the collection of random variables,

$$
\left\{e^{-r\left(\tau_{\mathscr{O}_{k}} \wedge T-t\right)} u\left(\tau_{\mathscr{O}_{k}} \wedge T, Z\left(\tau_{\mathscr{O}_{k}} \wedge T\right)\right): k \in \mathbb{N}\right\}
$$

is uniformly integrable. For $p_{0}>1$ as in Hypothesis 5.1 it is enough to show that their $p_{0}$-th order moments are uniformly bounded ([6, Observation following Equation (16.23)]); that is,

$$
\sup _{k \in \mathbb{N}} \mathbb{E}_{\mathbb{Q}}^{t, z}\left[\left|e^{-r \tau_{\mathscr{O}_{k}}} u\left(\tau_{\mathscr{O}_{k}}, Z\left(\tau_{\mathscr{O}_{k}}\right)\right) \mathbf{1}_{\left\{\tau_{\mathscr{\sigma}_{k}}<T\right\}}\right|^{p_{0}}\right]<+\infty .
$$

From (1.29), we have, for some constant $C$,

$$
\begin{aligned}
\mathbb{E}_{\mathbb{Q}}^{t, z} & {\left[\left|e^{-r\left(\tau_{\mathscr{C}_{k}} \wedge T-t\right)} u\left(\tau_{\mathscr{O}_{k}} \wedge T, Z\left(\tau_{\mathscr{O}_{k}} \wedge T\right)\right)\right|^{p_{0}}\right] } \\
& \leq C\left(1+\mathbb{E}_{\mathbb{Q}}^{t, z}\left[e^{p_{0} M_{1} Y\left(\tau_{\sigma_{k}} \wedge T\right)}\right]+\mathbb{E}_{\mathbb{Q}}^{t, z}\left[e^{p_{0} M_{2} X\left(\tau_{\mathscr{\sigma}_{k}} \wedge T\right)}\right]\right) .
\end{aligned}
$$

Now, the uniform bound in (5.8) follows by applying the supermartingale property (2.14) with $c:=p_{0} M_{1}$ to the first expectation in the preceding inequality and by 
applying (2.21) with $p:=p_{0} M_{2}$ to the second expectation above. Therefore, by taking the limit as $k$ tends to $\infty$ in (5.4), with the aid of (5.5) and (5.6), we obtain the stochastic representation (1.30) of solutions to problem (5.2).

Proof of Theorem 1.18. The need is to show that if $0<\beta<1$ and $u \in C_{\text {loc }}\left(Q \cup ð^{1} Q\right) \cap$ $C_{s, \text { loc }}^{1,1}\left((0, T) \times\left(\mathscr{O} \cup \Gamma_{0}\right)\right)$ is a solution to problem (1.7) with boundary condition (1.9), satisfying the growth bound (1.29), then it admits the stochastic representation (1.32).

Let $\varepsilon>0$ and $\left\{\mathscr{U}_{k}: k \in \mathbb{N}\right\}$ be the collection of increasing subdomains as in (3.5). By applying Itô's lemma, we obtain

$$
\begin{aligned}
\mathbb{E}_{\mathbb{Q}}^{t, z} & {\left[e^{-r\left(T \wedge \nu \mathscr{U}_{k}\right)} u\left(T \wedge \nu \mathscr{U}_{k}, Z^{\varepsilon}\left(T \wedge \nu \mathscr{U}_{k}\right)\right)\right] } \\
& =u(t, x, y+\varepsilon)-\mathbb{E}_{\mathbb{Q}}^{t, z}\left[\int_{t}^{T \wedge \nu \mathscr{U}_{k}} e^{-r s} A^{\varepsilon} u\left(s, Z^{\varepsilon}(s)\right) d s\right],
\end{aligned}
$$

where $\nu_{\mathscr{U}_{k}}$ is given by (1.22), $Z^{\varepsilon}$ by (3.6) and $A^{\varepsilon}$ is defined by (3.8). The proof now follows the same path as that of Theorem 1.5 .

Analogous to Lemma 2.12, we have the following auxiliary result.

Lemma 5.2. Suppose $f$ and $g$ obey the growth assumption (1.29). Then there are positive constants $\bar{C}, M_{1} \leq \mu$ and $M_{2} \in[0,1]$, such that for any stopping times $\theta_{1}, \theta_{2} \in \mathscr{T}_{t, T}$ with values in $[t, T]$, the function $J_{p}^{\theta_{1}, \theta_{2}}$ given by (1.36) obeys the growth assumption (1.29).

Proof. The proof follows as in Lemma 2.12 with the aid of Lemma 2.11 Notice that because the stopping times $\theta_{1}, \theta_{2} \in \mathscr{T}_{t, T}$ are bounded by $T$, we do not need the constant $r$ to be positive, as in Lemma 2.12 .

Remark 5.3. The function $\psi$ in (1.36) plays the role of the obstacle function and is relevant only for problem (1.8).

Next, we have the following existence results for solutions to the parabolic terminal/boundary value problem (5.2), for all $\beta>0$.

Proof of Theorem 1.22, We choose $T>0$ small enough and $p_{0}>1$ as in Hypothesis 5.1 .

By hypothesis, we have $g \in C_{\text {loc }}\left(\overline{\widetilde{\partial}_{\beta} Q}\right)$. Since $\overline{\widehat{\mathrm{d}}_{\beta} Q}$ is closed, we may use [21, Theorem 3.1.2] to extend $g$ to a function on $[0, T] \times \mathbb{R}^{2}$, again called $g$, such that $g \in C_{\mathrm{loc}}\left([0, T] \times \mathbb{R}^{2}\right)$.

The proof follows the same pattern as that of Theorem 1.8. For completeness, we outline the main steps. Let $\mathscr{O}_{k}$ be an increasing sequence of $C^{2+\alpha}$ subdomains of $\mathscr{O}$ as in the proof of Theorem [1.3, and let $Q_{k}:=(0, T) \times \mathscr{O}_{k}$. We notice that on each cylindrical domain, $Q_{k}$, the operator $A$ is uniformly elliptic, and its coefficients are $C^{\infty}\left(\bar{Q}_{k}\right)$ functions. By hypothesis, there is an $\alpha \in(0,1)$ such that $f \in C^{\alpha}\left(\bar{Q}_{k}\right)$ and $g \in C\left(\bar{Q}_{k}\right)$. Therefore, by [21, Theorem 3.4.9], the terminal value problem

$$
\begin{array}{rlrl}
-u_{t}+A u & =f & & \text { on } Q_{k}, \\
u=g & & \text { on }(0, T) \times \partial \mathscr{O}_{k} \cup\{T\} \times \overline{\mathscr{O}}_{k},
\end{array}
$$


has a unique solution $u_{k} \in C\left(\bar{Q}_{k}\right) \cap C^{2+\alpha}\left(Q_{k}\right)$, and by $7^{7}[24$, Theorem 6.5.2] it has the stochastic representation

$$
\begin{aligned}
u_{k}(t, z)= & \mathbb{E}_{\mathbb{Q}}^{t, z}\left[e^{-r\left(\tau_{\mathscr{O}_{k}} \wedge T-t\right)} g\left(\tau_{\mathscr{O}_{k}} \wedge T, Z\left(\tau_{\mathscr{O}_{k}} \wedge T\right)\right)\right] \\
& +\mathbb{E}_{\mathbb{Q}}^{t, z}\left[\int_{t}^{\tau_{\mathscr{O}_{k}} \wedge T} e^{-r(s-t)} f(s, Z(s)) d s\right], \quad \forall(t, z) \in \bar{Q}_{k} .
\end{aligned}
$$

Because $\tau_{\mathscr{O}_{k}}$ converges to $\tau_{\mathscr{O}}$ a.s. as $k \rightarrow \infty$, the integral term in (5.9) converges to the integral term of $u^{*}$ in (1.30), by the same argument as that used in the proof of Theorem 1.16. By the continuity of $g$ and of the paths of the Heston process $Z$, we also know that

$e^{-r\left(\tau_{\mathscr{O}_{k}} \wedge T\right)} g\left(\tau_{\mathscr{O}_{k}} \wedge T, Z\left(\tau_{\mathscr{O}_{k}} \wedge T\right)\right) \rightarrow e^{-r\left(\tau_{\mathscr{O}} \wedge T\right)} g\left(\tau_{\mathscr{O}} \wedge T, Z\left(\tau_{\mathscr{O}} \wedge T\right)\right), \quad$ as $k \rightarrow \infty$.

In order to show that the preceding convergence takes place in expectation also, it is enough to show that the collection of random variables,

$$
\left\{e^{-r\left(\tau_{\mathscr{O}_{k}} \wedge T\right)} g\left(\tau_{\mathscr{O}_{k}} \wedge T, Z\left(\tau_{\mathscr{O}_{k}} \wedge T\right)\right): k \in \mathbb{N}\right\},
$$

is uniformly integrable, but this follows by the same argument as that used for the collections (5.7) in the proof of Theorem 1.16, by bounding their $p_{0}$-th order moments $\left(p_{0}>1\right)$. Therefore, the sequence $\left\{u_{k}: k \in \mathbb{N}\right\}$ converges to $u^{*}$ pointwise on $Q$. By interior Schauder estimates for parabolic equations [24, Theorem 3.3.5] and Lemma 5.2, there is a subsequence of $\left\{u_{k}: k \in \mathbb{N}\right\}$ which converges to $u^{*}$ in $C^{2+\alpha^{\prime}}(Q)$ when $0<\alpha^{\prime}<\alpha$. Using the Arzelà-Ascoli Theorem, we obtain that $u^{*} \in C^{2+\alpha}(Q)$. The proof of the continuity of $u$ up to $\partial_{\beta} Q$ follows by exactly the same argument as that used in the proof of Step 2 in Theorem 1.8 . Therefore, $u^{*}$ is a solution to (5.2).

From Theorem 1.16 and Lemma 5.2, we see that $u^{*}$ in (1.30) is the unique solution to the parabolic terminal value problem (5.2), for all $\beta>0$.

We now have the

Proof of Theorem 1.23. Just as in the proof of Theorem 1.22, we can easily adapt the proof of Theorem 1.9 for the elliptic case to the present parabolic case. For this purpose, we only need to make use of the local boundary Schauder estimate Lemma A.1] instead of [25, Corollary 6.7] for the elliptic case.

\section{Parabolic obstacle problem}

Problem (1.8) with boundary condition (1.12), when $0<\beta<1$, and with boundary condition (1.9), when $\beta \geq 1$, can be formulated as

$$
\begin{cases}\min \left\{-u_{t}+A u-f, u-\psi\right\}=0 & \text { on } Q, \\ u=g & \text { on } \partial_{\beta} Q,\end{cases}
$$

where $\partial_{\beta} Q$ is defined in (5.1). According to Theorem 1.27, the solution to problem (6.1) is given by (1.37).

\footnotetext{
${ }^{7}$ See also [30, Theorem 5.7.6].
} 
Proof of Theorem 1.27, We choose $\widetilde{T}>0$ small enough so that it obeys Hypothesis 5.1. For such $\widetilde{T}>0$, the proof of Theorem 1.12 adapts to the present case in the same way that the proof of Theorem 1.3 adapts to give a proof of Theorem 1.16. Therefore, it remains to show that the corresponding stochastic representation (1.37) of the solution to problem (6.1) holds for $T$ arbitrarily large.

Let $N:=\lfloor T / \widetilde{T}\rfloor($ the greatest integer in $T / \widetilde{T})$, and $T_{i}:=i \widetilde{T}$, for $i=0, \ldots, N-1$, and $T_{N}:=T$. Let $k$ be an integer such that $1 \leq k \leq N-1$, and assume that the stochastic representation formula (1.37) holds for any $t \in\left[T_{i}, T\right]$, where $i=$ $k, \ldots, N-1$. We want to show that it holds also for $t \in\left[T_{k-1}, T\right]$. Notice that for $k=N-1$, we have $T-t \leq \widetilde{T}$, for all $t \in\left[T_{N-1}, T\right]$, and so we know that the stochastic representation (1.37) of the solution to problem (1.8) holds, by the observation at the beginning of the present proof.

For any $v \in[t, T]$, stopping time $\theta \in \mathscr{T}_{t, v}$ with values in $[t, v]$, and $\varphi \in C(\overline{\mathscr{O}})$, we denote

$$
\begin{aligned}
F^{\varphi}(t, z, v, \theta):= & \int_{t}^{\tau_{\mathscr{O}} \wedge \theta} e^{-r(s-t)} f(s, Z(s)) d s+e^{-r(\theta-t)} \psi(\theta, Z(\theta)) 1_{\left\{\theta<\tau_{\mathscr{O}} \wedge v\right\}} \\
& +e^{-r\left(\tau_{\mathscr{C}}-t\right)} g\left(\tau_{\mathscr{O}}, Z\left(\tau_{\mathscr{O}}\right)\right) 1_{\left\{\tau_{\mathscr{O}} \leq \theta, \tau_{\mathscr{O}}<v\right\}} \\
& +e^{-r(v-t)} \varphi(Z(v)) 1_{\left\{\tau_{\mathscr{O}} \wedge v \leq \theta, \tau_{\mathscr{O}} \geq v\right\}} .
\end{aligned}
$$

Notice that by choosing $\varphi=g(T, \cdot)$ and $v=T$ in (6.2), we obtain, for any stopping time $\theta \in \mathscr{T}_{t, T}$,

$$
\begin{aligned}
& e^{-r\left(\tau_{\mathscr{O}}-t\right)} g\left(\tau_{\mathscr{O}}, Z\left(\tau_{\mathscr{O}}\right)\right) 1_{\left\{\tau_{\mathscr{O}} \leq \theta, \tau_{\mathscr{O}}<T\right\}}+e^{-r(T-t)} \varphi(Z(T)) 1_{\left\{\tau_{\mathscr{C}} \wedge T \leq \theta, \tau_{\mathscr{O}} \geq T\right\}} \\
& =e^{-r\left(\tau_{\mathscr{O}} \wedge T-t\right)} g\left(\tau_{\mathscr{O}} \wedge T, Z\left(\tau_{\mathscr{O}} \wedge T\right)\right) 1_{\left\{\tau_{\mathscr{O}} \wedge T \leq \theta\right\}},
\end{aligned}
$$

and so,

$$
\begin{aligned}
F^{g(T, \cdot)}(t, z, T, \theta)= & \int_{t}^{\tau_{\mathscr{O}} \wedge \theta} e^{-r(s-t)} f(s, Z(s)) d s \\
& +e^{-r(\theta-t)} \psi(\theta, Z(\theta)) 1_{\left\{\theta<\tau_{\mathscr{O}} \wedge T\right\}} \\
& +e^{-r\left(\tau_{\mathscr{O}} \wedge T-t\right)} g\left(\tau_{\mathscr{O}} \wedge T, Z\left(\tau_{\mathscr{O}} \wedge T\right)\right) 1_{\left\{\tau_{\mathscr{O}} \wedge T \leq \theta\right\}}
\end{aligned}
$$

Because $u$ solves problem (6.1) on the interval $\left(T_{k-1}, T_{k}\right)$, and $T_{k}-T_{k-1} \leq \widetilde{T}$, we see that $u$ has the stochastic representation (1.37), for any $t \in\left[T_{k-1}, T_{k}\right)$ and $z \in \mathscr{O} \cup \partial_{\beta} \mathscr{O}:$

$$
u(t, z)=\sup _{\theta \in \mathscr{T}_{t, T_{k}}} \mathbb{E}_{\mathbb{Q}}^{t, z}\left[F^{u^{*}\left(T_{k}, \cdot\right)}\left(t, z, T_{k}, \theta\right)\right]
$$

For any stopping time $\eta \in \mathscr{T}_{t, T_{k}}$, we let

$$
\begin{aligned}
F_{1}\left(t, z, T_{k}, \eta\right):= & \int_{t}^{\tau_{\mathscr{O}} \wedge \eta} e^{-r(s-t)} f(s, Z(s)) d s \\
& +e^{-r(\eta-t)} \psi(\eta, Z(\eta)) \mathbf{1}_{\left\{\eta<\tau_{\mathscr{O}} \wedge T_{k}\right\}} \\
& +e^{-r\left(\tau_{\mathscr{O}}-t\right)} g\left(\tau_{\mathscr{O}}, Z\left(\tau_{\mathscr{O}}\right)\right) \mathbf{1}_{\left\{\tau_{\mathscr{O}} \leq \eta, \eta<T_{k}\right\}}
\end{aligned}
$$


and for any stopping time $\xi \in \mathscr{T}_{T_{k}, T}$, we let

$$
\begin{aligned}
F_{2}(t, z, T, \xi):= & \int_{T_{k}}^{\tau_{\mathscr{O}} \wedge \xi} e^{-r\left(s-T_{k}\right)} f(s, Z(s)) d s \\
& +e^{-r\left(\xi-T_{k}\right)} \psi(\xi, Z(\xi)) \mathbf{1}_{\left\{\xi<\tau_{\mathscr{O}} \wedge T\right\}} \\
& +e^{-r\left(\tau_{\mathscr{O}} \wedge T-T_{k}\right)} g\left(\tau_{\mathscr{O}} \wedge T, Z\left(\tau_{\mathscr{O}} \wedge T\right)\right) \mathbf{1}_{\left\{\tau_{\mathscr{O}} \wedge T \leq \xi\right\}} .
\end{aligned}
$$

For the rest of the proof, we fix $z \in \mathscr{O} \cup \partial_{\beta} \mathscr{O}$ and $t \in\left[T_{k-1}, T_{k}\right)$.

Let $\eta \in \mathscr{T}_{t, T_{k}}$ and $\xi \in \mathscr{T}_{T_{k}, T}$. It is straightforward to see that

$$
\theta:= \begin{cases}\eta & \text { if } \eta<T_{k} \\ \xi & \text { if } \eta=T_{k}\end{cases}
$$

is a stopping time with values in $[t, T]$. We denote

$$
\begin{array}{r}
\mathscr{S}_{t, T}=\left\{\theta \in \mathscr{T}_{t, T}: \theta=\eta \mathbf{1}_{\left\{\eta<T_{k}\right\}}+\xi \mathbf{1}_{\left\{\eta=T_{k}\right\}},\right. \\
\text { where } \left.\eta \in \mathscr{T}_{t, T_{k}} \text { and } \xi \in \mathscr{T}_{T_{k}, T}\right\} .
\end{array}
$$

For any stopping time $\theta \in \mathscr{T}_{t, T}$, we define the stopping times $\theta^{\prime} \in \mathscr{T}_{t, T_{k}}$ and $\theta^{\prime \prime} \in \mathscr{T}_{T_{k}, T}$ by

$$
\theta^{\prime}:=\mathbf{1}_{\left\{\theta<T_{k}\right\}} \theta+\mathbf{1}_{\left\{\theta \geq T_{k}\right\}} T_{k} \quad \text { and } \quad \theta^{\prime \prime}:=\mathbf{1}_{\left\{\theta<T_{k}\right\}} T_{k}+\mathbf{1}_{\left\{\theta \geq T_{k}\right\}} \theta .
$$

Then, any stopping time $\theta \in \mathscr{T}_{t, T}$ can be written as

$$
\begin{aligned}
\theta & =\theta^{\prime} \mathbf{1}_{\left\{\theta<T_{k}\right\}}+\theta^{\prime \prime} \mathbf{1}_{\left\{\theta \geq T_{k}\right\}} \\
& =\theta^{\prime} \mathbf{1}_{\left\{\theta^{\prime}<T_{k}\right\}}+\theta^{\prime \prime} \mathbf{1}_{\left\{\theta^{\prime}=T_{k}\right\}},
\end{aligned}
$$

and so,

$$
\mathscr{T}_{t, T}=\mathscr{S}_{t, T} .
$$

The preceding identity and definitions (1.37) of $u^{*}$ and (6.2) of $F^{\varphi}$ give us

$$
u^{*}(t, z)=\sup _{\theta \in \mathscr{S}_{t, T}} \mathbb{E}_{\mathbb{Q}}^{t, z}\left[F^{g(T, \cdot)}(t, z, T, \theta)\right] .
$$

We shall need the following identities:

Claim 6.1. For any stopping time $\theta=\eta \mathbf{1}_{\left\{\eta<T_{k}\right\}}+\xi \mathbf{1}_{\left\{\eta=T_{k}\right\}}$, where $\eta \in \mathscr{T}_{t, T_{k}}$ and $\xi \in \mathscr{T}_{T_{k}, T}$, we have the following identities:

$$
\begin{aligned}
\int_{t}^{\tau_{\mathscr{O}} \wedge \theta} e^{-r(s-t)} f(s, Z(s)) d s= & \mathbf{1}_{\left\{\eta<T_{k}\right\}} \int_{t}^{\tau_{\mathscr{O}} \wedge \eta} e^{-r(s-t)} f(s, Z(s)) d s \\
& +\mathbf{1}_{\left\{\eta=T_{k}\right\}} \int_{T_{k}}^{\tau_{\mathscr{O}} \wedge \xi} e^{-r(s-t)} f(s, Z(s)) d s,
\end{aligned}
$$

and

$$
\begin{aligned}
e^{-r(\theta-t)} \psi(\theta, Z(\theta)) \mathbf{1}_{\left\{\theta<\tau_{\mathscr{O}} \wedge T\right\}}= & e^{-r(\eta-t)} \psi(\eta, Z(\eta)) \mathbf{1}_{\left\{\eta<\tau_{\mathscr{O}} \wedge T_{k}\right\}} \mathbf{1}_{\left\{\eta<T_{k}\right\}} \\
& +e^{-r(\xi-t)} \psi(\xi, Z(\xi)) \mathbf{1}_{\left\{\xi<\tau_{\mathscr{O}} \wedge T\right\}} \mathbf{1}_{\left\{\eta=T_{k}\right\}},
\end{aligned}
$$

and

$$
\begin{aligned}
e^{-r\left(\tau_{\mathscr{O}} \wedge T-t\right)} g\left(\tau_{\mathscr{O}} \wedge T, Z\left(\tau_{\mathscr{O}} \wedge T\right)\right) \mathbf{1}_{\left\{\tau_{\mathscr{O}} \wedge T \leq \theta\right\}} \\
=e^{-r\left(\tau_{\mathscr{O}}-t\right)} g\left(\tau_{\mathscr{O}}, Z\left(\tau_{\mathscr{O}}\right)\right) \mathbf{1}_{\left\{\tau_{\mathscr{O}} \leq \eta, \eta<T_{k}\right\}} \mathbf{1}_{\left\{\eta<T_{k}\right\}} \\
\quad+e^{-r\left(\tau_{\mathscr{O}} \wedge T-t\right)} g\left(\tau_{\mathscr{O}} \wedge T, Z\left(\tau_{\mathscr{O}} \wedge T\right)\right) \mathbf{1}_{\left\{\tau_{\mathscr{O}} \wedge T \leq \xi\right\}} \mathbf{1}_{\left\{\eta=T_{k}\right\}}
\end{aligned}
$$


Proof. Notice that

$$
\left\{\theta<T_{k}\right\}=\left\{\eta<T_{k}\right\} \text { and }\left\{\theta \geq T_{k}\right\}=\left\{\eta=T_{k}\right\} .
$$

The first identity is obvious because, by (6.10), we see that

$$
\theta=\eta \text { on }\left\{\eta<T_{k}\right\} \text { and } \theta=\xi \text { on }\left\{\eta=T_{k}\right\} .
$$

The second identity follows by the observation that

$$
\left\{\theta<\tau_{\mathscr{O}} \wedge T\right\}=\left\{\theta<\tau_{\mathscr{O}} \wedge T, \theta<T_{k}\right\} \cup\left\{\theta<\tau_{\mathscr{O}} \wedge T, \theta \geq T_{k}\right\},
$$

and using (6.11) and (6.10), it follows that

$$
\left\{\theta<\tau_{\mathscr{O}} \wedge T\right\}=\left\{\eta<\tau_{\mathscr{O}} \wedge T_{k}, \eta<T_{k}\right\} \cup\left\{\xi<\tau_{\mathscr{O}} \wedge T, \eta=T_{k}\right\} .
$$

For the last identity of the claim, we notice that

$$
\begin{aligned}
\left\{\tau_{\mathscr{O}} \wedge T \leq \theta\right\}= & \left\{\tau_{\mathscr{O}} \wedge T \leq \theta, \tau_{\mathscr{O}}<T\right\} \cup\left\{\tau_{\mathscr{O}} \wedge T \leq \theta, \tau_{\mathscr{O}} \geq T\right\} \\
= & \left\{\tau_{\mathscr{O}} \wedge T \leq \theta, \tau_{\mathscr{O}}<T, \theta<T_{k}\right\} \cup\left\{\tau_{\mathscr{O}} \wedge T \leq \theta, \tau_{\mathscr{O}}<T, \theta \geq T_{k}\right\} \\
& \cup\left\{\tau_{\mathscr{O}} \wedge T \leq \theta, \tau_{\mathscr{O}} \geq T\right\} .
\end{aligned}
$$

By (6.11) and (6.10), we obtain

$$
\begin{aligned}
\left\{\tau_{\mathscr{O}} \wedge T \leq \theta\right\}= & \left\{\tau_{\mathscr{O}} \leq \eta, \tau_{\mathscr{O}}<T, \eta<T_{k}\right\} \cup\left\{\tau_{\mathscr{O}} \wedge T \leq \xi, \tau_{\mathscr{O}}<T, \eta=T_{k}\right\} \\
& \cup\left\{\tau_{\mathscr{O}} \wedge T \leq \xi, \tau_{\mathscr{O}} \geq T\right\} \\
= & \left\{\tau_{\mathscr{O}} \leq \eta, \eta<T_{k}\right\} \cup\left\{\tau_{\mathscr{O}} \wedge T \leq \xi, \eta=T_{k}\right\}
\end{aligned}
$$

which implies the last identity of the claim.

We can write the expression for $F^{g(T, \cdot)}(t, z, T, \theta)$ as a sum:

$$
F^{g(T, \cdot)}(t, z, T, \theta)=\mathbf{1}_{\left\{\eta<T_{k}\right\}} F_{1}\left(t, z, T_{k}, \eta\right)+\mathbf{1}_{\left\{\eta=T_{k}\right\}} e^{-r\left(T_{k}-t\right)} F_{2}(t, z, T, \xi) .
$$

Because $\xi \in \mathscr{T}_{T_{k}, T}$ and $F_{2}(t, z, T, \xi)$ depends only on $\left(Z^{t, z}(s)\right)_{T_{k}<s<T}$, and the Heston process has the (strong) Markov property [16. Theorem $1.1 \overline{5}(\mathrm{c})$ ], we have a.s. that

$$
\begin{aligned}
\mathbb{E}_{\mathbb{Q}}^{t, z}\left[F_{2}(t, z, T, \xi) \mid \mathscr{F}_{T_{k}}\right] & =\mathbb{E}_{\mathbb{Q}}^{T_{k}, Z^{t, z}\left(T_{k}\right)}\left[F_{2}\left(T_{k}, Z^{t, z}\left(T_{k}\right), T, \xi\right)\right] \\
& =\mathbb{E}_{\mathbb{Q}}^{T_{k}, Z^{t, z}\left(T_{k}\right)}\left[F^{g(T, \cdot)}\left(T_{k}, Z^{t, z}\left(T_{k}\right), T, \xi\right)\right],
\end{aligned}
$$

by applying definitions (6.3) and (6.6). Thus,

$$
\begin{aligned}
\mathbb{E}_{\mathbb{Q}}^{t, z} & {\left[\mathbf{1}_{\left\{\eta=T_{k}\right\}} e^{-r\left(T_{k}-t\right)} F_{2}(t, z, T, \xi)\right] } \\
& =\mathbb{E}_{\mathbb{Q}}^{t, z}\left[\mathbb{E}_{\mathbb{Q}}^{t, z}\left[\mathbf{1}_{\left\{\eta=T_{k}\right\}} e^{-r\left(T_{k}-t\right)} F_{2}(t, z, T, \xi) \mid \mathscr{F}_{T_{k}}\right]\right] \\
& =\mathbb{E}_{\mathbb{Q}}^{t, z}\left[\mathbf{1}_{\left\{\eta=T_{k}\right\}} e^{-r\left(T_{k}-t\right)} \mathbb{E}_{\mathbb{Q}}^{t, z}\left[F_{2}(t, z, T, \xi) \mid \mathscr{F}_{T_{k}}\right]\right] \\
& =\mathbb{E}_{\mathbb{Q}}^{t, z}\left[\mathbf{1}_{\left\{\eta=T_{k}\right\}} e^{-r\left(T_{k}-t\right)} \mathbb{E}_{\mathbb{Q}}^{T_{k}, Z\left(T_{k}\right)}\left[F^{g(T, \cdot)}\left(T_{k}, Z\left(T_{k}\right), T, \xi\right)\right]\right]
\end{aligned}
$$


By the preceding identity, (6.7) and (6.12), the identity (6.9) yields

$$
\begin{aligned}
u^{*}(t, z)= & \sup _{\substack{\theta=\eta \mathbf{1}_{\left\{\eta<T_{k}\right\}}+\xi \mathbf{1}_{\left\{\eta=T_{k}\right\}} \\
\theta \in \mathscr{S}_{t, T}, \eta \in \mathscr{T}_{t, T_{k}}, \xi \in \mathscr{T}_{T_{k}, T}}}\left\{\mathbb { E } _ { \mathbb { Q } } ^ { t , z } \left[\mathbf{1}_{\left\{\eta<T_{k}\right\}} F_{1}\left(t, z, T_{k}, \eta\right)\right.\right. \\
+ & \left.\left.\mathbf{1}_{\left\{\eta=T_{k}\right\}} e^{-r\left(T_{k}-t\right)} \mathbb{E}_{\mathbb{Q}}^{T_{k}, Z\left(T_{k}\right)}\left[F^{g(T, \cdot)}\left(T_{k}, Z\left(T_{k}\right), T, \xi\right)\right]\right]\right\} \\
= & \sup _{\eta \in \mathscr{T}_{t, T_{k}}}\left\{\mathbb { E } _ { \mathbb { Q } } ^ { t , z } \left[\mathbf{1}_{\left\{\eta<T_{k}\right\}} F_{1}\left(t, z, T_{k}, \eta\right)\right.\right. \\
+ & \left.\left.\mathbf{1}_{\left\{\eta=T_{k}\right\}} e^{-r\left(T_{k}-t\right)} \sup _{\xi \in \mathscr{T}_{T_{k}, T}} \mathbb{E}_{\mathbb{Q}}^{T_{k}, Z\left(T_{k}\right)}\left[F^{g(T, \cdot)}\left(T_{k}, Z\left(T_{k}\right), T, \xi\right)\right]\right]\right\} .
\end{aligned}
$$

Using the definition (1.37) of $u^{*}$, we have

$$
u^{*}\left(T_{k}, Z\left(T_{k}\right)\right)=\sup _{\xi \in \mathscr{T}_{T_{k}, T}} \mathbb{E}_{\mathbb{Q}}^{T_{k}, Z\left(T_{k}\right)}\left[F^{g(T, \cdot)}\left(T_{k}, Z\left(T_{k}\right), T, \xi\right)\right]
$$

and so it follows that

$$
u^{*}(t, z)=\sup _{\eta \in \mathscr{T}_{t, T_{k}}} \mathbb{E}_{\mathbb{Q}}^{t, z}\left[\mathbf{1}_{\left\{\eta<T_{k}\right\}} F_{1}\left(t, z, T_{k}, \eta\right)+\mathbf{1}_{\left\{\eta=T_{k}\right\}} e^{-r\left(T_{k}-t\right)} u^{*}\left(T_{k}, Z\left(T_{k}\right)\right)\right] .
$$

Notice that, by definitions (6.2) of $F^{\varphi}$ and (6.5) of $F_{1}$, we have

$$
F^{u^{*}(T, \cdot)}\left(t, z, T_{k}, \eta\right)=\mathbf{1}_{\left\{\eta<T_{k}\right\}} F_{1}\left(t, z, T_{k}, \eta\right)+\mathbf{1}_{\left\{\eta=T_{k}\right\}} e^{-r\left(T_{k}-t\right)} u^{*}\left(T_{k}, Z\left(T_{k}\right)\right) .
$$

The preceding two identities yield

$$
\begin{aligned}
u^{*}(t, z) & =\sup _{\eta \in \mathscr{T}_{t, T_{k}}} \mathbb{E}_{\mathbb{Q}}^{t, z}\left[F^{u^{*}(T, \cdot)}\left(t, z, T_{k}, \eta\right)\right] \\
& =u(t, z) \quad(\text { by }(\underline{6.4})) .
\end{aligned}
$$

This concludes the proof of the theorem.

Proof of Theorem 1.28, We omit the proof, as it is very similar to the proofs of Theorems 1.27 and 1.13.

\section{APPENDIX A. LOCAL A PRIORI BOUNDARY ESTIMATES}

To complete the proof of Theorem 1.23 we need the following local a priori boundary estimate (a parabolic analogue of [25, Corollary 6.7]) for a solution to a parabolic terminal/boundary value problem and for which we were not able to find a suitable reference in the literature.

Lemma A.1 (Local a priori boundary estimates). Let $\mathscr{O} \subseteq \mathbb{H}$ be a domain such that the boundary portion $\Gamma_{1}$ is of class $C^{2+\alpha}$. For $z_{0} \in \Gamma_{1}$ and $R>0$, let

$$
B_{R}\left(z_{0}\right):=\left\{z \in \mathbb{R}^{d}:\left|z-z_{0}\right|<R\right\} \quad \text { and } \quad Q_{R, T}\left(z_{0}\right):=(0, T) \times \mathscr{O} \cap B_{R}\left(z_{0}\right) .
$$

Assume $B_{2 R}\left(z_{0}\right) \Subset \mathbb{H}$ and let $f \in C^{\alpha}\left(\bar{Q}_{2 R, T}\left(z_{0}\right)\right)$ and $g \in C^{2+\alpha}\left(\bar{Q}_{2 R, T}\left(z_{0}\right)\right)$. Then, there is a positive constant $C$, depending only on $z_{0}, R$ and the coefficients of $A$, 
such that for any solution $u \in C^{2+\alpha}\left(\bar{Q}_{2 R, T}\left(z_{0}\right)\right)$ to

$$
\begin{aligned}
-u_{t}+A u=f & \text { on } Q_{2 R, T}\left(z_{0}\right), \\
u=g & \text { on }\left((0, T] \times\left(B_{2 R}\left(z_{0}\right) \cap \Gamma_{1}\right)\right) \cup\left(\{T\} \times \mathscr{O} \cap B_{2 R}\left(z_{0}\right)\right),
\end{aligned}
$$

we have

$$
\|u\|_{C^{2+\alpha}\left(\bar{Q}_{R, T}\left(z_{0}\right)\right)} \leq C\left(\|f\|_{C^{\alpha}\left(\bar{Q}_{2 R, T}\left(z_{0}\right)\right)}+\|g\|_{C^{2+\alpha}\left(\bar{Q}_{2 R, T}\left(z_{0}\right)\right)}+\|u\|_{C\left(\bar{Q}_{2 R, T}\left(z_{0}\right)\right)}\right) .
$$

Proof. The result follows by combining the global Schauder estimate 34, Theorem 10.4.1] and the localization procedure of [34, Theorem 8.11.1], exactly as in the proof of [16, Theorem 3.8].

Remark A.2. The interior version of Lemma A.1 can be found in [34, Exercise 10.4.2].

\section{Appendix B. Regular points and CONTINUity PROPERTIES OF STOCHASTIC REPRESENTATIONS}

For the purpose of this section, we let $d$ be a non-negative integer, $D \subset \mathbb{R}^{d}$ a bounded domain and $t_{1}<t_{2}$. We denote $Q:=\left(t_{1}, t_{2}\right) \times D$ and recall that $ð Q:=\left(t_{1}, t_{2}\right) \times \partial D \cup\left\{t_{2}\right\} \times \bar{D}$. We consider coefficients $a, b$ and $\sigma$ satisfying the following conditions.

\section{Hypothesis B.1. Let}

$$
a: \bar{Q} \rightarrow \mathbb{R}^{d \times d} \quad \text { and } \quad b: \bar{Q} \rightarrow \mathbb{R}^{d}
$$

be maps with component functions, $a^{i j}, b^{i}$, belonging to $C^{0,1}(\bar{Q})$. Require that the matrix, a, be symmetric and obey

$$
\sum_{i, j=1}^{d} a^{i j}(t, z) \xi^{i} \xi^{j} \geq \delta|\xi|^{2}, \quad \forall \xi \in \mathbb{R}^{d}, \quad \forall(t, z) \in \bar{Q},
$$

where $\delta$ is a positive constant.

Let $\sigma$ be a square root of the matrix $a$ such that $\sigma \in C^{0,1}\left(\bar{Q} ; \mathbb{R}^{d \times d}\right)$. Such a choice exists by [24, Lemma 6.1.1]. We consider extensions of the coefficients $b$ and $\sigma$ from $\bar{Q}$ to $\mathbb{R} \times \mathbb{R}^{d}$, such that these extensions are bounded and uniformly Lipschitz continuous, and condition (B.1) is satisfied on $\mathbb{R} \times \mathbb{R}^{d}$. Then, by [30, Theorems 5.2.5 \& 5.2.9], for any $(t, z) \in \mathbb{R} \times \mathbb{R}^{d}$ there is a unique strong solution to

$$
\begin{aligned}
d Z_{i}(s) & =b^{i}(s, Z(s)) d t+\sum_{j=1}^{d} \sigma^{i j}(s, Z(s)) d W_{j}(s), \quad \forall i=1, \ldots, d, \quad s>t, \\
Z(t) & =z
\end{aligned}
$$

where $W$ is a $\mathbb{R}^{d}$-valued Brownian motion. Let $\tau_{Q}$ be the first exit time of the process $Z$ from $Q$.

We have the following consequence of 8 [12, Theorem 2.4.2] and [12, Theorem 2.4.1 and the Remark following Theorem 2.4.1].

\footnotetext{
${ }^{8}$ See also [30, Theorem 4.2.12].
} 
Corollary B.2 (Continuity of stochastic representations with killing term). Let $-\infty<t_{1}<t_{2} \leq+\infty$ and $D \subseteq \mathbb{R}^{d}$ be a domain. Let $Q=\left(t_{1}, t_{2}\right) \times D$. Assume Hypothesis B.1 holds and

(1) the function $g$ is Borel measurable, bounded on $\partial Q$ and continuous at all regular points, $(t, z) \in \partial Q$,

(2) if $t_{2}=\infty$, the function $c: \bar{Q} \rightarrow[0, \infty)$ is non-negative, bounded and Borel measurable,

(3) if $t_{2}<\infty$, the function $c: \bar{Q} \rightarrow \mathbb{R}$ is bounded and Borel measurable.

Then

$$
\lim _{Q \ni\left(t^{\prime}, z^{\prime}\right) \rightarrow(t, z)} \mathbb{E}_{\mathbb{Q}}^{t^{\prime}, z^{\prime}}\left[\exp \left(-\int_{t^{\prime}}^{\tau_{Q}} c(s, Z(s)) d s\right) g\left(\tau_{Q}, Z\left(\tau_{Q}\right)\right)\right]=g(t, z),
$$

for all regular points $(t, z) \in \partial Q$.

Proof. We first consider the case when the stopping time $\tau_{Q}$ is not necessarily bounded by a positive constant $T$. Then, we let $c_{0}$ be a positive constant such that

$$
0 \leq c \leq c_{0}, \quad \text { a.e. on } Q
$$

Let $(t, z) \in \partial Q$ be a fixed regular point. We fix $\varepsilon>0$ and consider $t^{\prime} \in\left[t_{1}, t_{2}\right]$ such that $\left|t-t^{\prime}\right|<\varepsilon / 2$. Then, using the fact that $\tau_{Q}^{t^{\prime}, z^{\prime}} \geq t^{\prime}>t-\varepsilon / 2$, we see that

$$
\left\{\tau_{Q}^{t^{\prime}, z^{\prime}}<t-\varepsilon\right\} \subseteq\left\{t-\varepsilon / 2<t^{\prime} \leq \tau_{Q}^{t^{\prime}, z^{\prime}}<t-\varepsilon\right\}=\varnothing
$$

and so, we obtain

$$
\begin{aligned}
\left\{\left|\tau_{Q}^{t^{\prime}, z^{\prime}}-t\right|>\varepsilon\right\} & \subseteq\left\{\tau_{Q}^{t^{\prime}, z^{\prime}}>t+\varepsilon\right\} \cup\left\{\tau_{Q}^{t^{\prime}, z^{\prime}}<t-\varepsilon\right\} \\
& =\left\{\tau_{Q}^{t^{\prime}, z^{\prime}}>t+\varepsilon\right\}
\end{aligned}
$$

Applying [12, Theorem 2.4.1 and the Remark following Theorem 2.4.1] with $t_{0}:=$ $t+\varepsilon$, it follows that

$$
\lim _{Q \ni\left(t^{\prime}, z^{\prime}\right) \rightarrow(t, z)} \mathbb{Q}^{t^{\prime}, z^{\prime}}\left(\left|\tau_{Q}-t\right|>\varepsilon\right) \leq \lim _{Q \ni\left(t^{\prime}, z^{\prime}\right) \rightarrow(t, z)} \mathbb{Q}^{t^{\prime}, z^{\prime}}\left(\tau_{Q}>t+\varepsilon\right)=0
$$

from where it follows that $\tau_{Q}^{t^{\prime}, z^{\prime}}$ converges in probability to 0. Similarly, we can argue that

$$
\exp \left(-\int_{t^{\prime}}^{\tau_{Q}^{t^{\prime}, z^{\prime}}} c\left(s, Z^{\left(t^{\prime}, z^{\prime}\right)}(s)\right) d s\right)
$$

converges in probability to 1 , as $\left(t^{\prime}, z^{\prime}\right) \in Q$ tends to $(t, z)$. We again fix $\varepsilon \in(0,1)$ and consider $t^{\prime}$ such that $\left|t^{\prime}-t\right|<-\left(1 /\left(2 c_{0}\right)\right) \log (1-\varepsilon)$. By inequality (B.4), we 
see that

$$
\begin{aligned}
\mathbb{Q}^{t^{\prime}, z^{\prime}} & \left(\left|\exp \left(-\int_{t^{\prime}}^{\tau_{Q}} c(s, Z(s)) d s\right)-1\right|>\varepsilon\right) \\
& =\mathbb{Q}^{t^{\prime}, z^{\prime}}\left(\exp \left(-\int_{t^{\prime}}^{\tau_{Q}} c(s, Z(s)) d s\right)<1-\varepsilon\right) \quad(\text { as } c \geq 0) \\
& \leq \mathbb{Q}^{t^{\prime}, z^{\prime}}\left(\exp \left(-c_{0}\left(\tau_{Q}-t^{\prime}\right)\right)<1-\varepsilon\right) \quad\left(\text { as } 0 \leq c \leq c_{0}\right) \\
& =\mathbb{Q}^{t^{\prime}, z^{\prime}}\left(\tau_{Q}>t^{\prime}-\frac{1}{c_{0}} \log (1-\varepsilon)\right) \\
& \left.=\mathbb{Q}^{t^{\prime}, z^{\prime}}\left(\tau_{Q}>t-\frac{1}{2 c_{0}} \log (1-\varepsilon)\right) \quad \text { (because }\left|t^{\prime}-t\right|<-\left(1 /\left(2 c_{0}\right)\right) \log (1-\varepsilon)\right) .
\end{aligned}
$$

Choosing $t_{0}:=t-(\log (1-\varepsilon)) /\left(2 c_{0}\right)$ in [12, Theorem 2.4.1 and the Remark following Theorem 2.4.1], we see that the last term in the preceding sequence of inequalities converges to 0 , and so the collection of random variables in (B.5) converges in probability to 1 , as $\left(t^{\prime}, z^{\prime}\right) \in Q$ tends to $(t, z)$. The sequence is uniformly bounded by the constant 1, and so [20, Exercise 2.4.34 (b)] implies that the sequence converges to 1 in expectation also; that is,

$$
\lim _{Q \ni\left(t^{\prime}, z^{\prime}\right) \rightarrow(t, z)} \mathbb{E}_{\mathbb{Q}}^{t^{\prime}, z^{\prime}}\left[\left|\exp \left(-\int_{t^{\prime}}^{\tau_{Q}} c(s, Z(s)) d s\right)-1\right|\right]=0 .
$$

From the sequence of inequalities,

$$
\begin{aligned}
\left|\mathbb{E}_{\mathbb{Q}}^{t^{\prime}, z^{\prime}}\left[\exp \left(-\int_{t^{\prime}}^{\tau_{Q}} c(s, Z(s)) d s\right) g\left(\tau_{Q}, Z\left(\tau_{Q}\right)\right)\right]-g(t, z)\right| \\
\leq\left|\mathbb{E}_{\mathbb{Q}}^{t^{\prime}, z^{\prime}}\left[g\left(\tau_{Q}, Z\left(\tau_{Q}\right)\right)\right]-g(t, z)\right| \\
\quad+\left|\mathbb{E}_{\mathbb{Q}}^{t^{\prime}, z^{\prime}}\left[\left(1-\exp \left(-\int_{t^{\prime}}^{\tau_{Q}} c(s, Z(s)) d s\right)\right) g\left(\tau_{Q}, Z\left(\tau_{Q}\right)\right)\right]\right| \\
\leq\left|\mathbb{E}_{\mathbb{Q}}^{t^{\prime}, z^{\prime}}\left[g\left(\tau_{Q}, Z\left(\tau_{Q}\right)\right)\right]-g(t, z)\right| \\
\quad+\|g\|_{L^{\infty}(ð Q)} \mathbb{E}_{\mathbb{Q}}^{t^{\prime}, z^{\prime}}\left[\left|1-\exp \left(-\int_{t^{\prime}}^{\tau_{Q}} c(s, Z(s)) d s\right)\right|\right],
\end{aligned}
$$

the conclusion (B.3) follows from (B.6) and [12, Theorem 2.4.2], which shows that

$$
\lim _{Q \ni\left(t^{\prime}, z^{\prime}\right) \rightarrow(t, z)} \mathbb{E}_{\mathbb{Q}}^{t^{\prime}, z^{\prime}}\left[g\left(\tau_{Q}, Z\left(\tau_{Q}\right)\right)\right]=g(t, z) .
$$

We next consider the case when $t_{2}<\infty$, and so, by choosing $T=t_{2}$, the stopping time $\tau_{Q}$ is bounded a.s. by $T$. We fix $(t, z) \in \partial Q$. Without loss of generality, we may assume that $t \in[0, T]$ and $Q \subseteq[0, T] \times \mathbb{R}^{d}$. Because $c$ is a bounded function on $Q$, we let $c_{1}, c_{2}$ be two positive constants such that

$$
-c_{1} \leq c \leq c_{2} \text { a.e. on } Q
$$

and we set

$$
\tilde{c}:=c+c_{1} \quad \text { on } Q
$$

and

$$
\tilde{g}\left(t^{\prime}, z^{\prime}\right):=e^{c_{1}\left(t^{\prime}-t\right)} g\left(t^{\prime}, z^{\prime}\right), \quad \forall\left(t^{\prime}, z^{\prime}\right) \in \check{Q}
$$


Notice that $\tilde{c}$ is a non-negative, bounded Borel measurable function on $Q$. Also, $\tilde{g}$ is a bounded, Borel measurable function on $\precsim Q$, and it is continuous at $(t, z)$ with

$$
\tilde{g}(t, z)=g(t, z) \text {. }
$$

In addition, we have for all $\left(t^{\prime}, z^{\prime}\right) \in Q$,

$$
\begin{aligned}
& \exp \left(-\int_{t^{\prime}}^{\tau_{Q}} c\left(s, Z^{t^{\prime}, z^{\prime}}(s)\right) d s\right) g\left(\tau_{Q}, Z^{t^{\prime}, z^{\prime}}\left(\tau_{Q}\right)\right) \\
& =\exp \left(-\int_{t^{\prime}}^{\tau_{Q}} \tilde{c}\left(s, Z^{t^{\prime}, z^{\prime}}(s)\right) d s\right) \tilde{g}\left(\tau_{Q}, Z^{t^{\prime}, z^{\prime}}\left(\tau_{Q}\right)\right) \\
& \quad+\left(\exp \left(c_{1}\left(t-t^{\prime}\right)\right)-1\right) \exp \left(-\int_{t^{\prime}}^{\tau_{Q}} \tilde{c}\left(s, Z^{t^{\prime}, z^{\prime}}(s)\right) d s\right) \tilde{g}\left(\tau_{Q}, Z^{t^{\prime}, z^{\prime}}\left(\tau_{Q}\right)\right) .
\end{aligned}
$$

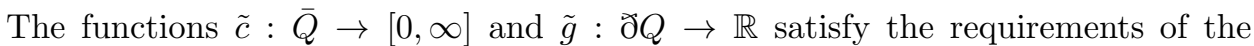
preceding case, and so we have that

$$
\lim _{Q \ni\left(t^{\prime}, z^{\prime}\right) \rightarrow(t, z)} \mathbb{E}_{\mathbb{Q}}^{t^{\prime}, z^{\prime}}\left[\exp \left(-\int_{t^{\prime}}^{\tau_{Q}} \tilde{c}(s, Z(s)) d s\right) \tilde{g}\left(\tau_{Q}, Z\left(\tau_{Q}\right)\right)\right]=g(t, z),
$$

using (B.7). By the boundedness of $\tilde{c}$ on $Q$, of $\tilde{g}$ on $\partial Q$, and the fact that $\tau_{Q} \leq T$ a.s., we also have

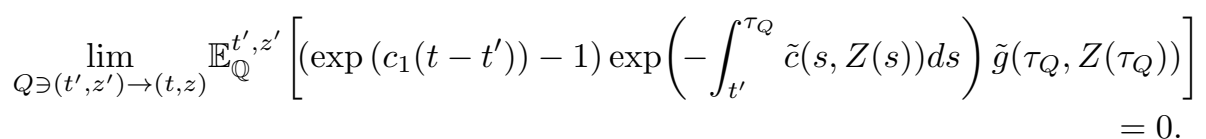

Therefore, the conclusion of the corollary follows from the preceding two limits and identity (B.8).

\section{ACKNOWLEDGMENTS}

We are very grateful to everyone who has provided us with comments on previous versions of this article or related conference or seminar presentations. The second author thanks Daniel Ocone for many helpful discussions on probability theory. Finally, we thank the anonymous referee for many helpful suggestions and kind comments.

\section{REFERENCES}

[1] M. Abramovitz and I. A. Stegun, Handbook of mathematical functions, Dover, New York, 1972.

[2] R. A. Adams, Sobolev spaces, Academic Press [A subsidiary of Harcourt Brace Jovanovich, Publishers], New York-London, 1975. Pure and Applied Mathematics, Vol. 65. MR0450957 (56 \#9247)

[3] L. Andersen, Simple and efficient simulation of the Heston stochastic volatility model, Journal of Computational Finance 11 (2008), 1-42.

[4] E. Bayraktar, C. Kardaras, and H. Xing, Valuation equations for stochastic volatility models, SIAM J. Financial Math. 3 (2012), 351-373. MR.2968038

[5] A. Bensoussan and J.-L. Lions, Applications of variational inequalities in stochastic control, Studies in Mathematics and its Applications, vol. 12, North-Holland Publishing Co., Amsterdam, 1982. Translated from the French. MR653144 (83e:49012)

[6] P. Billingsley, Probability and measure, 2nd ed., Wiley Series in Probability and Mathematical Statistics: Probability and Mathematical Statistics, John Wiley \& Sons Inc., New York, 1986. MR.830424(87f:60001) 
[7] P. Constantin and G. Iyer, A stochastic Lagrangian representation of the three-dimensional incompressible Navier-Stokes equations, Comm. Pure Appl. Math. 61 (2008), 330-345, DOI 10.1002/cpa.20192. MR.2376844(2009b:35319)

[8] P. Daskalopoulos and P. M. N. Feehan, Existence, uniqueness, and global regularity for variational inequalities and obstacle problems for degenerate elliptic partial differential operators in mathematical finance, arxiv.org/abs/1109.1075v1.

[9] P. Daskalopoulos and P. M. N. Feehan, $C^{1,1}$ regularity for degenerate elliptic obstacle problems in mathematical finance, arXiv:1206.0831.

[10] P. Daskalopoulos and P. M. N. Feehan, Existence, uniqueness, and global regularity for variational inequalities and obstacle problems for degenerate parabolic partial differential operators in mathematical finance, in preparation.

[11] P. Daskalopoulos and R. Hamilton, Regularity of the free boundary for the porous medium equation, J. Amer. Math. Soc. 11 (1998), 899-965, DOI 10.1090/S0894-0347-98-00277-X. MR.1623198 (99d:35182)

[12] E. B. Dynkin, Diffusions, superdiffusions and partial differential equations, American Mathematical Society Colloquium Publications, vol. 50, American Mathematical Society, Providence, RI, 2002. MR1883198 (2003c:60001)

[13] E. Ekström and J. Tysk, The Black-Scholes equation in stochastic volatility models, J. Math. Anal. Appl. 368 (2010), 498-507, DOI 10.1016/j.jmaa.2010.04.014. MR2643818 (2011d:60172)

[14] E. Ekström and J. Tysk, Boundary conditions for the single-factor term structure equation, Ann. Appl. Probab. 21 (2011), 332-350, DOI 10.1214/10-AAP698. MR2759205 (2012d:91247)

[15] P. M. N. Feehan and C. A. Pop, Degenerate elliptic operators in mathematical finance and Hölder continuity for solutions to variational equations and inequalities, arxiv.org/abs/ $1110.5594 \mathrm{v} 3$.

[16] P. M. N. Feehan and C. A. Pop, On the martingale problem for degenerate-parabolic partial differential operators with unbounded coefficients and a mimicking theorem for Itô processes, Trans. Amer. Math. Soc., to appear, arXiv:1211.4636.

[17] P. M. N. Feehan and C. A. Pop, Schauder a priori estimates and regularity of solutions to degenerate-elliptic linear second-order partial differential equations, J. Differential Equations 256 (2014), 895-956. MR.3128929

[18] P. M. N. Feehan and C. A. Pop, A Schauder approach to degenerate-parabolic partial differential equations with unbounded coefficients, J. Differential Equations 254 (2013), 4401-4445. MR3040945

[19] R. P. Feynman, The principle of least action in quantum mechanics, Ph.D. thesis, Princeton University, Princeton, NJ, 1942, reprinted in "Feynman's Thesis: a New Approach to Quantum Theory", L. M. Brown (ed.), World Scientific, New York, 2005.

[20] G. B. Folland, Real analysis, 2nd ed., Pure and Applied Mathematics (New York), John Wiley \& Sons Inc., New York, 1999. Modern techniques and their applications; A Wiley-Interscience Publication. MR 1681462 (2000c:00001)

[21] A. Friedman, Partial differential equations of parabolic type, Prentice-Hall Inc., Englewood Cliffs, N.J., 1964. MR0181836 (31 \#6062)

[22] A. Friedman, Uniqueness for the Cauchy problem for degenerate parabolic equations, Pacific J. Math. 46 (1973), 131-147. MR0330773 (48 \#9110)

[23] A. Friedman, Fundamental solutions for degenerate parabolic equations, Acta Math. 133 (1974), 171-217. MR0481551 (58 \#1665)

[24] A. Friedman, Stochastic differential equations and applications, vol. I, II, Academic, New York, 1975 and 1976. MR0494490, MR0494491

[25] D. Gilbarg and N. S. Trudinger, Elliptic partial differential equations of second order, 2nd ed., Grundlehren der Mathematischen Wissenschaften [Fundamental Principles of Mathematical Sciences], vol. 224, Springer-Verlag, Berlin, 1983. MR737190 (86c:35035)

[26] P. Glasserman, Monte Carlo methods in financial engineering, Springer, New York, 2004. MR.1999614 (20049:65005)

[27] S. Heston, A closed-form solution for options with stochastic volatility with applications to bond and currency options, Review of Financial Studies 6 (1993), 327-343. 
[28] N. Ikeda and S. Watanabe, Stochastic differential equations and diffusion processes, NorthHolland Mathematical Library, vol. 24, North-Holland Publishing Co., Amsterdam, 1981. MR 637061 (84b:60080)

[29] M. Kac, On distributions of certain Wiener functionals, Trans. Amer. Math. Soc. 65 (1949), 1-13. MR0027960 (10,383b)

[30] I. Karatzas and S. E. Shreve, Brownian motion and stochastic calculus, 2nd ed., Graduate Texts in Mathematics, vol. 113, Springer-Verlag, New York, 1991. MR1121940 (92h:60127)

[31] I. Karatzas and S. E. Shreve, Methods of mathematical finance, Applications of Mathematics (New York), vol. 39, Springer-Verlag, New York, 1998. MR1640352 (2000e:91076)

[32] S. Karlin and Taylor, A second course on stochastic processes, Academic, New York, 1981. MR 0611513 (82J:60003)

[33] H. Koch, Non-Euclidean singular integrals and the porous medium equation, Habilitation Thesis, University of Heidelberg, 1999, www.mathematik.uni-dortmund.de/lsi/koch/ publications.html.

[34] N. V. Krylov, Lectures on elliptic and parabolic equations in Hölder spaces, Graduate Studies in Mathematics, vol. 12, American Mathematical Society, Providence, RI, 1996. MR 1406091 (97i:35001)

[35] G. M. Lieberman, Second order parabolic differential equations, World Scientific Publishing Co. Inc., River Edge, NJ, 1996. MR1465184 (98k:35003)

[36] R. Lord, R. Koekkoek, and D. Van Dijk, A comparison of biased simulation schemes for stochastic volatility models, Quant. Finance 10 (2010), 177-194, DOI 10.1080/14697680802392496. MR2642962 (2011e:91164)

[37] J. Lörinczi, F. Hiroshima, and V. Betz, Feynman-Kac-type theorems and Gibbs measures on path space, de Gruyter, Berlin, 2011. MR2848339 (2012h:58014)

[38] B. Øksendal, Stochastic differential equations, 6th ed., Universitext, Springer-Verlag, Berlin, 2003. An introduction with applications. MR2001996 (2004e:60102)

[39] O. A. Olĕnik and E. V. Radkevič, Second order equations with nonnegative characteristic form, Plenum Press, New York, 1973. MR0457908

[40] D. Revuz and M. Yor, Continuous martingales and Brownian motion, 3rd ed., Grundlehren der Mathematischen Wissenschaften [Fundamental Principles of Mathematical Sciences], vol. 293, Springer-Verlag, Berlin, 1999. MR1725357 (2000h:60050)

[41] B. Simon, Functional integration and quantum physics, 2nd ed., AMS Chelsea Publishing, Providence, RI, 2005. MR2105995 (2005f:81003)

[42] A. V. Skorokhod, Studies in the theory of random processes, Translated from the Russian by Scripta Technica, Inc, Addison-Wesley Publishing Co., Inc., Reading, Mass., 1965. MR0185620 (32 \#3082b)

[43] D. Stroock and S. R. S. Varadhan, On degenerate elliptic-parabolic operators of second order and their associated diffusions, Comm. Pure Appl. Math. 25 (1972), 651-713. MR0387812 (52 \#8651)

[44] T. Yamada and S. Watanabe, On the uniqueness of solutions of stochastic differential equations, J. Math. Kyoto Univ. 11 (1971), 155-167. MR0278420(43 \#4150)

[45] S. Watanabe and T. Yamada, On the uniqueness of solutions of stochastic differential equations. II, J. Math. Kyoto Univ. 11 (1971), 553-563. MR.0288876 (44 \#6071)

[46] T. Yamada, Sur une construction des solutions d'équations différentielles stochastiques dans le cas non-lipschitzien (French), Séminaire de Probabilités, XII (Univ. Strasbourg, Strasbourg, 1976/1977), Lecture Notes in Math., vol. 649, Springer, Berlin, 1978, pp. 114-131. MR.520000 (80k:60076)

[47] W. Zhou, The quasiderivative method for derivative estimates of solutions to degenerate elliptic equations, Stochastic Process. Appl. 123 (2013), 3064-3099, DOI 10.1016/j.spa.2013.03.021. MR.3062437

Department of Mathematics, Rutgers, The State University of New Jersey, 110 FrelINGHUYSEn RoAd, PiscataWAy, NeW Jersey 08854-8019

E-mail address: feehan@math.rutgers.edu

Department of Mathematics, University of Pennsylvania, 209 South 33rd Street, Philadelphia, Pennsylvania 19104-6395

E-mail address: cpop@math.upenn.edu 